\title{
DEMONSTRATED RESOLUTION ENHANCEMENT CAPABILITY OF A STRIPMAP HOLOGRAPHIC APERTURE LADAR SYSTEM
}

\author{
Thesis \\ Submitted to \\ The School of Engineering of the \\ UNIVERSITY OF DAYTON \\ In Partial Fulfillment of the Requirements for \\ The Degree \\ Master of Science in Electro-Optics
}

By

Samuel Martin Venable III

Dayton, Ohio

May, 2012

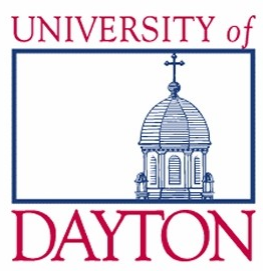




\section{DEMONSTRATED RESOLUTION ENHANCEMENT CAPABILITY OF A STRIPMAP HOLOGRAPHIC APERTURE LADAR SYSTEM}

Name: Venable, Samuel Martin

APPROVED BY:

Bradley D. Duncan, Ph.D.

Professor, Electro-Optics Program

Associate Dean, Graduate School

University of Dayton

Committee Chairman
Joseph W. Haus, Ph.D.

Professor and Director

Electro-Optics Program

University of Dayton

Committee Member

Matthew P. Dierking, Ph.D.

Principal Scientist

EO Combat ID Branch

Sensors Directorate

Air Force Research Labs

Committee Member

John G. Weber, Ph.D.

Associate Dean

School of Engineering

Tony E. Saliba, Ph.D. Dean, School of Engineering \&Wilke Distinguished Professor 


\section{ABSTRACT \\ DEMONSTRATED RESOLUTION ENHANCEMENT CAPABILITY OF A STRIPMAP HOLOGRAPHIC APERTURE LADAR SYSTEM}

Name: Venable, Samuel Martin

University of Dayton

Advisor: Dr. Bradley D. Duncan

Holographic aperture ladar (HAL) is a variant of synthetic aperture ladar (SAL). The two processes are related in that they both seek to increase cross-range (i.e., the direction of the receiver translation) image resolution through the synthesis of a large effective aperture - which is in turn achieved via the translation of a receiver aperture and the subsequent coherent phasing and correlation of multiple received signals. However, while SAL imaging incorporates a translating point detector, HAL takes advantage of twodimensional translating sensor arrays. For the research presented in this article, a side looking Stripmap HAL geometry was used to sequentially illuminate a set of Ronchi ruling targets. Prior to this, theoretical calculations were performed to determine the baseline, single sub-aperture resolution of our experimental, laboratory based system. Theoretical calculations were also performed to determine the ideal modulation transfer function (MTF) and expected cross-range HAL image sharpening ratio corresponding to the geometry of our apparatus. To verify our expectations, we first sequentially captured 
an over-sampled collection of pupil plane field segments for each Ronchi ruling. A HAL processing algorithm was then employed to phase correct and re-position the field segments after which they were properly aligned through a speckle field registration process. Relative piston and tilt phase errors were then removed prior to final synthetic image formation. By then taking the Fourier transform of the synthetic image intensity and examining the fundamental spatial frequency content, we were able to produce experimental modulation transfer function curves which we could then compare to our theoretical expectations. Our results show that we are able to achieve nearly diffraction limited results for image sharpening ratios as high as 6.43 . 


\section{ACKNOWLEDGEMENTS}

For providing the opportunity, laboratory space and equipment to complete the research presented in this thesis, I would like to thank Bryan Eurart, Chief of the ElectroOptic Combat Identification Technology branch of the Air Force Research Lab, AFRL/RYMM. Also for their guidance and encouragement, I am indebted to Dr. Bradley Duncan, my thesis advisor, and Dr. Matt Dierking, AFRL/RYMM technical advisor. For their invaluable advice in the lab, I would like to thank Dr. Dave Rabb, Jason Stafford and Doug Jameson. Finally, special thanks to my wife, Amanda, for her constant support and for putting up with the many late nights.

This effort was supported in part by the U.S. Air Force through contract number FA865006-02-1081, and the University of Dayton Ladar and Optical Communications Institute (LOCI). The views expressed in this article are those of the authors and do not reflect on the official policy of the Air Force, Department of Defense or the U.S. Government. 


\section{TABLE OF CONTENTS}

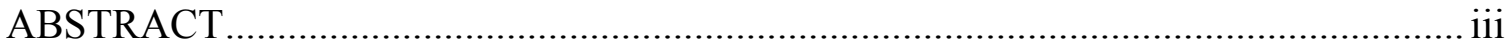

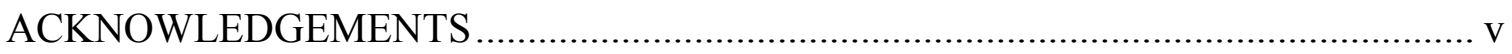

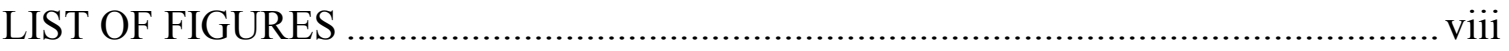

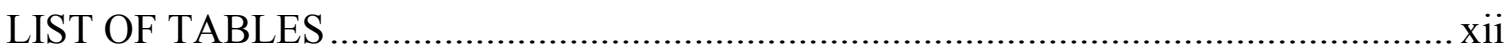

CHAPTER 1 INTRODUCTION ........................................................................ 1

$1.1 \quad$ Historical Background.............................................................................. 1

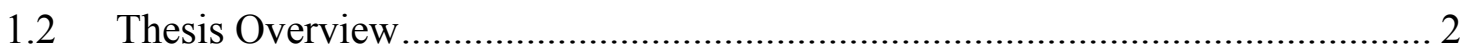

CHAPTER 2 HOLOGRAPHIC APERTURE LADAR ……………………………..... 5

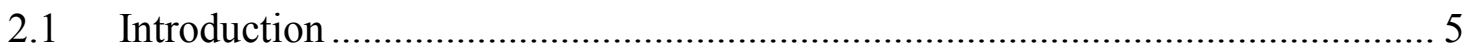

2.2 The Stripmap HAL Transformation ............................................................. 9

2.2.1 Off-Axis Point Target Special Case _............................................................ 14

2.2.2 Longitudinal Cross-Range Stripmap HAL Image Resolution ..................... 16

CHAPTER 3 PRELIMINARY CONSIDERATIONS ………………………........... 22

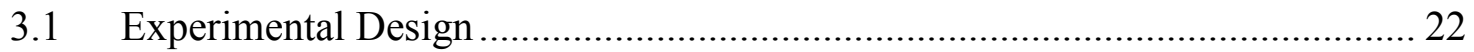

3.2 The Image Sharpening Ratio and Spatial Bandwidth ....................................... 26

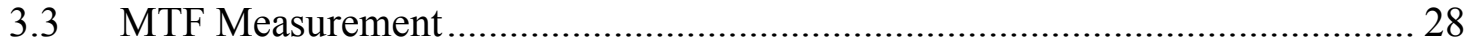

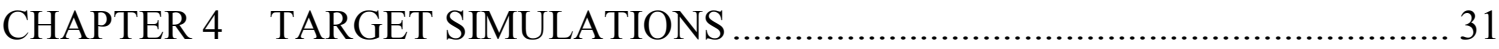

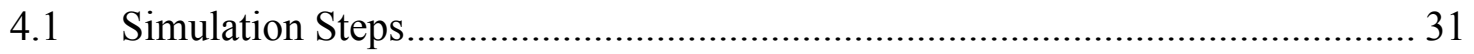




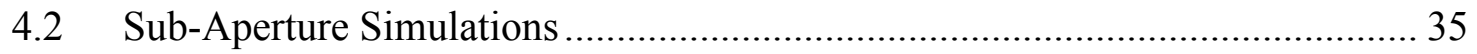

4.3 High Resolution Synthetic Image Simulations ........................................... 38

CHAPTER 5 DATA COLLECTION AND PROCESSING ...................................... 42

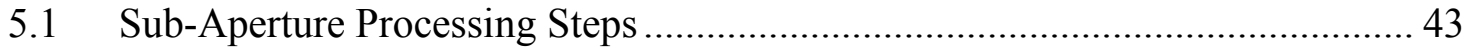

5.2 HAL Processing Steps.......................................................................... 49

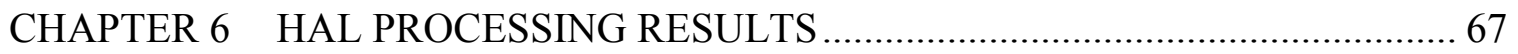

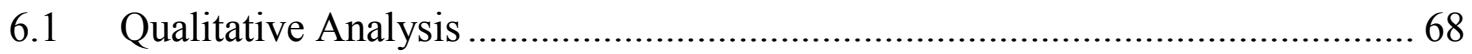

6.2 Quantitative Analysis ........................................................................... 74

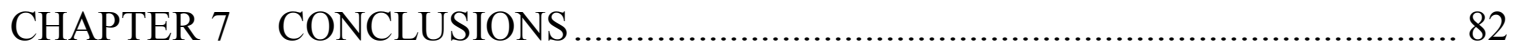

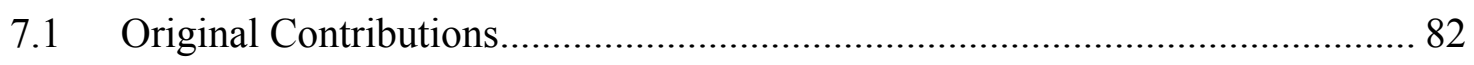

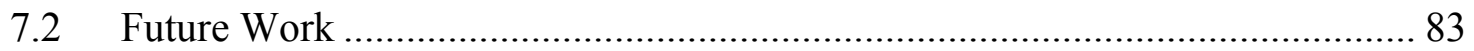

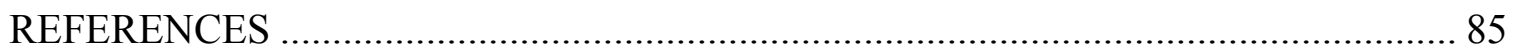

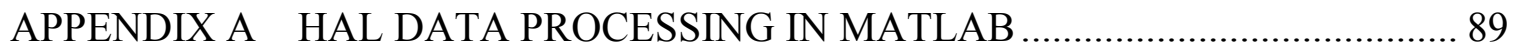

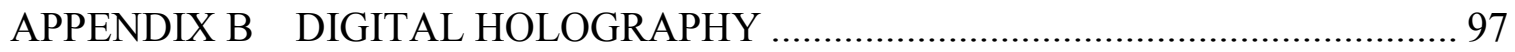

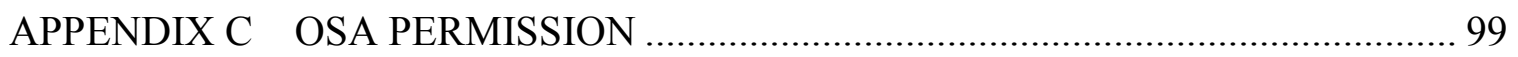




\section{LIST OF FIGURES}

Figure 1: Notional depiction of a Holographic Aperture Ladar system. The imaging sensor is assumed to be moving in the direction of flight of an airborne platform. For clarity, the transmitter and the receiver master oscillator are not shown.......................... 7

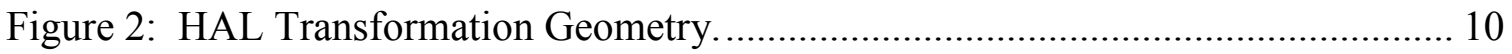

Figure 3: Uncorrected and corrected point object phase segments. Here the stripmap HAL transformation has been applied to a phase only field segment resulting from an offaxis point target. Both the transmitter and the receiver aperture are also off-axis in this bistatic TX/RX example. 15

Figure 4: Example of the stripmap HAL transformation applied to three sequentially collected phase only field segments resulting from an off-axis point target. In this case the RX aperture is effectively tangent to itself during each subsequent TX/RX cycle.

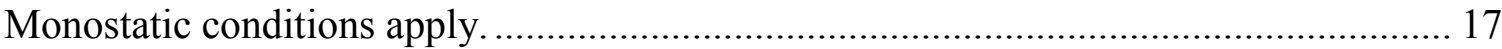

Figure 5: Example of the stripmap HAL transformation applied to five sequentially collected phase only segments resulting from an off-axis point target. In this case the RX aperture effectively overlaps itself by half its diameter during each subsequent TX/RX cycle. Monostatic conditions apply.... 18

Figure 6: Diagram of our experimental setup. 23

Figure 7: Lab setup containing the target object, $(0.25 \mathrm{cyc} / \mathrm{mm}$ as shown $)$ translation stage and local oscillator unit.

Figure 8: An idealized image intensity cross section for a 50\% duty cycle Ronchi ruling. The mean intensity is A, the peak-to-peak variation is $2 \mathrm{~B}$ and the spatial period is WR. 28

Figure 9: Simulation processing steps. 32

Figure 10: Example of a $0.25 \mathrm{cyc} / \mathrm{mm}$ target object that was created. 32

Figure 11: Final image of a $0.25 \mathrm{cyc} / \mathrm{mm}$ target used in the construction of the subaperture MTF. 34

Figure 12: Example of a cross section plot for $0.25 \mathrm{cyc} / \mathrm{mm}$ target. 35 
Figure 13: Final image of a $0.50 \mathrm{cyc} / \mathrm{mm}$ target used in the construction of the subaperture MTF.

Figure 14: Final image of a $0.75 \mathrm{cyc} / \mathrm{mm}$ target used in the construction of the subaperture MTF. 36

Figure 15: Final image of a $1.00 \mathrm{cyc} / \mathrm{mm}$ target used in the construction of the subaperture MTF.

Figure 16: Final image of a $1.25 \mathrm{cyc} / \mathrm{mm}$ target used in the construction of the subaperture MTF 37

Figure 17: Simulated sub-aperture image MTF. 38

Figure 18: Final image of a $1.00 \mathrm{cyc} / \mathrm{mm}$ target used in the construction of the synthetic image MTF. 39

Figure 19: Final image of a $2.00 \mathrm{cyc} / \mathrm{mm}$ target used in the construction of the synthetic image MTF 39

Figure 20: Final image of a $4.00 \mathrm{cyc} / \mathrm{mm}$ target used in the construction of the synthetic image MTF. 40

Figure 21: Final image of a $6.00 \mathrm{cyc} / \mathrm{mm}$ target used in the construction of the synthetic image MTF. 40

Figure 22: Final image of an $8.00 \mathrm{cyc} / \mathrm{mm}$ target used in the construction of the synthetic image MTF. 41

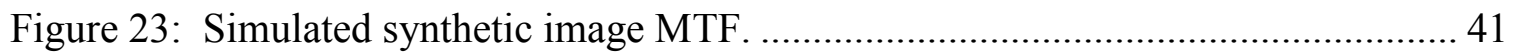

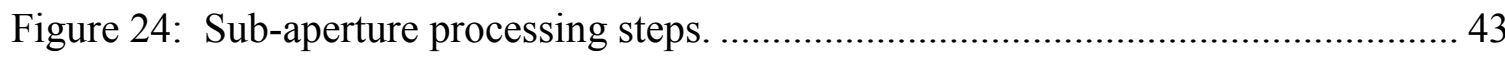

Figure 25: Image plane target information (a) and a crop of the target information....... 44

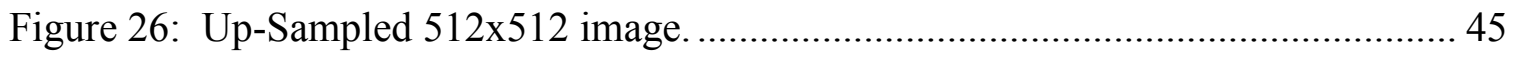

Figure 27: Shows the effects of proper sampling. (a) The spatial frequency content of an under-sampled target image. (b) Spatial frequency content of a properly sampled target image.. . 46

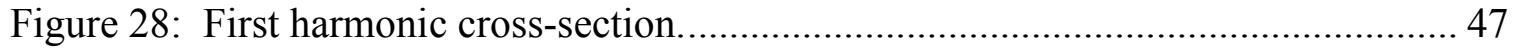

Figure 29: Cross section showing positive and negative first harmonic peaks. ............. 48

Figure 30: The HAL data processing method......................................................... 50 
Figure 31: An example off-axis hologram of our $0.25 \mathrm{cyc} / \mathrm{mm}$ target containing a digitally cropped array of $256 \times 256$ pixels. The somewhat discernible diagonal structure of this image is due to the tilted LO beam..... 51

Figure 32: Modulus of the Fourier transform of the hologram shown in Figure 31. The 256x256 array contains two focused images of the target and the central LO autocorrelation term. 52

Figure 33: (a) The cropped and zero-padded image term of Figure 5 is (b) inverse Fourier transformed back to the pupil plane. Only the moduli of the complex fields are shown in both cases. 53

Figure 34: Modulus of the composite, effective pupil plane array (a) before amplitude equalization and (b) after amplitude equalization. 57

Figure 35: Fourier transform of the synthetic pupil resulting in an image plane segment. 57

Figure 36: The final HAL processed synthetic image. The image was up-sampled to $512 \times 512$ by cropping out the target area from Figure 35 , IFFT to the pupil plane, zeropadding to $512 \times 512$ and FFT back to the image plane. 62

Figure 37: Side by side visual comparison of a $0.25 \mathrm{cyc} / \mathrm{mm}$ (a) sub-aperture image, and (b) a HAL processed synthetic image. 63

Figure 38: Side by side visual comparison of, (a) a $1 \mathrm{cyc} / \mathrm{mm}$ sub-aperture image, and (b) HAL processed synthetic image.

Figure 39: The spatial frequency content of the HAL processed synthetic image. The final synthetic image was converted to intensity, zero-padded to 4096x4096 and Fourier transformed to show the spatial frequency harmonics. 65

Figure 40: Side by side visual comparison of a, (a) $0.25 \mathrm{cyc} / \mathrm{mm}$ sub-aperture image and (b) HAL processed synthetic image.

Figure 41: Side by side visual comparison of a, (a) $0.50 \mathrm{cyc} / \mathrm{mm}$ sub-aperture image and (b) HAL processed synthetic image.

Figure 42: Side by side visual comparison of a, (a) $0.75 \mathrm{cyc} / \mathrm{mm}$ sub-aperture image and (b) HAL processed synthetic image. 70

Figure 43: Side by side visual comparison of a, (a) $1 \mathrm{cyc} / \mathrm{mm}$ sub-aperture image and (b) HAL processed synthetic image. 70

Figure 44: Side by side visual comparison of a, (a) $1.25 \mathrm{cyc} / \mathrm{mm}$ sub-aperture image and (b) HAL processed synthetic image. 
Figure 45: Side by side visual comparison of a, (a) $2 \mathrm{cyc} / \mathrm{mm}$ sub-aperture image and (b) HAL processed synthetic image.

Figure 46: Side by side visual comparison of a, (a) $4 \mathrm{cyc} / \mathrm{mm}$ sub-aperture image and (b) HAL processed synthetic image and (c) a zoomed region of the HAL processed synthetic image. The vertical structure of the synthetic image is evident in the zoomed image. ... 72

Figure 47: Side by side visual comparison of a, (a) $6 \mathrm{cyc} / \mathrm{mm}$ sub-aperture image and (b) HAL processed synthetic image and (c) a zoomed region of the HAL processed synthetic image. The vertical structure of the synthetic image is evident in the zoomed image. .... 73

Figure 48: Side by side visual comparison of a, (a) $8 \mathrm{cyc} / \mathrm{mm}$ sub-aperture image and (b) HAL processed synthetic image and (c) a zoomed region of the HAL processed synthetic image. The vertical structure of the synthetic image is evident in the zoomed image. .... 74

Figure 49: Theoretical and experimental MTF functions for single sub-aperture imaging. 75

Figure 50: Theoretical and experimental MTF functions for synthetic images formed using 12 overlapping frames of fully HAL processed data. 76

Figure 51: Theoretical and experimental MTF functions for synthetic images formed using 8 overlapping frames of fully HAL processed data. More nearly diffraction limited performance is realized in exchange for reduced image sharpening. 78

Figure 52: Theoretical and experimental MTF functions for synthetic images formed using 5 overlapping frames of fully HAL processed data. More nearly diffraction limited performance is realized in exchange for reduced image sharpening. 80

Figure 53: Experimental sub-aperture and full HAL processed MTF displayed on the same plot to show the cut-off gain 81 


\section{LIST OF TABLES}

Table 1: Optimization data for the $6.00 \mathrm{cyc} / \mathrm{mm}$ target data set. The iterations are presented along with their respective optimization variable and $\mathrm{S}_{\min }$ value.

Table 2: Optimization data for the $1.00 \mathrm{cyc} / \mathrm{mm}$ target data set. The iterations are presented along with their respective optimization variable and $S_{\min }$ value................... 61

Table 3: HAL processed data for the multiple targets used in the construction of the HAL processed MTF. The values listed for each data set were obtained using the HAL processing algorithm in Figure 30 and have the $\pi / 2$ scaling factor applied to the data... 66

Table 4: Sub-aperture data for the multiple targets used in the construction of the subaperture processed MTF. The values listed for each data set were obtained using the steps found in Figure 24 and have the $\pi / 2$ scaling factor applied to the data. ................ 66

Table 5: A table constructed to show the metrics used to analyze the performance of the HAL processed MTF for $\mathrm{N}=12$ sub-shots. The system baseline values and results are shown as well as percent error between theoretical and actual measurements

Table 6: A table constructed to show the metrics used to analyze the performance of the HAL processed MTF for $\mathrm{N}=8$ sub-shots. The system baseline values and results are shown as well as percent error between theoretical and actual measurements. 79

Table 7: A table constructed to show the metrics used to analyze the performance of the HAL processed MTF for $\mathrm{N}=5$ sub-shots. The system baseline values and results are shown as well as percent error between theoretical and actual measurements 80 


\section{CHAPTER 1}

\section{INTRODUCTION}

Synthetic Aperture Radar (SAR) has been used to overcome limited resolution in long range remote sensing applications. This specific imaging technique uses a translating point detector and uses the platform motion of the point detector to collect multiple coherent sub-shots of data. The cross-range data that is collected will then serve to form the larger synthesized receiver aperture. This will ultimately lead to an increase in longitudinal, cross range resolution.

\subsection{Historical Background}

Synthetic Aperture Ladar (SAL) methods can be used to overcome traditional image resolution limits in long range remote sensing applications. This imaging technique employs a translating point detector and sensor platform motion to sequentially collect multiple coherent sub-shots of data in the longitudinal cross-range dimension (i.e., in the direction of sensor motion). Data collected in this fashion is then coherently phased to form a large, effective receiver aperture. After phase errors accumulated across the synthetic aperture are corrected, subsequent post-processing can ultimately lead to substantial increases in longitudinal cross-range image resolution [1]. 
Holographic Aperture Ladar (HAL) is another aperture synthesis technique akin to SAL. The techniques are similar in that each makes use of a translating sensor to build up a larger effective receiver aperture through the coherent processing of sequentially collected data. However, the HAL technique is dissimilar to SAL due to use of a two dimensional translating imaging sensor instead of a simple point detector. A key advantage of the HAL process is that it is capable of producing images of remote targets in two orthogonal cross-range dimensions - also known as angle-angle imaging [2]-[3]. In this article we will focus on demonstrating the resolution enhancement capabilities of a side-looking stripmap HAL system.

\subsection{Thesis Overview}

Our previous work first focused on the theoretical development of the Stripmap HAL transformation [2]. Subsequent numerical analyses demonstrated that accounting for motion induced phase migrations across a sequential collection of pupil plane field segments could in fact produce an effective aperture capable of yielding enhanced image resolution. However, these early results assumed the prior existence of multiple pupil field segments and instead focused on the mathematical descriptions of the stripmap HAL transformation [2].

The next step was to experimentally verify the Stripmap HAL transformation's ability to account for off-axis transmitter induced phase migrations, as well as verify the transform's ability to produce an increase in longitudinal cross-range image resolution through creation of an extended coherent pupil plane field [3]. While successful, initial experimentation was performed using a simple point target (i.e., a small, polished ball 
bearing) which lacked the intricate phase information of a more complex, extended target. To better demonstrate the Stripmap HAL transform's ability to enhance resolution and account for various phase errors accumulated during the data collection process, we have used extended, diffuse targets for the work described herein.

For the work presented in this article a collection of diffusely reflecting Ronchi ruling targets were chosen at a variety of spatial frequencies to allow sufficient sampling across the spatial frequency spectra of both a diffraction limited single sub-aperture, as well as a fully HAL processed effective aperture. As we will demonstrate, the use of multiple single frequency targets allows us to conveniently map out the modulation transfer function (MTF) of our system, thereby precisely demonstrating the resolution increases capable as a direct result of HAL processing.

Prior to experimentation, theoretical calculations were performed to determine the baseline, single sub-aperture resolution of our laboratory based system. Theoretical calculations were also performed to determine the ideal modulation transfer function (MTF) and expected cross-range HAL image sharpening ratio corresponding to the geometry of our apparatus. To verify our expectations, we first sequentially captured an over-sampled collection of pupil plane field segments for each Ronchi ruling. A HAL processing algorithm was then employed to phase correct and re-position the field segments, after which they were properly aligned through a speckle field registration process. Relative piston and tilt phase errors were then removed prior to final synthetic image formation. By then taking the Fourier transform of the synthetic image intensity and examining the fundamental spatial frequency content, we were able to produce experimental modulation transfer function curves which we then compared to theoretical 
expectations. Our results show that we are able to achieve nearly diffraction limited results for image sharpening ratios as high as 6.43 . 


\section{CHAPTER 2}

\section{HOLOGRAPHIC APERTURE LADAR}

In this chapter we present the basics of HAL theory. The following text was originally presented in Applied Optics, Volume 48, pages 1168-1177. A portion of the text is presented, in particular the generation and discussion of the Stripmap HAL transformation. [2] The selection in this chapter is reprinted with permission from the Optical Society of America. ${ }^{\mathrm{i}}$

\subsection{Introduction}

Traditional synthetic aperture radar (SAR) and ladar (SAL) imaging methods rely upon the availability of temporally coherent return data and the use of matched filter pulse compression techniques in order to achieve high resolution. While for a single pulse good range resolution is obtained via temporal pulse compression, cross-range resolution is often poor and diminishes with range as the transmitted pulse continues to diffract. High resolution in the longitudinal cross-range dimension (i.e., in the direction of flight in the case of an airborne SAR/SAL sensor) is instead achieved by using a single antenna/detector acting in essence as a point receiver to collect spatially coherent data at multiple uniformly spaced locations along an extended path. By combining the cross- 
range data in post-detection signal processing, an effectively larger cross-range receiver aperture can be synthesized, thereby resulting in increased cross-range resolution. The result of this extensive post-processing is a two dimensional (i.e., range and longitudinal cross-range) map, or image, of object scene reflectivity at the interrogation wavelength [4]-[13].

Holographic Aperture Ladar (HAL) is a variant of SAL in that it is a method of increasing the ability of an optical imaging system to resolve fine cross-range scene detail by synthesizing a large effective aperture through the motion of a smaller receiver, and through the subsequent proper phasing and correlation of the detected signals in postprocessing. Unlike traditional SAR and SAL, however, HAL will make use of a twodimensional translating sensor array, not simply a translating point detector.

The general concept of holographic aperture imaging is shown notionally in Figure 1. In this figure we show a single translating sensor array being used to sequentially capture multiple longitudinal cross-range segments of the incoming complex (i.e., amplitude and phase) pupil plane field. Although direct pupil plane measurements are possible [7][14][15], due in part to the benefits of aperture gain in an imaging configuration we assume in Figure 1 that image plane measurements are made. More specifically, the complex field at the image plane can be determined by capturing an image of a coherently illuminated scene at each sub-aperture location. By interfering each scene sub-image with a known optical master oscillator (MO), not shown in Figure 1, the image plane amplitude and phase can be recorded and extracted in post processing, for example through use of phase stepping interferometry [7]. The pupil plane complex field segments associated with each sub-aperture capture location are then related to the image 
plane measurements through an inverse Fourier transform operation, after which the subaperture fields are placed in a single synthetic pupil plane matrix according to one of the HAL transformations to be developed in the following sections. A higher resolution image is then synthesized digitally by first applying a virtual lens to the synthetic pupil plane field and propagating to a virtual focal plane detector, the image plane field being formed via discrete Fourier transform. The synthetic image is then the squared modulus of the result.

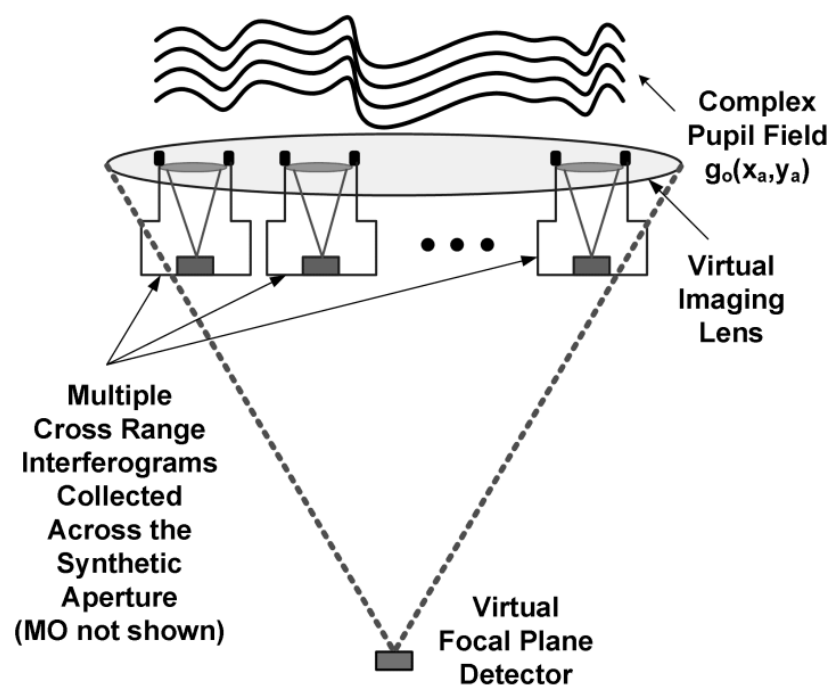

Figure 1: Notional depiction of a Holographic Aperture Ladar system. The imaging sensor is assumed to be moving in the direction of flight of an airborne platform. For clarity, the transmitter and the receiver master oscillator are not shown. ${ }^{1}$

The focus of this article will be on how the individual pupil plane field segments, can be properly stitched together to synthetically form a complex pupil plane field with a larger spatial extent. The challenge in this process will be in accounting for the practical fact that both the receiver aperture and the transmitter will both be in synchronous motion

\footnotetext{
${ }^{1}$ Reprinted with permission from Applied Optics 48, 1168-1174, (C) Optical Society of America.
} 
(i.e., with equal velocities) in real-world airborne HAL sensor applications. To our knowledge this issue has never before been fully addressed [7][15].

We have come to call this area of research Holographic Aperture Ladar because we are working in the optical regime where only time averaged intensity records are currently possible. Most likely then, by using 2D interferograms recorded in either the pupil or image planes at each sub-aperture collection site, we wish to reconstruct (digitally) and properly stitch together the complex pupil field segments found for each sub-aperture [7][14][15][16]. However, while the notion of reconstructing complex fields from interference records is generally reminiscent of holography we do not claim that traditional holographic methods for determining the complex pupil plane field segments must necessarily be employed, or are that they are even required. Nevertheless the idea of holographic aperture ladar seems to capture and call to mind the essential elements of our technique.

In this article we will assume that the complex pupil plane field segments are available to us without regard to how they were obtained. We will instead focus primarily on developing the transformations that will allow complex pupil plane field segments to be stitched together coherently. We will also present some intriguing consequences related to two dimensional high resolution image formation. Unlike in traditional SAR/SAL, however, the images we will discuss will be formed in the two orthogonal cross-range dimensions, sometimes known as angle-angle imaging [16]. That is, our image coordinates will be in the longitudinal cross-range dimension, parallel to the direction of flight, and the transverse cross-range dimension, perpendicular to the direction of flight, assuming an airborne sensor platform. In our work here we will also 
consider only a single translating sub-aperture. As a result, the transverse cross-range resolution will be dictated by the sub-aperture pupil dimensions alone. On the other hand, the longitudinal cross-range resolution will be enhanced, as in the case of traditional SAR/SAL, by virtue of the synthetic aperture created through longitudinal motion of the sub-aperture sensor array.

While target range and scene depth information will be encoded within the synthetic pupil plane field, as in conventional holography, we will demonstrate that in practical applications the HAL transformation process is relatively insensitive to scene depth if a good estimate of nominal scene range is available. Nevertheless, we envision that this information may be extracted, allowing three dimensional (i.e., angle-anglerange) imaging, if additional variability is introduced into the system; e.g., via temporal waveform shaping and conventional pulse compression, or multiple wavelength sensing schemes [11][17][18][19]. Extracting scene depth information will, however, be a topic for further research and discussion at a later time.

\subsection{The Stripmap HAL Transformation}

The central issue to be overcome in properly phasing together multiple pupil plane field segments captured across the synthetic aperture is the fact that both the transmitter (TX) and receiver (RX) aperture will be in synchronous, ideally linear, motion. The transformations we will develop in this and the following section will effectively allow us to relocate the TX to a fixed position at the center of the receiver plane coordinate system for all shots taken across the synthetic aperture. 
In this section we will develop the side looking stripmap HAL transformation. In this scenario the TX beam is always directed orthogonal to the direction of sensor motion in such a way that the illumination beam sweeps linearly across the scene of interest. In all cases we will assume that the target is fully (i.e., flood) illuminated by a single, though moving, coherent TX beam for all shots across the synthetic aperture, and that the scene remains unchanged over multiple looks. As ladar synthetic apertures are typically on the order of 1-2 m, while the range $\mathrm{R}_{\mathrm{o}}$ to target is on the order of 10 's of $\mathrm{km}$, this second assumption is quite reasonable. For the same reasons we will also assume the target scene to be nominally planar. Moreover, while the TX will most likely be an untruncated, collimated (at the TX aperture) Gaussian beam we will not address the effects of nonuniform scene illumination. We will only consider the transmitter's spherical phase front, with radius of curvature $\mathrm{R}_{\mathrm{o}}$ (assuming the target range is much larger than TX beam's Rayleigh range), as it illuminates the scene. We will also ignore all atmospheric effects.

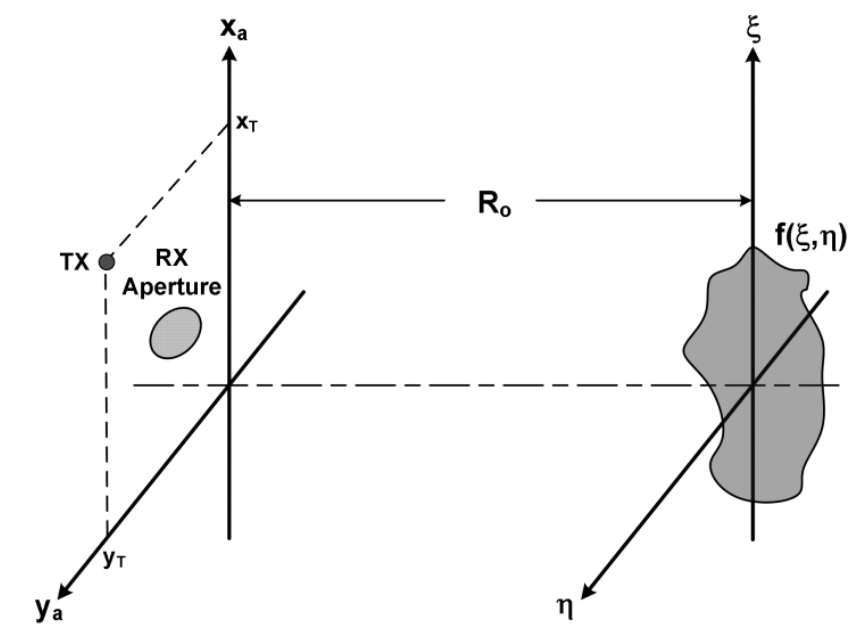

Figure 2: HAL Transformation Geometry. ${ }^{2}$

\footnotetext{
${ }^{2}$ Reprinted with permission from Applied Optics 48, 1168-1174, (C) Optical Society of America.
} 
The geometry of the problem is depicted in Figure 2. Shown in this figure is a single TX located at $\left(x_{T}, y_{T}\right)$ in the aperture plane coordinate system. The transmitter illuminates the target $f(\xi, \eta)$ at range $R_{o}$, and the returning complex field is collected by a single off-axis RX aperture, the location of which will not affect the HAL transformation. Notice that the receiver plane coordinates are given by variables $\left(x_{a}, y_{a}\right)$, while the target plane coordinate variables are $(\xi, \eta)$.

The field $g_{s m}\left(x_{a}, y_{a}\right)$, where the $s m$ subscript indicates the stripmap case, in the plane of the receiver aperture is found through scalar diffraction theory according to the relationship

$$
g_{s m}=C\left\{f(\xi, \eta) \exp \left(j \frac{\pi}{\lambda R_{0}}\left[\left(\xi-x_{T}\right)^{2}+\left(\eta-y_{T}\right)^{2}\right]\right) \otimes h_{R_{0}}(\xi, \eta)\right\},
$$

where $C$ is a complex constant, $\otimes$ represents convolution, $h_{R o}$ is the free-space impulse response given by

$$
h_{R_{o}}=\exp \left(j \frac{\pi}{\lambda R_{o}}\left(\xi^{2}+\eta^{2}\right)\right)
$$

and where off-axis spherical wave illumination of the target has been assumed. Assuming for now that $f(\xi, \eta)$ in Eq. (1) is a separable function, we can proceed with the details of the derivation in only one dimension. Expanding and simplifying Eq. (1) in one dimension then yields

$$
g_{s m}\left(x_{a}\right)=C \int_{-\infty}^{\infty} f(\xi) \exp \left(j \frac{\pi}{\lambda R_{0}}\left(\xi-x_{T}\right)^{2}\right) \exp \left(j \frac{\pi}{\lambda R_{0}}\left(x_{a}-\xi\right)^{2}\right) d \xi
$$




$$
\begin{gathered}
=C \exp \left(j \frac{\pi}{\lambda R_{o}} x_{a}^{2}\right) \exp \left(j \frac{\pi}{\lambda R_{o}} x_{T}^{2}\right) \int_{-\infty}^{\infty} f(\xi) \exp \left(j \frac{2 \pi}{\lambda R_{o}} \xi^{2}\right) \exp (-j p \xi) d \xi \\
=C \exp \left(j \frac{\pi}{\lambda R_{o}} x_{a}^{2}\right) \exp \left(j \frac{\pi}{\lambda R_{o}} x_{T}^{2}\right) F\left(x_{a}+x_{T}\right),
\end{gathered}
$$

where Eq. (2) is recognized as a Fourier transform integral with radian spatial frequency variable $p$ given by

$$
p=\frac{2 \pi}{\lambda R_{o}}\left(x_{a}+x_{T}\right)[\mathrm{rad} / \mathrm{m}]
$$

and where $F$ in Eq. (3) is the Fourier transform of $f(\xi) \exp \left(j 2 \pi \xi^{2} / \lambda R_{o}\right)$. Ideally, however, our transmitter would be located at $x_{T}=0$, leading to a received field $g_{o}\left(x_{a}, y_{a}\right)$ given in one dimension as

$$
g_{o}\left(x_{a}\right)=C \exp \left(j \frac{\pi}{\lambda R_{o}} x_{a}^{2}\right) F\left(x_{a}\right)
$$

In order to then express $g_{o}$ in terms of known or measured quantities $g_{s m}\left(x_{a}\right)$ and $x_{T}$ we first subtract $x_{T}$ from $x_{a}$ in Eq. (3) and rearrange to yield

$$
g_{s m}\left(x_{a}-x_{T}\right) \exp \left(-j \frac{\pi}{\lambda R_{0}} x_{T}^{2}\right)=C \exp \left(j \frac{\pi}{\lambda R_{0}}\left(x_{a}-x_{T}\right)^{2}\right) F\left(x_{a}\right) .
$$

Next we expand the quadratic phase term on the right hand side of Eq. (6) and simplify to find

$$
g_{s m}\left(x_{a}-x_{T}\right) \exp \left(-j \frac{2 \pi}{\lambda R_{0}} x_{T}\left(x_{T}-x_{a}\right)\right)=C \exp \left(j \frac{\pi}{\lambda R_{0}} x_{a}^{2}\right) F\left(x_{a}\right)=g_{0}\left(x_{a}\right)
$$

One further rearrangement of Eq. (7) then yields, in two dimensions, the desired stripmap HAL transformation expressed as 


$$
g_{0}\left(x_{a}+x_{T}, y_{a}+y_{T}\right)=g_{s m}\left(x_{a}, y_{a}\right) \exp \left(j \frac{2 \pi}{\lambda R_{0}}\left[x_{a} x_{T}+y_{a} y_{T}\right]\right) .
$$

We see from Eq. (8) that the stripmap HAL transformation is quite simple and involves only a linear phase correction applied to the detected pupil plane field, according to the location of the transmitter at the instant the field was received, and a repositioning of the phase corrected field segment. Moreover, it is easy to verify that had the derivation of Eq. (8) been performed in two dimensions we would have obtained the same result even if we had not assumed $f(\xi, \eta)$ was separable.

Notice that the stripmap HAL transformation is complete and rigorous, and involves no approximations or assumptions other than those required for scalar diffraction theory to apply [20]. In particular, since no attempt was made to ignore or eliminate the various quadratic phase terms in Eq. (2), the HAL transformation expressed in Eq. (8) is valid for targets both in the Fresnel and Fraunhoffer diffraction zones, or at least at any range $R_{o}$ that is much greater than the Rayleigh range, if the TX beam is Gaussian.

In addition, recall that in Figure 2 the center of the RX aperture was not specified. Implicit then in Eq. (8) is the assumption that the received field $g_{s m}\left(x_{a}, y_{a}\right)$ will have nonzero value only over the physical extent of the receiver aperture. For example, if during one TX/RX event the receiver aperture extends from $x_{a}=0.2 \mathrm{~m}$ to $x_{a}=0.3 \mathrm{~m}$, in the $\mathrm{x}-$ dimension, while the transmitter is located at $x_{T}=0.25 \mathrm{~m}$ (i.e., monostatic conditions), then after the required linear phase correction, the repositioned idealized field segment would extend from $x_{a}=0.45 \mathrm{~m}$ to $x_{a}=0.55 \mathrm{~m}$. 


\subsubsection{Off-Axis Point Target Special Case}

To demonstrate the application of the stripmap HAL transformation, consider now the one-dimensional special case of a target consisting of a single off-axis point scatterer. Only a single TX/RX cycle will be examined here, while the effects of collecting and correcting multiple sequentially collected field segments will be examined more fully in the Section 2.2.2.

Inserting $f(\xi)=\delta\left(\xi-\xi_{p}\right)$, where $\delta$ indicates the Dirac delta function, into Eq. (2) yields

$$
g_{p-s m}\left(x_{a}\right)=C \exp \left(j \frac{\pi}{\lambda R_{0}}\left(x_{a}^{2}+x_{T}^{2}\right)\right) \int_{-\infty}^{\infty} \delta\left(\xi-\xi_{p}\right) \exp \left(j \frac{2 \pi}{\lambda R_{0}} \xi^{2}\right) \exp (-j p \xi) d \xi
$$

where the $p$ subscript indicates the field received for the point target case. After applying the sifting property of the Dirac delta function to Eq. (9), and then substituting Eq. (4) into the result and simplifying, we find the following phase only result

$$
g_{p-s m}\left(x_{a}\right)=C \exp \left(j \frac{2 \pi}{\lambda R_{0}}\left(\frac{x_{a}^{2}+x_{T}^{2}}{2}+\xi_{p}^{2}-\left(x_{a}+x_{T}\right) \xi_{p}\right)\right) .
$$

Similarly, by setting $x_{T}=0$, we obtain the ideal field given by

$$
g_{p 0}\left(x_{a}\right)=C \exp \left(j \frac{2 \pi}{\lambda R_{0}}\left(\frac{x_{a}^{2}}{2}+\xi_{p}\left(\xi_{p}-x_{a}\right)\right)\right) .
$$

(Note that the result of Eq. (10) can be independently verified using simple geometry to calculate the round trip propagation distance $D_{R T}$ from the transmitter, to the target, and back to an arbitrary point in the RX aperture plane. The field $g_{p \text {-sm }}\left(x_{a}\right)$ will then be $\exp \left(j 2 \pi D_{R T} / \lambda\right)$. After simplification the same result expressed in Eq. (10) will be obtained.) 
As a specific example, we set $\xi_{p}=0.25 \mathrm{~m}$ and assume the transmitter is located at $x_{T}=-0.25 \mathrm{~m}$. We will also assume bistatic ladar conditions and let the receiver aperture extend from $x_{a}=0.25 \mathrm{~m}$ to $x_{a}=0.45 \mathrm{~m}$. Furthermore, we will assume that the range to target is $R_{o}=30 \mathrm{~km}$, and that the TX wavelength is $\lambda=1.5 \mu \mathrm{m}$. The results are shown in Figure 3.

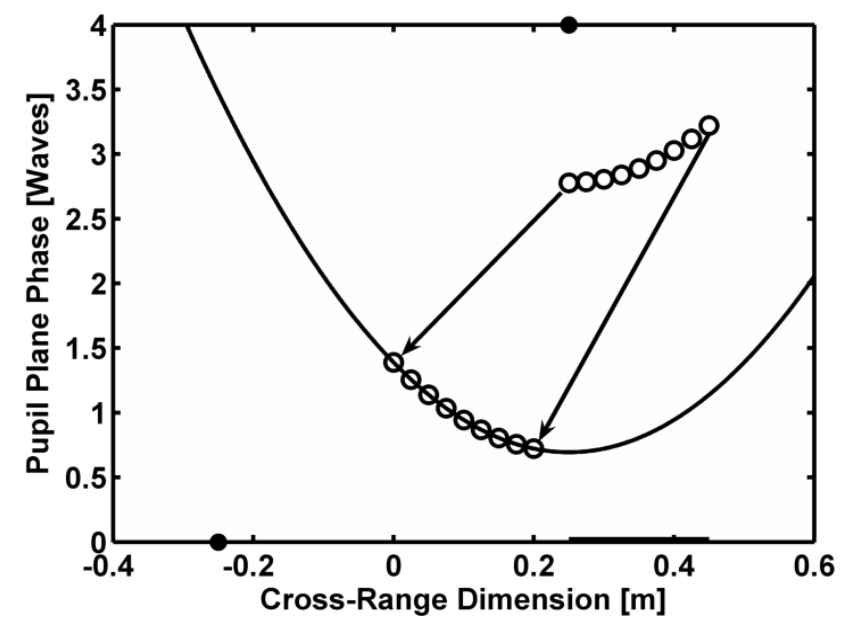

Figure 3: Uncorrected and corrected point object phase segments. Here the stripmap HAL transformation has been applied to a phase only field segment resulting from an off-axis point target. Both the transmitter and the receiver aperture are also off-axis in this bistatic TX/RX example. ${ }^{3}$

Along the horizontal axis of this figure the bold line segment shows the location of the $\mathrm{RX}$ aperture while the black dot indicates the location of the transmitter. Similarly, the black dot on the upper border of the figure indicates the longitudinal cross-range location of the point target (assuming, of course, that the target is actually at the proper range $R_{o}$ ). The raw phase segment in the upper right hand portion of the figure, indicated by the circular data points, was then calculated by substituting the appropriate values into Eq. (10). The ideal phase, shown by the solid black curve, was determined by plotting Eq.

\footnotetext{
${ }^{3}$ Reprinted with permission from Applied Optics 48, 1168-1174, (C) Optical Society of America.
} 
(11) over an extended range of longitudinal cross-range values, and the corrected phase segment, also indicated by circular data points, shows the result of applying the stripmap HAL transformation to the raw phase values. We see very clearly from Figure 3 that the stripmap HAL transformation precisely corrects for the effects due to an off-center TX.

\subsubsection{Longitudinal Cross-Range Stripmap HAL Image Resolution}

Consider now the case wherein both a single translating RX aperture and the TX are in motion. As in the previous example we shall examine this problem in only one dimension and will begin by assuming a single point target at a range of $R_{o}=30 \mathrm{~km}$, with a longitudinal cross-range location of $\xi_{p}=0.25 \mathrm{~m}$. We will also assume the TX wavelength is $\lambda=1.5 \mu \mathrm{m}$. In the following two examples, though, we will assume we have a receiver aperture with diameter $D_{a p}=0.4 \mathrm{~m}$, and will also assume monostatic conditions (i.e., the TX beam is centered upon and exits through the RX aperture). Moreover, in keeping with conventional SAR notation, we will assume that the synthetic aperture diameter $D_{S A R}$ is defined by the motion of the transmitter [1]. In particular, in the two examples to follow we will assume $D_{S A R}=0.8 m$, extending from $x_{a}=-0.4 \mathrm{~m}$ to $x_{a}=$ $0.4 \mathrm{~m}$.

Figure 4 demonstrates the results of correcting three sequentially collected phase segments captured across the synthetic aperture. In this case each shot is spaced by $0.4 \mathrm{~m}$ from its nearest neighbors, effectively forcing the real RX aperture to be tangent to itself during consecutive TX/RX cycles. As in Figure 3, the black dots along the horizontal axis of Figure 4 indicate the location of the transmitter during each shot, while the black dot on the upper border of the figure indicates the longitudinal cross-range location of the point target. Each of the raw phase segments were calculated by substituting appropriate 
values into Eq. (10). The ideal phase, shown by the solid black curve, was again determined by plotting Eq. (11) over an extended range of longitudinal cross-range values, while each of the corrected phase segments lying along the ideal phase curve represent the results of applying the stripmap HAL transformation to the raw phase values shown with corresponding data point markers.

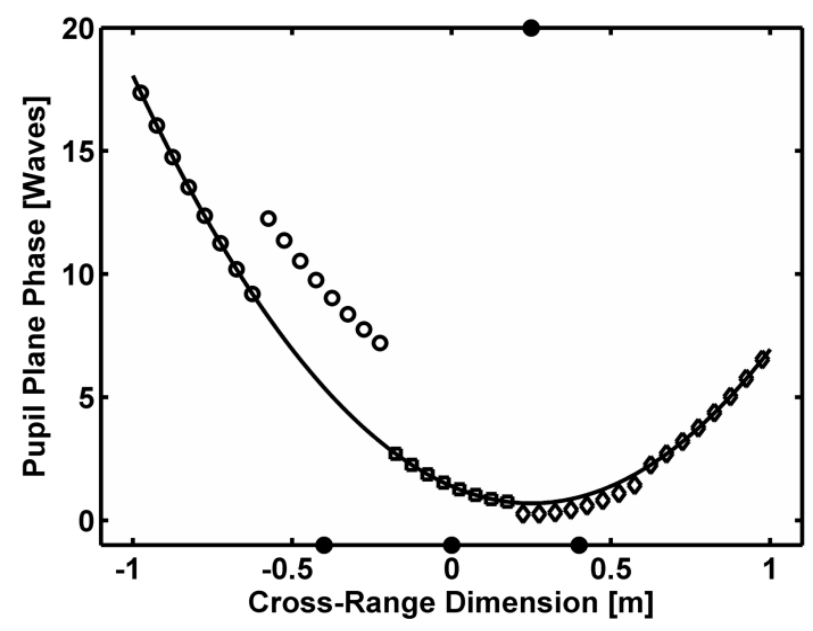

Figure 4: Example of the stripmap HAL transformation applied to three sequentially collected phase only field segments resulting from an off-axis point target. In this case the $\mathrm{RX}$ aperture is effectively tangent to itself during each subsequent TX/RX cycle. Monostatic conditions apply. ${ }^{4}$

Notice in Figure 4 that the phase segment shown with square data point markers, captured when $x_{T}=0$, needs no correction. This is, of course, true in general for any pupil field captured when the transmitter is centered on the RX aperture plane, since if both $x_{T}$ and $y_{T}$ equal zero Eq. (3) and (5) are identical. Notice also from Figure 4 that while the translating RX aperture effectively covers the entire synthetic aperture with no gaps, the corrected synthetic field has uniformly spaced gaps each of which is $D_{a p}$ wide. These gaps in the synthetic field would clearly have adverse effects on image resolution and

\footnotetext{
${ }^{4}$ Reprinted with permission from Applied Optics 48, 1168-1174, (C) Optical Society of America.
} 
would introduce periodic grating-like diffraction artifacts in any image created from the synthetic field as the number of cross-range shots increases.

In order to avoid this effect and optimally increase the resolution of HAL images it is clear that additional raw phase segments must be collected, each of which must be spaced by no more than $D_{a p} / 2$. To demonstrate this requirement consider Figure 5 . This figure was created by using the same parameters and conditions used to generate Figure 4. However, for Figure 5 five sequential TX/RX cycles were assumed, each of which was spaced by $D_{a p} / 2=0.2 \mathrm{~m}$. Again, the corrected phase segments lying along the ideal phase curve show the results of applying the stripmap HAL transform to the raw phase values shown with corresponding data point markers. We clearly see that the synthetically reconstructed pupil plane field is now continuous.

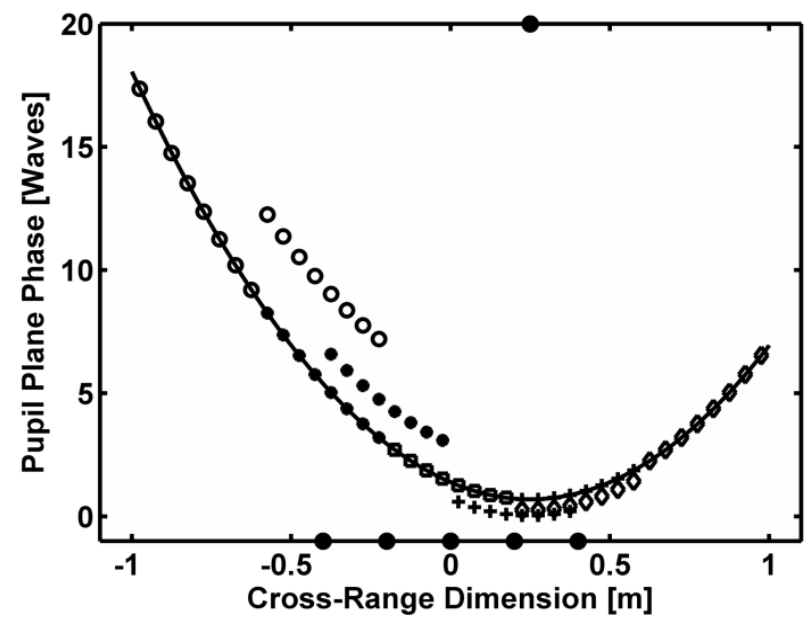

Figure 5: Example of the stripmap HAL transformation applied to five sequentially collected phase only segments resulting from an off-axis point target. In this case the RX aperture effectively overlaps itself by half its diameter during each subsequent TX/RX cycle. Monostatic conditions apply. ${ }^{5}$

\footnotetext{
${ }^{5}$ Reprinted with permission from Applied Optics 48, 1168-1174, (C) Optical Society of America.
} 
By further consideration of the results of Figure 5, in particular the width of the synthetic pupil plane field, we come to the remarkable general conclusion that images created from synthetic pupil plane fields constructed from raw stripmap field segments collected every $D_{a p} / 2$ will have resolutions inversely proportional to an effective aperture diameter $D_{\text {eff-sm }}$ given by

$$
D_{e f f-s m}=2 D_{S A R}+D_{a p} .
$$

In particular, in the virtual focal plane of Figure 1, the image resolution $\triangle C R_{V F P-s m}$ would be, nominally,

$$
\Delta C R_{V F P-s m}=\frac{\lambda f_{V}}{D_{e f f-s m}}=\frac{\lambda f_{V}}{2 D_{S A R}+D_{a p}},
$$

where $f_{V}$ is the focal length assumed for the virtual lens of Figure 1. Of probably greater interest is the longitudinal cross-range resolution $\triangle C R_{s m}$ achievable in the target plane itself. This is found simply by dividing Eq. (13) by the absolute value of the virtual image plane magnification $|M|=f_{V} / R_{0}$ to yield

$$
\Delta C R_{s m}=\frac{\lambda R_{0}}{2 D_{S A R}+D_{a p}} .
$$

Another way of interpreting the results of this section are to define an image sharpening ratio (ISR) that quantifies the image resolution enhancement capabilities of the HAL technique. Simply, the stripmap image sharpening ratio $I S R_{s m}$ is defined as the ratio of the single aperture cross-range resolution to the maximum stripmap HAL crossrange resolution and is given by

$$
I S R_{s m}=\frac{2 D_{S A R}+D_{a p}}{D_{a p}}=\frac{2 D_{S A R}}{D_{a p}}+1 .
$$

In both Eq. (14) and (15) we find that stripmap HAL imaging performance is enhanced by increasing the synthetic aperture diameter $D_{S A R}$. As with traditional stripmap 
SAR and SAL techniques, however, the maximum value of $D_{S A R}$ is limited to the size of the illumination beam footprint in the target plane. This is the case since target plane scatterers at the leading edge of a scene will fully contribute to the HAL data set only if they are illuminated during every shot taken across the entire synthetic aperture [1].

Typically, in the stripmap case the maximum value of $D_{S A R}$ is found by determining the full-width-half-maximum (FWHM) diameter of the illumination beam's intensity profile. As an example, consider the case of a collimated and untruncated Gaussian TX beam at wavelength $\lambda$. Based upon well known Gaussian beam propagation theory, it is quite easy to show that the FWHM (intensity) beam diameter after propagating a distance $R_{o}$ corresponds to a maximum stripmap $D_{S A R}$ of

$$
D_{S A R-\max } \cong 1.2 \omega_{0} \sqrt{1+\left(\frac{R_{0} \lambda}{\pi \omega_{0}^{2}}\right)^{2}},
$$

where $\omega_{o}$ is the TX beam waist and where, as with Eq. (8), far field conditions are not necessarily assumed [19].

Recall that under monostatic ladar conditions the TX beam is nominally untruncated if $\omega_{o}=D_{a p} / 4$. If we then assume realistic values of $D_{a p}=0.1 \mathrm{~m}, \lambda=1.5 \mu \mathrm{m}$ and $R_{o}=30 \mathrm{~km}$, we find that $D_{S A R-\max } \cong 0.7 \mathrm{~m}, \Delta C R_{\text {min-sm }} \cong 3.0 \mathrm{~cm}$ and $I S R_{\text {max }-\mathrm{sm}} \cong 15$. To achieve finer resolution or greater image sharpening a larger synthetic aperture diameter could be achieved by increasing the TX beam divergence. This would, of course, probably come at the expense of having to increase the TX beam power if sub-image signal-to-noise ratios are to be preserved.

Although the results presented above are quite remarkable, we should point out that they are not unexpected. In fact, the maximum achievable stripmap HAL image sharpening ratio, as well as the corresponding minimum sample spacing requirement, are 
essentially the same as for conventional stripmap SAR and SAL sensors where maximum range/cross-range images are desired [1]. 


\section{CHAPTER 3}

\section{PRELIMINARY CONSIDERATIONS}

To demonstrate the HAL processing method's ability to properly phase multiple complex field segments collected across a wide synthetic aperture, careful consideration must be given to the experimental design, including the choice of targets to be examined, as well as the theoretical analyses that will ultimately describe the behavior of our experimental HAL system. These topics will, in part, be explored in this section.

\subsection{Experimental Design}

The targets chosen for our experiment were a set of single frequency, diffusely reflecting Ronchi rulings at discrete spatial frequencies chosen to allow sufficient sampling across the spatial frequency spectra of both a diffraction limited single sub-aperture, as well as a fully HAL processed effective aperture. As we will demonstrate, this choice of target set conveniently allowed us to map out the MTF for both a single sub-aperture, as well the fully HAL processed effective aperture.

Our 50\% duty cycle Ronchi ruling targets were composed of parallel chrome bars deposited edge-to-edge on clear two inch square glass plates. During data collection these were backed by a high reflectivity anodized aluminum block whose texture was found to be highly diffuse at our source wavelength. In our experimental work the slides were 
illuminated in such a way as to ensure that specular reflections from the chrome bars fell outside the field of view of our receiver aperture. To a distant observer, then, the chrome bars yielded the opaque bands of a typical Ronchi ruling, with bright diffuse bands resulting from the anodized aluminum block lying in between. A top-down view of our general experimental setup is shown in Figure 6.

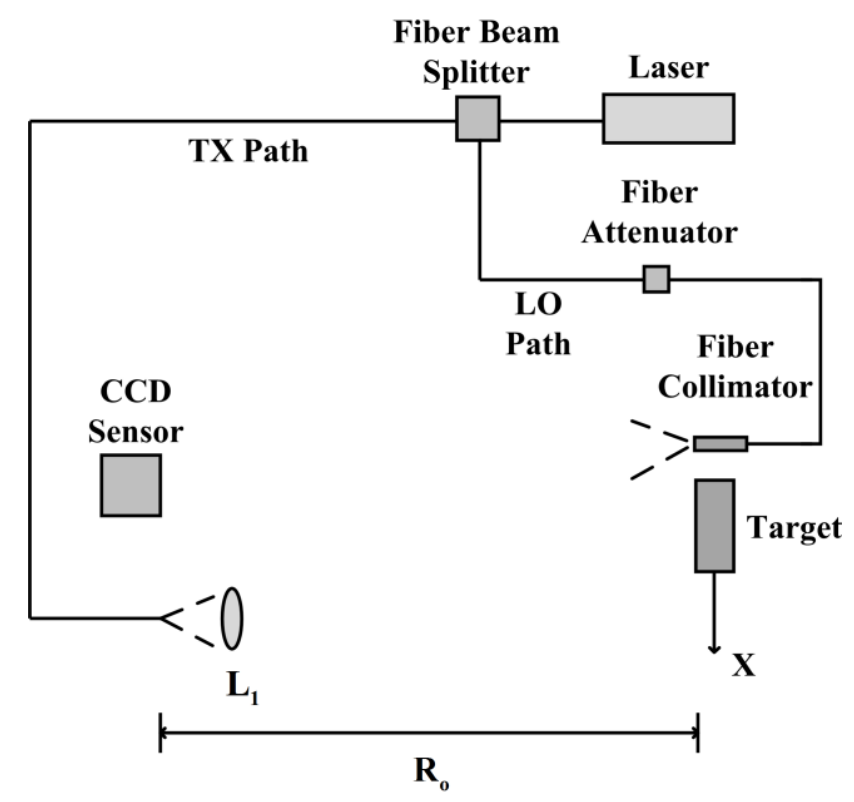

Figure 6: Diagram of our experimental setup.

An $80 \mathrm{~mW}$ fiber coupled IR laser, with wavelength $\lambda=1.545 \mu \mathrm{m}$, was sent through a fiber beam splitter to form both the transmitter (TX) and local oscillator (LO) beams. The TX path consisted of a bare, cleaved, single mode fiber with a core diameter of $8 \mu \mathrm{m}$, while the LO path, also single mode, included an in-line fiber collimator (to reduce $\mathrm{LO}$ beam divergence) placed below the target at a location that would not interfere with later target translation. Tip/tilt adjustments were manually applied to the LO in order to uniformly illuminate the $\mathrm{CCD}$, and an in-line fiber attenuator was placed in the $\mathrm{LO}$ beam path in order to precisely control the intensity of the beam and prevent it from 
causing detector saturation. In addition, the LO beam exit aperture was fixed in the plane of the target while the TX exit aperture, in accordance with one of the fundamental assumptions of HAL processing methods, was fixed in the plane of the bare CCD sensor (i.e., the receiver (RX) aperture pupil of our system) [2]. The LO beam location was chosen to simplify both the laboratory setup and data processing steps, as well as to eliminate aberrations that might be introduced if a beam splitter mixing configuration was instead employed close to the detector. For example, at our source wavelength the beam waist diameter at the collimator output was approximately $0.44 \mathrm{~mm}$, yielding a Rayleigh range of just under $10 \mathrm{~cm}$. As this was much smaller than our nominal range to target, the range phase curvature of both the LO beam and the back scattered signal beams were matched, thereby eliminating the need to account for range phase mismatches in post processing.

In general it is the HAL sensor and the TX which are in motion as the TX beam sweeps across the distant target [2]-[3]. For our laboratory work, however, we found it convenient to employ the principle of relative motion and instead translate our targets. That is, the CCD and the TX and LO beam positions remained fixed, while the target, attached to translation stage with micrometer screw adjustment capability, was moved to different locations across the synthetic aperture as sequential frames of scattered field data were collected in the plane of the CCD.

The TX beam height above our optical table was made somewhat smaller than the height of the target in order to deflect away specular reflection components arising from the chrome bars of the Ronchi ruling targets, as well as the underlying glass substrate. In addition, a $10 \mathrm{~mm}$ diameter cylindrical lens $L_{1}$ with a focal length of $f_{1}=20 \mathrm{~mm}$ was 
placed in front of the TX beam aperture and slightly defocused in order to constrain the expanding beam in the vertical direction while also allowing it to freely diffract in the horizontal direction (i.e., in the plane of the table). This was done in order to maximize signal return from the targets, while also providing a wide and more or less uniform flood illumination beam footprint in the plane of the target, at least across the target's full range of motion in the cross-range dimension. In the plane of the target, our illumination beam $\left(1 / \mathrm{e}^{2}\right)$ measured approximately $46 \mathrm{~cm}$ wide by $8 \mathrm{~cm}$ high.

Our CCD was the bare sensor from a FLIR, Inc., SC2500 series camera. The sensor included an array of 320 x 256 pixels (later digitally cropped to 256 x 256) with a pixel pitch $p_{x}$ of $30 \mu m$. The Ronchi ruling targets were (sequentially) placed across our optical table at a distance $R_{o}$ from the CCD, which we carefully measured to be $3.59 \mathrm{~m}$. Finally, the centers of the targets and the CCD were positioned at identical heights above our optical table and the faces of the targets and the CCD were carefully adjusted to be parallel.

A picture of the portion of the setup containing the target can be seen in Figure 7. This figure shows the vertical chrome bars of the $0.25 \mathrm{cyc} / \mathrm{mm}$ target that is mounted to the diffuse anodized aluminum block with the use of two fastening clamps. The target and holding apparatus were then mounted to a linear micrometer positioning stage that allowed for precise movements of the target. The apparatus to the left of the target contained the local oscillator output. The LO fiber output was attached to post that allowed adjustments in the range dimension in efforts to place the output in the plane of the target. It can also be seen that the LO unit was attached to the optical table and did not translate with the target object. 


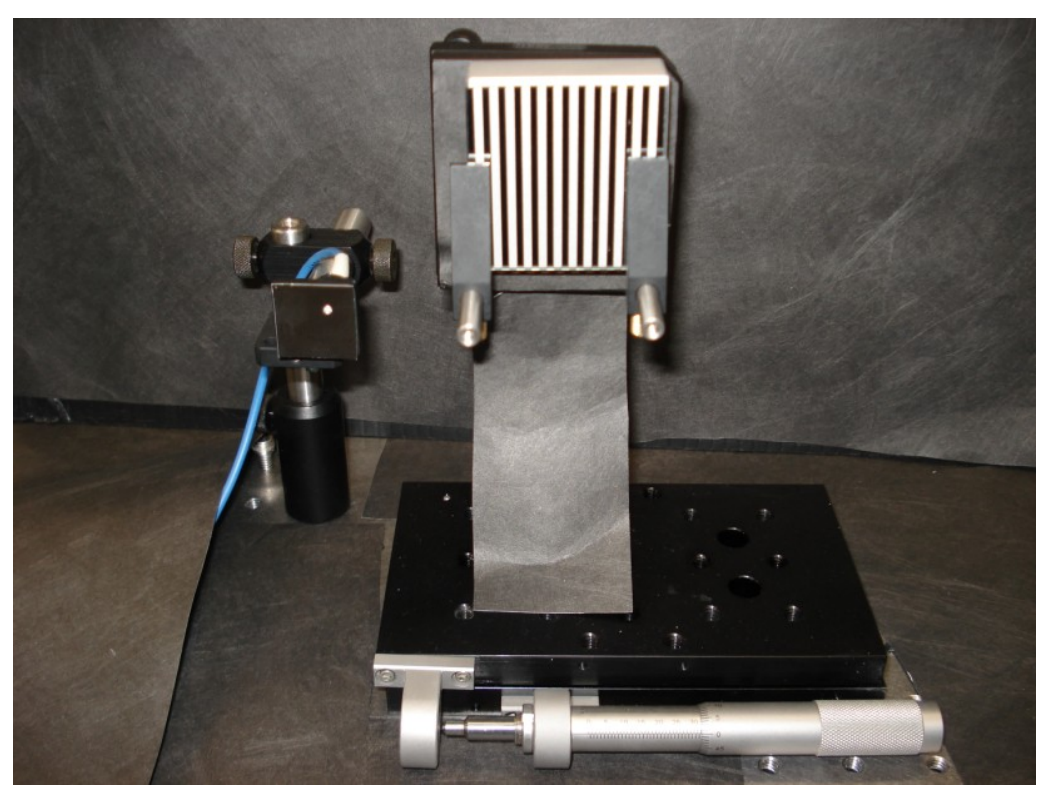

Figure 7: Lab setup containing the target object, $(0.25 \mathrm{cyc} / \mathrm{mm}$ as shown) translation stage and local oscillator unit.

\subsection{The Image Sharpening Ratio and Spatial Bandwidth}

The incoherent, diffraction limited spatial frequency bandwidth $f_{o}$ of an image formed from a single sub-aperture frame of data can be found from the following equation

$$
f_{0}=D_{a p} / \lambda R_{0},
$$

where $D_{a p}$ is the width of the cropped CCD RX array [19]. With digital cropping to 256x256 pixels, our pixel pitch yields a $D_{a p}$ of $7.68 \mathrm{~mm}$. Based upon our target range and wavelength, equation (17) then yields a single sub-aperture bandwidth of $1.38 \mathrm{cyc} / \mathrm{mm}$.

The effective aperture width, after full HAL processing, is a direct function of how many sub-aperture frames of data are used, as well as the interframe overlap between neighboring frames. We have shown previously that neighboring frames of data, after Stripmap HAL processing, will be just contiguous if they were collected with relative interframe motion $\Delta_{x}$ of exactly Dap/2 [2]. In practice, though, when examining 
diffuse targets we have found it helpful to use an interframe relative motion of something less than Dap/2. While oversampling does not directly increase the resolution of fully HAL processed imaged, it does allow commonly known speckle field correlation techniques (to be described in detail later) to be employed to precisely align the overlapping regions of neighboring frames of HAL processed data, thereby preserving the potential to increase image resolution.

For our work we chose to have an interframe relative motion of $\Delta_{x}=2 \mathrm{~mm}$, yielding neighboring frames of data that overlapped by a bit less than half. The effective aperture width $D_{E F F}$ is then found according to

$$
D_{E F F}=\left[2 \frac{\Delta_{x}}{D_{a p}}(N-1)+1\right] D_{a p},
$$

where $N$ represents the number of sub-aperture frames of data collected. Moreover, from equation (18) the potential HAL processed image sharpening ratio (ISR) can be found according to [2]

$$
I S R=\frac{D_{E F F}}{D_{a p}}=\left[2 \frac{\Delta_{x}}{D_{a p}}(N-1)+1\right]
$$

For our work we collected 12 frames of data for each Ronchi ruling target, taking great care to translate our target exactly $2 \mathrm{~mm}$ between neighboring frames. This resulted in a synthetic aperture of $D_{E F F}=51.68 \mathrm{~mm}$ and a potential maximum image sharpening ratio of $I S R=6.73$. This corresponds to a potential spatial bandwidth of $9.29 \mathrm{cyc} / \mathrm{mm}$ after full HAL processing. 


\subsection{MTF Measurement}

The modulation transfer function (MTF) is a quantitative measure of object-to-image contrast transfer as a function of spatial frequency [19]. To examine the image sharpening capabilities of the stripmap HAL process, one of our central goals was to produce MTF curves demonstrating the bandwidth and image contrast decrease as a function of spatial frequency for images formed from both single and HAL processed multiple frames of data. In all cases, experimental MTF curves were constructed by taking the two dimensional Fourier transform of single or multiple frame final images of each Ronchi ruling target, and then plotting normalized values of the fundamental spatial frequency peaks. In order to determine the appropriate normalization constant, though, we must first consider the Ronchi ruling targets themselves a bit more carefully.

The Ronchi ruling grating target is composed of a high edge definition, alternating pattern of chrome and glass bars. An idealized cross section of the intensity $I(x)$ of our $50 \%$ duty cycle Ronchi ruling target images is shown in Figure 8, where $A$ is the mean image intensity, the peak-to-peak intensity variation is $2 \mathrm{~B}$ and where the spatial period is $W_{R}$.

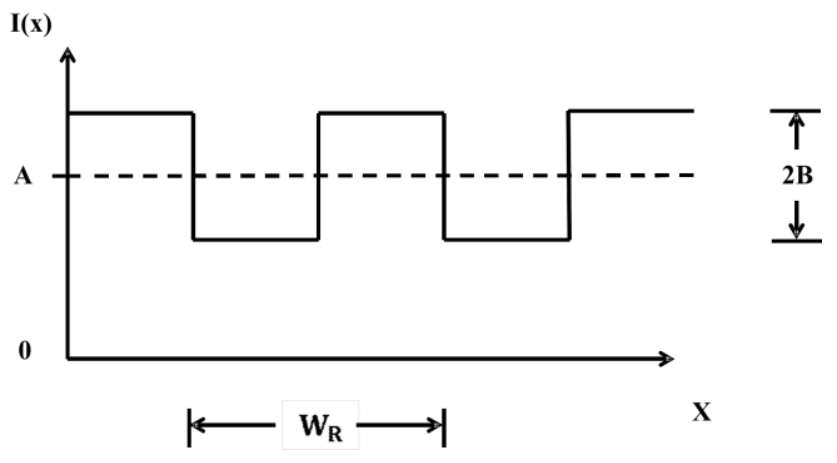

Figure 8: An idealized image intensity cross section for a $50 \%$ duty cycle Ronchi ruling. The mean intensity is $A$, the peak-to-peak variation is $2 B$ and the spatial period is WR. 
The contrast, or visibility, $V$ of the target is then found to be

$$
V=\frac{I_{\max }-I_{\min }}{I_{\max }+I_{\min }}=\frac{(A+B)-(A-B)}{(A+B)+(A-B)}=\frac{B}{A} .
$$

In addition, the Ronchi ruling image cross section can be modeled through a Fourier series representation of $I(x)$ according to the relationship

$$
I(x)=A+B \sum_{n=-\infty}^{\infty} F_{n} \exp \left(j n p_{0} x\right)
$$

where the Fourier series coefficients $F_{n}$ and fundamental frequency $p_{o}$ are given by

$$
F_{n}=S a\left(n \frac{\pi}{2}\right)
$$

and

$$
p_{o}=\frac{2 \pi}{W_{R}}
$$

respectively, and where the sine-over-argument $S a$ function in equation (22) is defined as $\operatorname{Sa}(\mathrm{x})=\sin (\mathrm{x}) / \mathrm{x}[21]$. The Fourier transform of $I(x)$, in one dimension, can then be shown to be,

$$
I(p)=2 \pi A \delta(p)+2 \pi B \sum_{n=-\infty}^{\infty} F_{n} \delta\left(p-n \omega_{0}\right)
$$

where $\delta$ represents the Dirac delta function, and where $p$ is the general radian spatial frequency variable.

Now, the amplitude of the fundamental spatial frequency term (i.e., $n=1$ ) of equation (24) is readily found to be $\mathrm{c}_{l}=(2 \pi B) 2 / \pi=4 \mathrm{~B}$, while the zero spatial frequency 
term's amplitude is simply $c_{o}=2 \pi A$. The fundamental and zero frequency amplitudes can then be used to determine the image visibility according to

$$
V=\frac{\pi}{2} \frac{c_{1}}{c_{0}}
$$

Dividing the image visibility by the original target visibility $V_{o}$, the MTF value at spatial frequency $f_{x}=1 / W_{R}$ can then be expressed as,

$$
\operatorname{MTF}\left(f_{x}\right)=\frac{V}{V_{0}}=\frac{1}{V_{0}} \frac{\pi}{2} \frac{c_{1}}{c_{0}} .
$$

Since our Ronchi ruling targets were of such high contrast, in all work which follows we simply set $V_{o}$ to unity. We thus see that by determining the zero and fundamental frequency peaks for a collection of Ronchi ruling images, the MTF of our system can be readily determined. 


\section{CHAPTER 4}

\section{TARGET SIMULATIONS}

In efforts to produce results that were obtained in a laboratory environment, it would first be beneficial to perform simulations to determine what could be expected. Due to the simplicity of a single frequency Ronchi ruling target; multiple targets could be readily simulated using a simple processing algorithm. In this section, processing steps will be thoroughly explained that begin with the digital creation of the targets to the final step of plotting an MTF line. Experimental laboratory values were used in the formation of the MTF plots. A visual analysis of the effects of speckle degradation on image quality is provided depicted by the multiple targets used for the creation of both the sub-aperture and synthetic images are provided. Finally, the simulated sub-aperture and synthetic MTF will be created and explored.

\subsection{Simulation Steps}

The goal of this section is to provide an algorithm that will successfully produce simulation results that accurately model the laboratory results that were later obtained. Figure 9 details the general processing steps that were taken to produce the MTF plots. In this figure, the processing steps are clearly indicated while in some boxes, the upper left 
corner will display a $V T, P$ or $I$ representing the virtual target, pupil or image plane respectively.

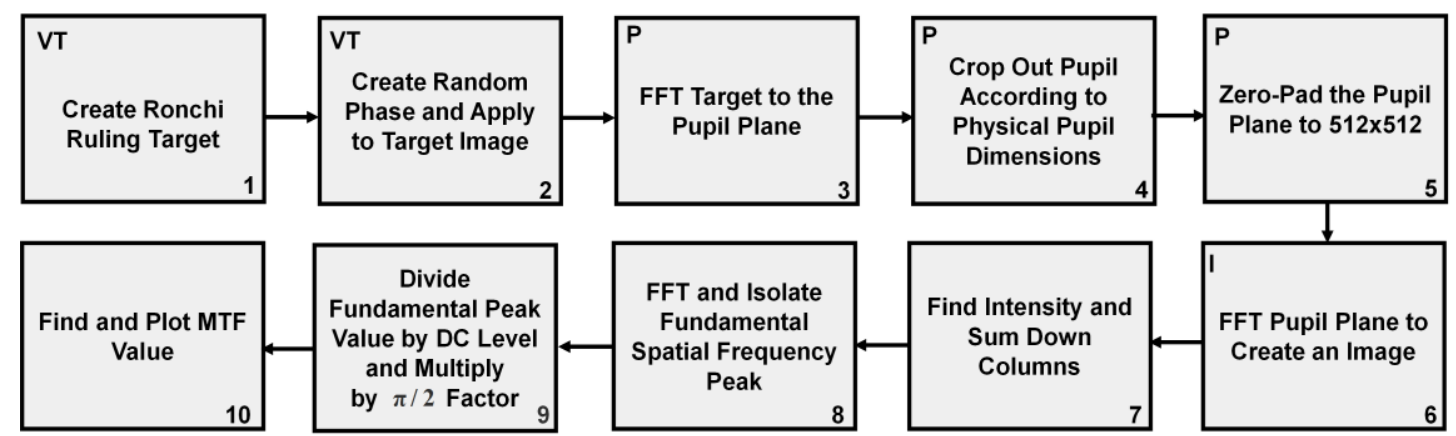

Figure 9: Simulation processing steps.

The first step in the process was to digitally create the specific Ronchi target that was to be used in the simulation and due to the simplicity of this specific target, can be readily created. An example of a $0.25 \mathrm{cyc} / \mathrm{mm}$ target can be seen in Figure 10 .

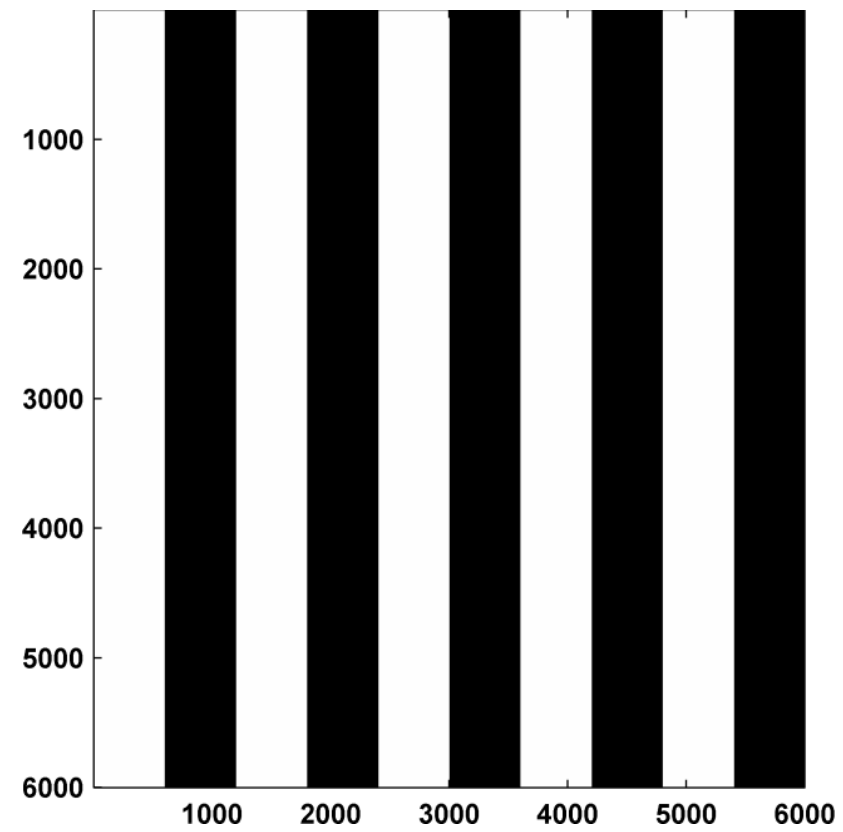

Figure 10: Example of a $0.25 \mathrm{cyc} / \mathrm{mm}$ target object that was created.

The initial array created was a 6000x6000 matrix which was the largest size array that we were able to process. This was performed to give each bar enough points in 
efforts to avoid the final image looking under-sampled. By initially allocating a set array size, the spacing between points will be the same and the number of points per bar for each specific target will vary.

The next step was to apply a random phase to the entire target array. This was performed using a uniformly distributed random number generator and multiplying the target object array in the form of equation (27) where $O^{\prime}\left(x_{o}, y_{o}\right)$ is the target object field after the random phase is applied

$$
O^{\prime}\left(x_{o}, y_{o}\right)=O\left(x_{o}, y_{o}\right) \exp \left(j 2 \pi \varphi_{\text {Rand }}\right) .
$$

The random phase that was applied will help to simulate the rough surface of the target (i.e. with respect to wavelength) and will manifest itself as speckle in the final image.

The next step in the process was to perform a Fourier transform on the target object image and propagate it to the pupil plane. At this point, the very large array is then cropped to simulate the real pupil aperture of the experimental setup. This was accomplished by dividing the real aperture width by the pupil plane pixel size as dictated by experimental design parameters. For example, for the experimental sub-aperture with a pixel pitch of $30 \mu \mathrm{m}$ and $\mathrm{CCD}$ array size of 256 pixels, the aperture width was found to be $D_{a p}=7.68 \mathrm{~mm}$. The propagation induced pupil plane pixel size was then found to be $\frac{\lambda z}{D_{T}}=277.3 \mu \mathrm{m}$ where the wavelength used was $\lambda=1.545 \mu \mathrm{m}$, the range $z=3.59 \mathrm{~m}$ and the physical target diameter was $D_{T}=20 \mathrm{~mm}$. It is clearly seen that the ratio of the real aperture diameter over the propagation induced pixel size will produce units of pixels only. This pixel value dictated by the experimental design parameters will be the size of the array that will be cropped out of the initial large pupil array. For the design 
parameters listed above, the aperture size cropped out initial pupil array was $28 \times 28$ pixel array.

The next step in the simulation was to zero-pad the cropped, pupil array to $512 \times 512$ pixels. A Fourier transform of the zero-padded array will then form an upsampled final image. This process will produce an image with the effects of speckle degradation on the final image. An example of a final image can be seen in Figure 11. This figure is that of a $0.25 \mathrm{cyc} / \mathrm{mm}$ target that was used in the formation of the simulated sub-aperture MTF meaning that the simulated pupil aperture was used was the same $28 \times 28$ pixel array described above.

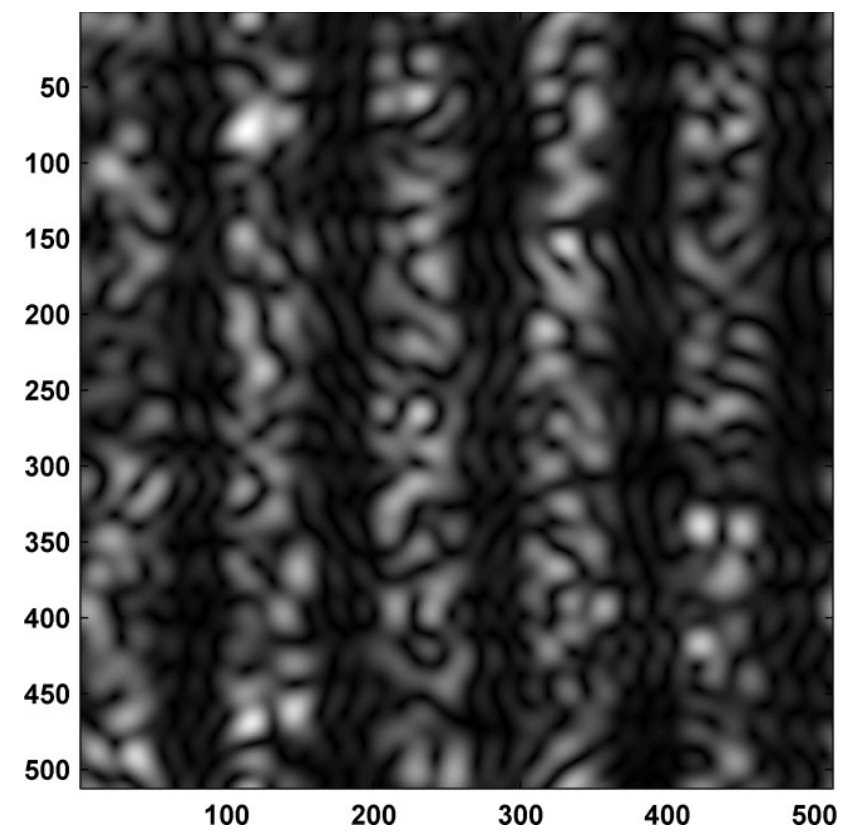

Figure 11: Final image of a $0.25 \mathrm{cyc} / \mathrm{mm}$ target used in the construction of the sub-aperture MTF.

The final simulated image was next converted to intensity via modulus squared and the columns of the image were summed to effectively perform a speckle averaging of the final image. This step of speckle averaging the intensity image will again be explored in the processing sections of Chapter 5. The next step is to perform a Fourier transform of 
the speckle averaged, intensity image. This will produce the spatial frequency harmonics along with a zero frequency value that are required for the MTF plot. The array is then normalized by the DC level value. An example of the spatial frequency cross section plot for a $0.25 \mathrm{cyc} / \mathrm{mm}$ target image is shown in Figure 12 .

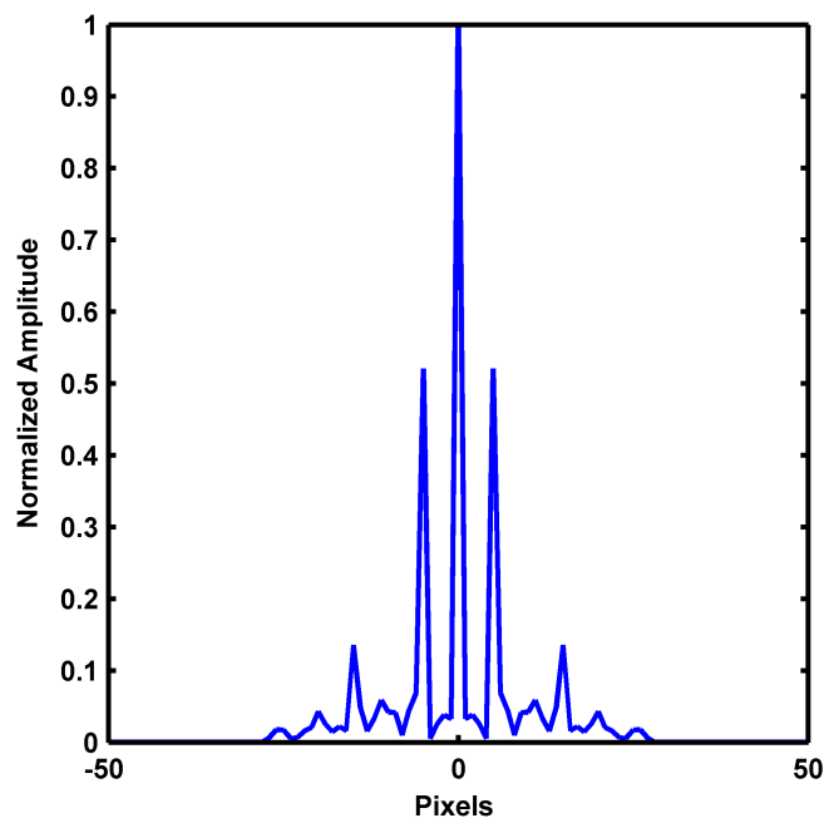

Figure 12: Example of a cross section plot for $0.25 \mathrm{cyc} / \mathrm{mm}$ target.

The first harmonic value can then be obtained and multiplied by the $\pi / 2$ factor that was discussed in Chapter 3. This value can then be plotted on the simulated MTF line and the entire process repeated form multiple targets that were used.

\subsection{Sub-Aperture Simulations}

In efforts to produce the simulated sub-aperture MTF, multiple Ronchi targets were used that were below the diffraction limited spatial frequency cut-off of $1.38 \mathrm{cyc} / \mathrm{mm}$. In all, five targets were created and processed. The visual results for all targets are shown below. It should be noted that the $0.25 \mathrm{cyc} / \mathrm{mm}$ final simulated image was previously shown to demonstrate the processing flow chart, and can be seen in Figure 11. The four 
other final simulated images correspond to $0.50,0.75,1.00$ and $1.25 \mathrm{cyc} / \mathrm{mm}$, respectively, and where speckle degradation of the image is highly apparent.

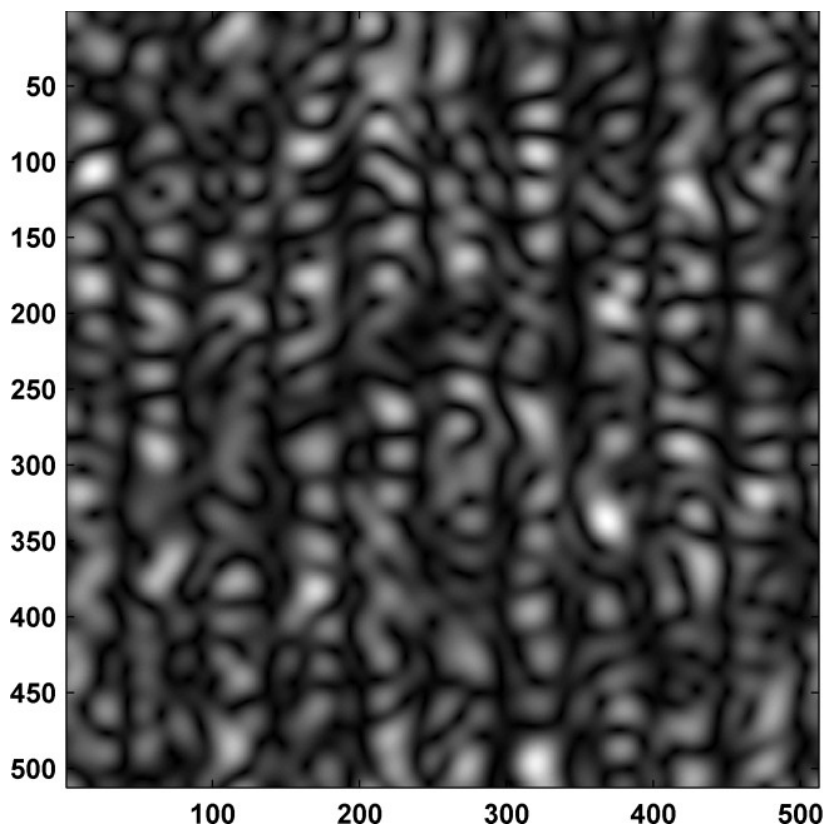

Figure 13: Final image of a $0.50 \mathrm{cyc} / \mathrm{mm}$ target used in the construction of the sub-aperture MTF.

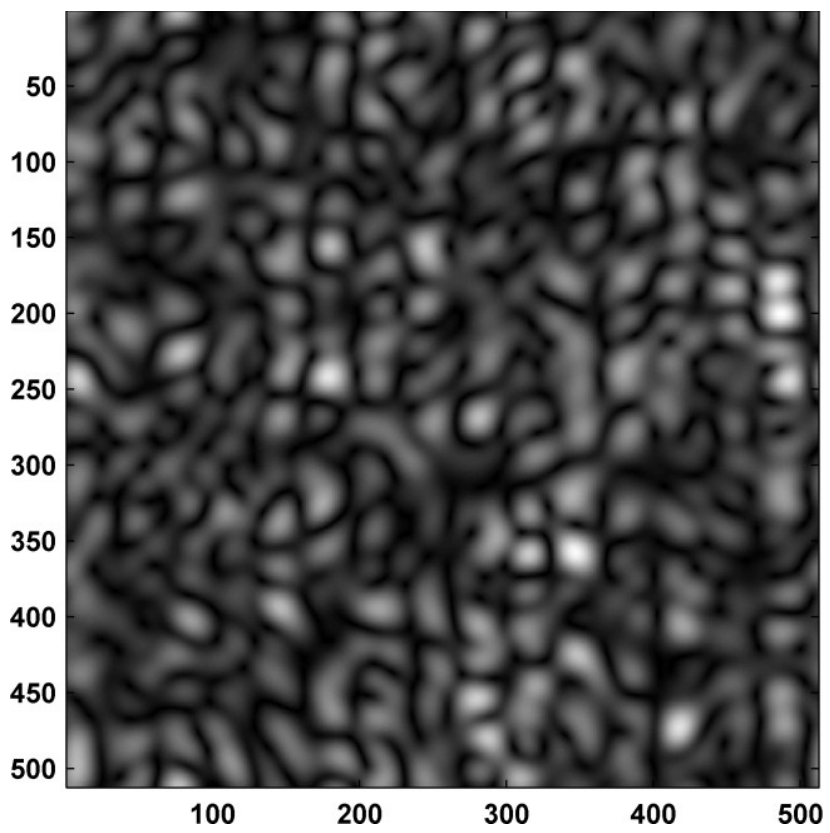

Figure 14: Final image of a $0.75 \mathrm{cyc} / \mathrm{mm}$ target used in the construction of the sub-aperture MTF. 


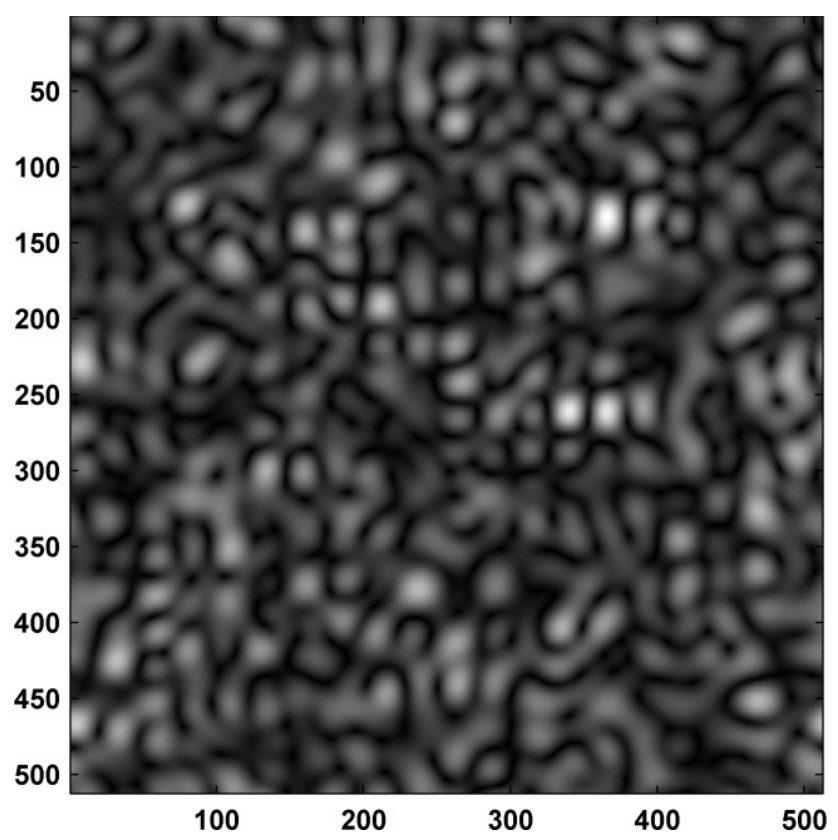

Figure 15: Final image of a $1.00 \mathrm{cyc} / \mathrm{mm}$ target used in the construction of the sub-aperture MTF.

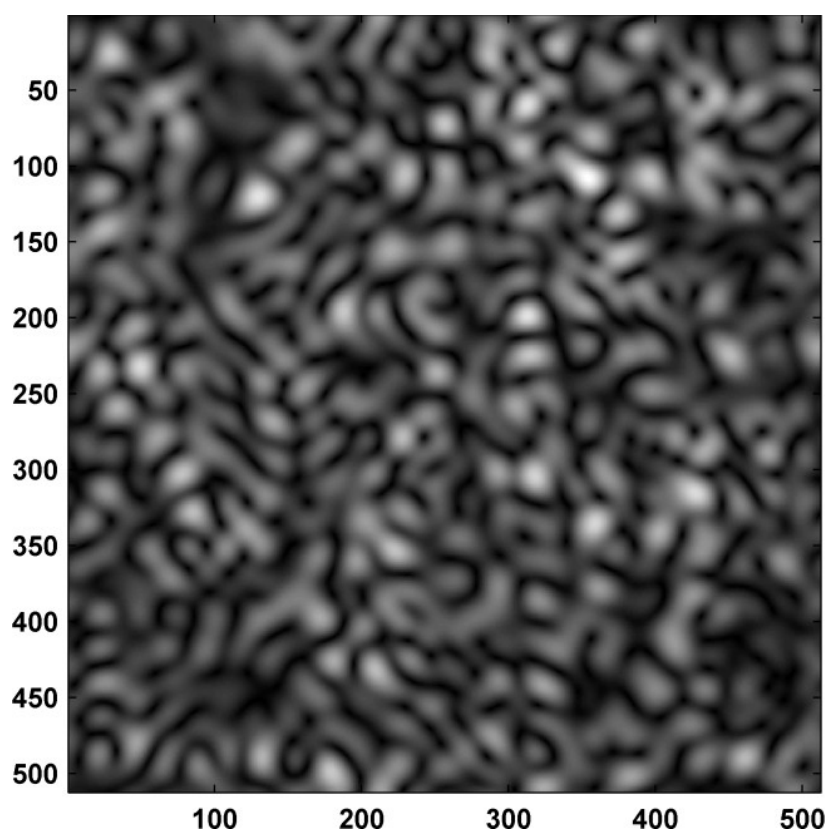

Figure 16: Final image of a $1.25 \mathrm{cyc} / \mathrm{mm}$ target used in the construction of the sub-aperture MTF

The results in Figure 17 display the simulated sub-aperture MTF where the data points for each specific target are shown and best fit line was plotted through the data 
points. The cut-off value was found to be $1.375 \mathrm{cyc} / \mathrm{mm}$ which closely matches the theory.

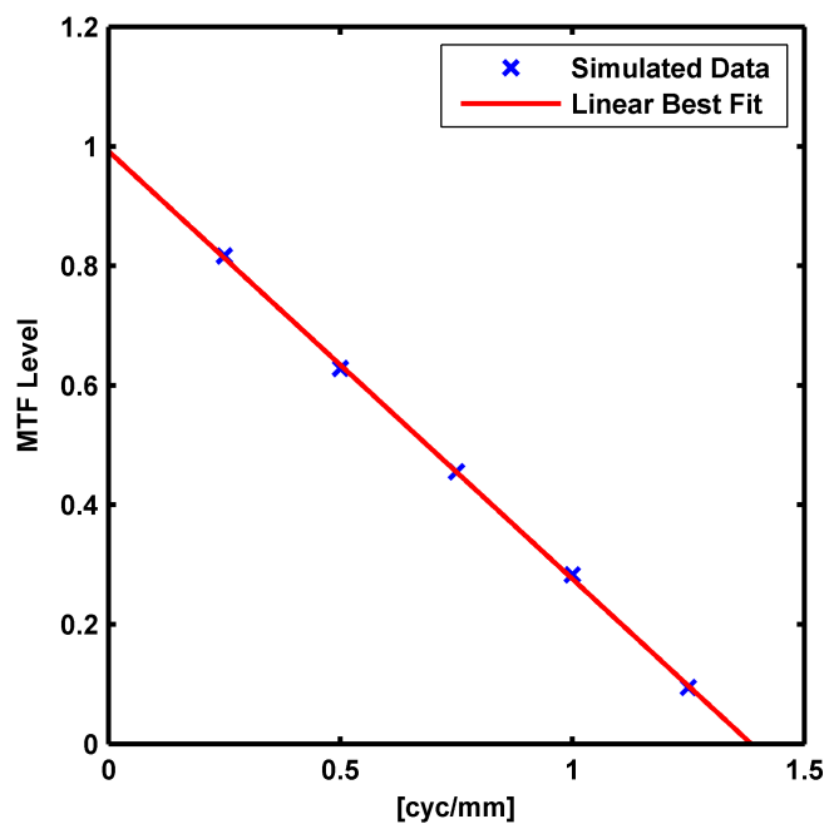

Figure 17: Simulated sub-aperture image MTF.

\subsection{High Resolution Synthetic Image Simulations}

The simulation processing steps used to create the simulated sub-aperture MTF were also used to create the simulated synthetic MTF. The same experimental design parameters were used while the effective aperture diameter was replaced for the sub-aperture diameter when calculating the initial pupil crop of step 4 in Figure 9. The effective aperture diameter of $D_{\text {eff }}=51.6 \mathrm{~mm}$, explained in section 3.2, corresponded to a crop of $28 \times 186$ pixels. It should be noted that simulating the effective aperture increases the horizontal direction only. This simulation, of course, is analogous to the effective aperture increasing in one dimension due to multiple sub-apertures that are overlapped. Steps 5-10 in Figure 9 are then followed to produce a data point. Five targets were 
created to produce the MTF plot and the final simulated images are shown below and correspond to $1,2,4,6,8 \mathrm{cyc} / \mathrm{mm}$.

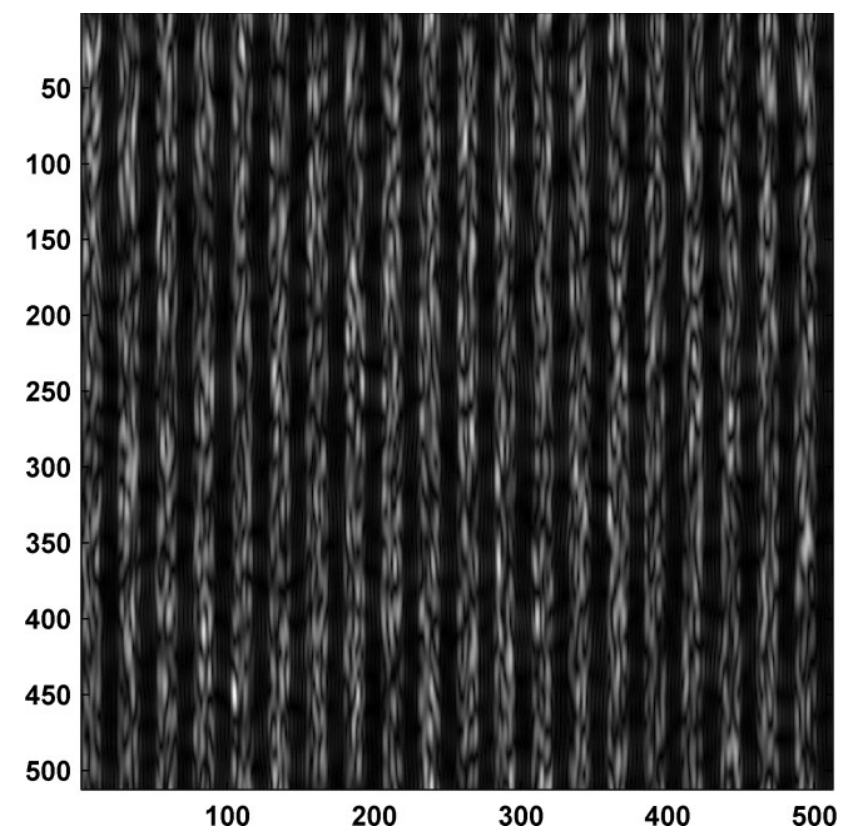

Figure 18: Final image of a $1.00 \mathrm{cyc} / \mathrm{mm}$ target used in the construction of the synthetic image MTF.

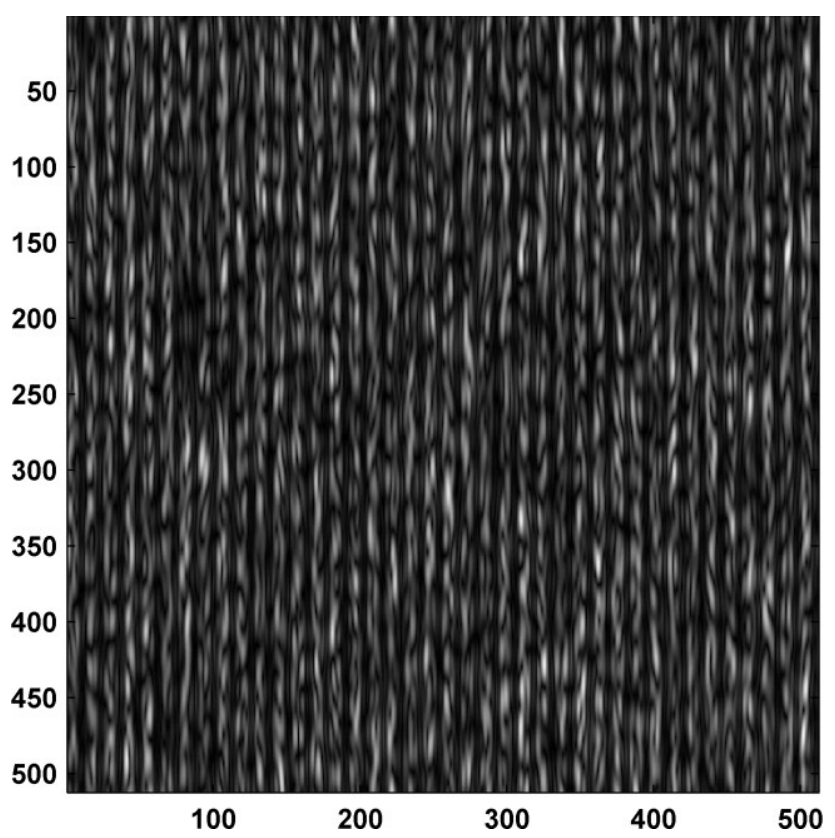

Figure 19: Final image of a $2.00 \mathrm{cyc} / \mathrm{mm}$ target used in the construction of the synthetic image MTF. 


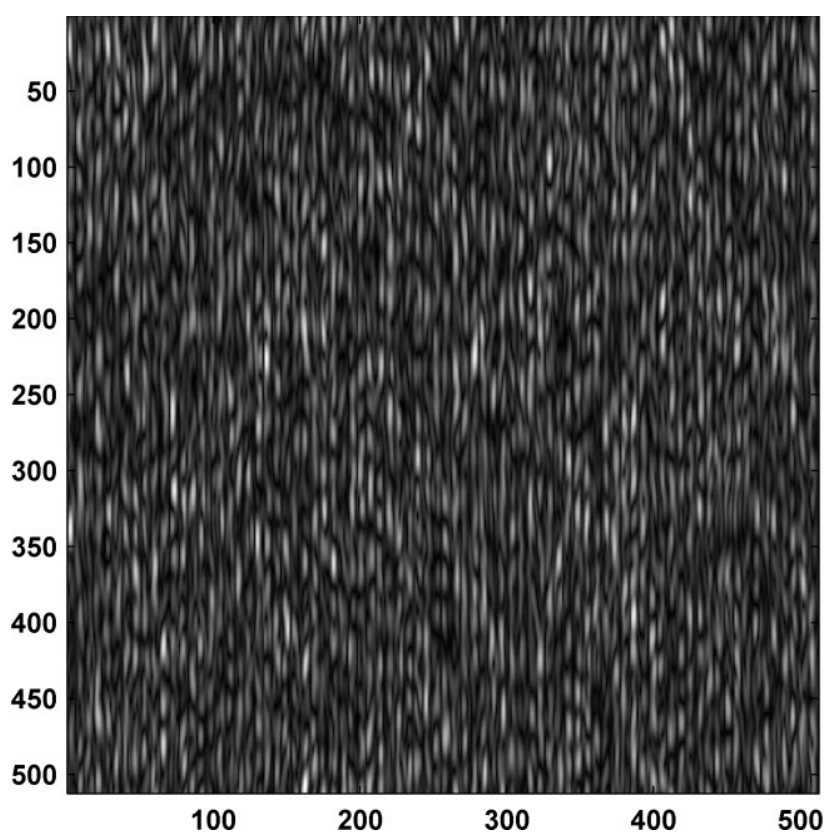

Figure 20: Final image of a $4.00 \mathrm{cyc} / \mathrm{mm}$ target used in the construction of the synthetic image MTF.

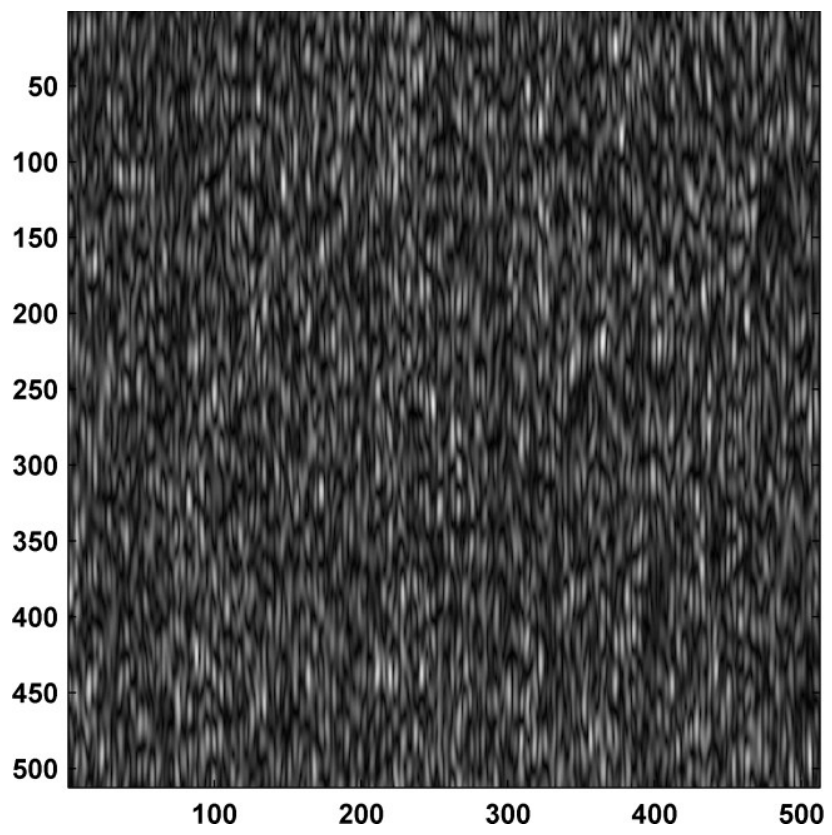

Figure 21: Final image of a $6.00 \mathrm{cyc} / \mathrm{mm}$ target used in the construction of the synthetic image MTF. 


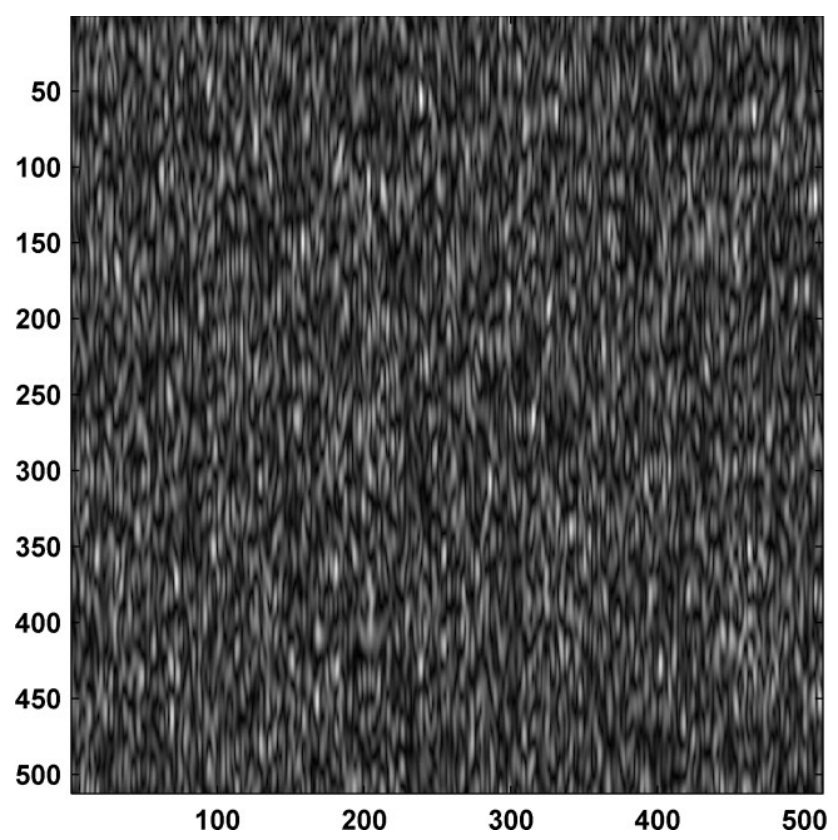

Figure 22: Final image of an $8.00 \mathrm{cyc} / \mathrm{mm}$ target used in the construction of the synthetic image MTF.

For the plot in Figure 23, the simulated synthetic MTF was formed. The theoretical cut-off for the HAL processed MTF was found to be $9.29 \mathrm{cyc} / \mathrm{mm}$ and the simulated plot shown below closely matches this value with a cut-off of $9.24 \mathrm{cyc} / \mathrm{mm}$.

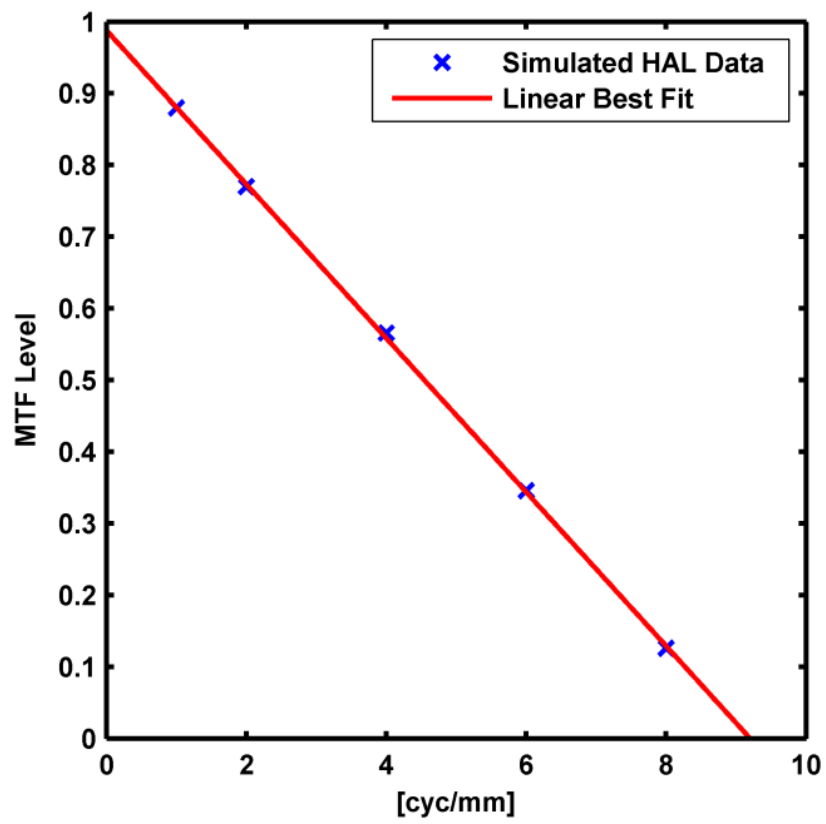

Figure 23: Simulated synthetic image MTF. 


\section{CHAPTER 5}

\section{DATA COLLECTION AND PROCESSING}

For the work detailed in this chapter, sub-aperture frames were collected in the lab via off-axis digital holograms at multiple target locations. Stripmap HAL theory dictates that the transmitter and receiver move in synchronous, constant motion. This is achieved by moving the target object while fixing the TX/RX. The target object was chosen to be a Ronchi type grating of single frequency, therefore multiple Ronchi ruling targets would have to be used to construct an MTF plot. Theoretical calculations would define cut-off values for both sub-aperture and HAL processed limits. Prior work performed in this area proved the Stripmap HAL transform's ability to account for phase errors collected across a effective aperture for a simple point target. However, the next step was to show that the Stripmap HAL transform could account for phase errors of an extended, complex target. This led to the formation of the HAL processing method which details all the steps taken to produce the HAL processed MTF. This plot would then quantitatively show the resolution gains that were achieved past the single, sub-aperture diffraction limit. The sub-aperture processing steps are also included which detail the steps taken to produce the sub-aperture MTF. 


\subsection{Sub-Aperture Processing Steps}

In efforts to show the resolution gains resulting from the HAL processed MTF, a baseline single sub-aperture MTF plot would be useful to visually show the improvements that were made by successfully stitching together a pupil of larger extent. For the work involved in this section, sub-aperture frames were collected at multiple spatial frequencies below the cut-off frequency in efforts to produce an MTF plot. Multiple realizations at each target frequency would allow an error bar plot to be produced to ensure the repeatability of the process. Table 4 , located at the end of section 5.2, contains the sub-aperture data that was processed. Also, a sub-aperture processing flow chart was created to detail the steps that were taken to produce the MTF plot. This process contains a subset of steps that correspond with the HAL processing flow chart. The sub-aperture flow chart can be found below in Figure 24. Some of the steps in the process will have a $P$ or $I$ in the top left corner corresponding to pupil and Image and indicate in which plane the action is taking place. A detailed description of the sharpening algorithm, which was used in the sub-aperture processing steps, will be discussed in section 5.2.

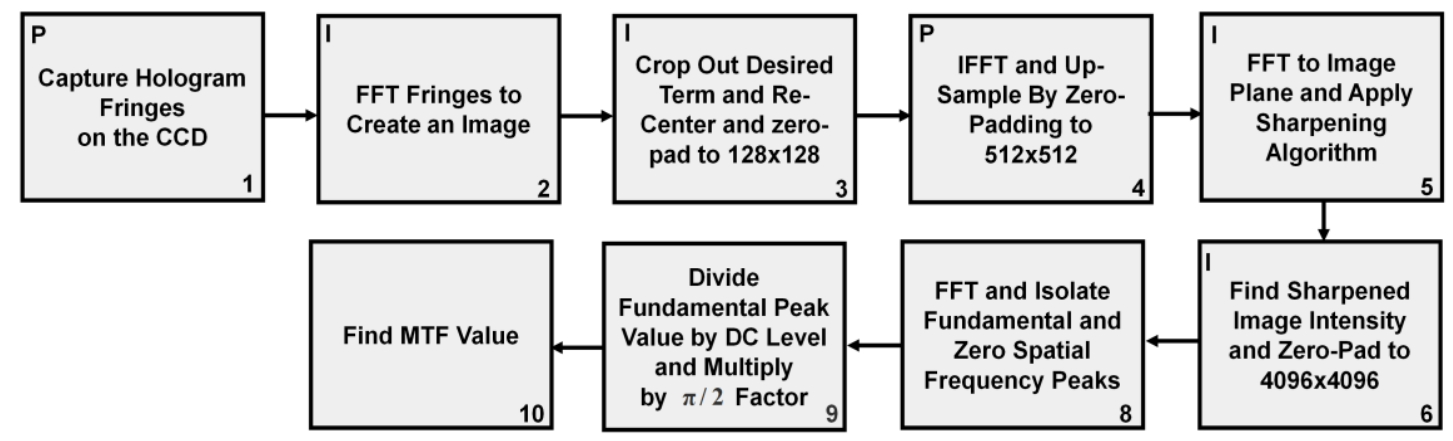

Figure 24: Sub-aperture processing steps.

The first step is to capture fringes on the CCD where $g_{i}^{\prime}(x, y)$ is the target field information and $f_{L O}(x, y)$ is the field of the local oscillator of the form, 


$$
\left|g_{i}^{\prime}(x, y)+f_{L O}(x, y)\right|^{2}=\left|g_{i}^{\prime}\right|^{2}+\left|f_{L O}\right|^{2}+g_{i}^{\prime} f_{L O}^{*}+g_{i}^{*} f_{L O}
$$

The two fields mix on the CCD where they are converted to intensity and yield the first two autocorrelation terms. The third term contains the desired target information while the last term is the complex conjugate of the target information.

The next step is to Fourier transform the intensity fringes to create an image. Due to the fact that the LO was placed in plane of the target, the Fourier transform of the pupil plane data will produce a target image and conjugate image that are in focus. Figure 25 depicts the focused target image, which was taken to be the first quadrant and a crop of the target information. This was performed due to the fact that target holding apparatus extended out of the plane of the target. Therefore, the central portion of the Ronchi grating was cropped in the image plane to eliminate the unwanted characteristics as well as the overall noise present in the frame. Also note the noise surrounding the targets due to the flood illumination of the TX beam.
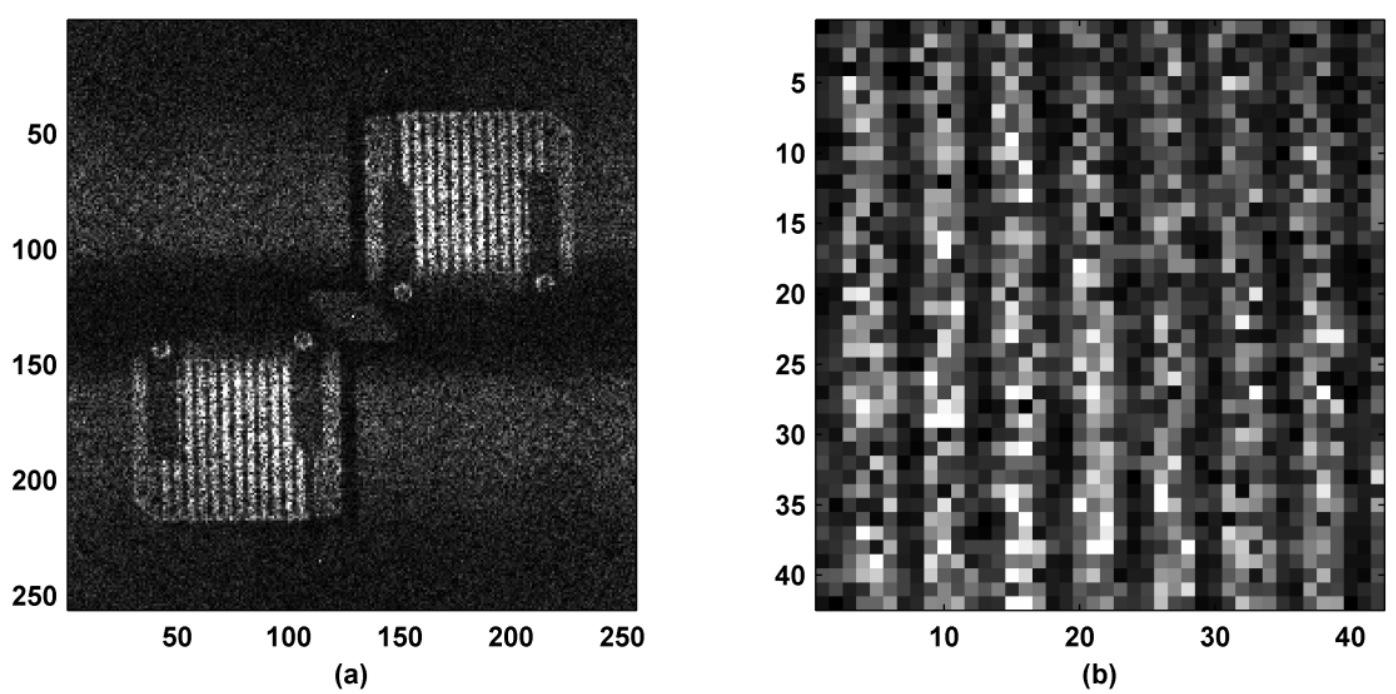

Figure 25: Image plane target information (a) and a crop of the target information. 
The size of the target crop was taken to be $46 \times 46$ pixels where the vertical bars of the $0.25 \mathrm{cyc} / \mathrm{mm}$ Ronchi ruling are clearly visible.

The next step is to zero-pad the cropped image segment to $128 \times 128$ pixels, which was half the size of the CCD sensor array. Next, an inverse Fourier transform propagated the array to the pupil plane and the segment was zero-padded to $512 \times 512$ pixels. A Fourier transform of the zero-padded pupil segment will produce an up-sampled image. This was performed to preserve the resolution of the target image and does not increase resolution. An example of the up-sampled image can be seen in Figure 26.

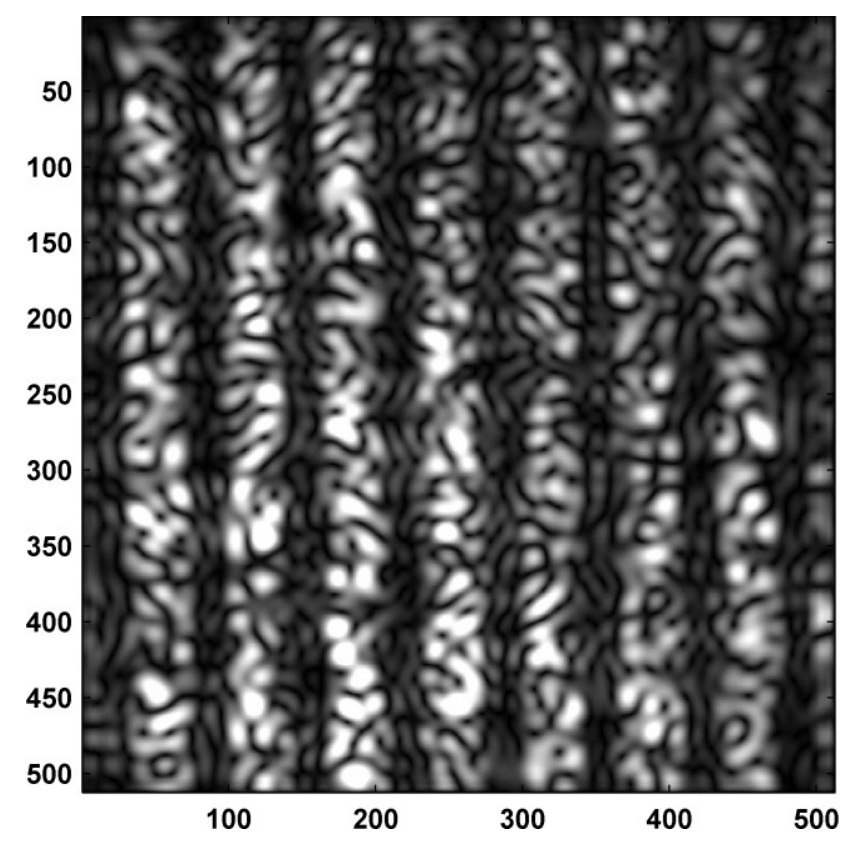

Figure 26: Up-Sampled 512x512 image.

The speckle on the up-sampled image is highly evident in Figure 26 and results from the rough surface of the target. The complex, up-sampled image will next be converted to an intensity image and will then be zero-padded to 4096x4096. A Fourier transform of the intensity image will allow the spatial frequency content to be observed. Once again, the zero-padding of the intensity image in the image plane will only serve to 
provide enough samples for the spatial frequency harmonics. This can be clearly seen in Figure 27.

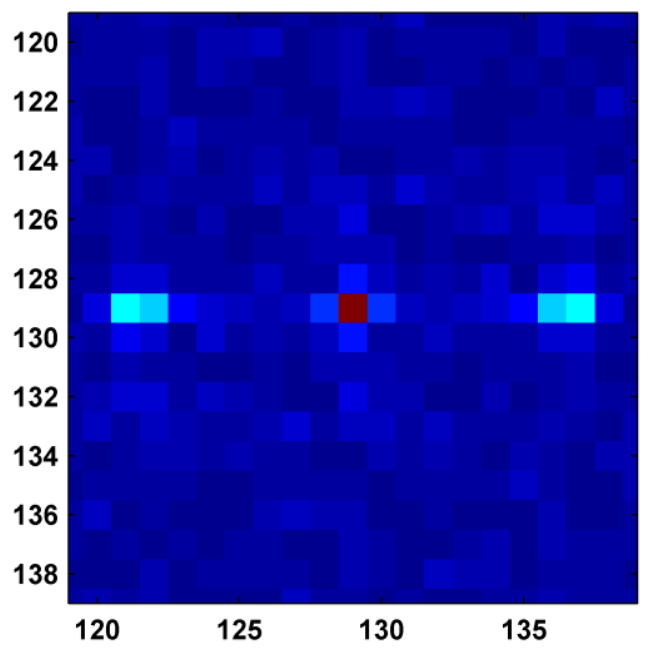

(a)

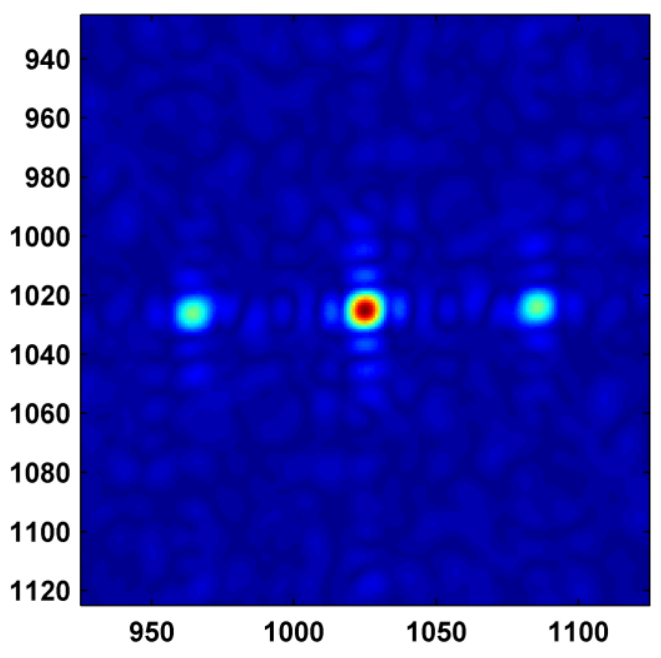

(b)

Figure 27: Shows the effects of proper sampling. (a) The spatial frequency content of an undersampled target image. (b) Spatial frequency content of a properly sampled target image.

The results found in Figure 27(a) depict the spatial frequency content of an intensity image that was not zero-padded. A Fourier transform was applied to the intensity image of a 512x512 image plane segment. The two brighter pixels on either side of the zero frequency, which are the first harmonic values, would not return the correct amplitude value due to the under-sampling issue. The result in Figure 27(b) depicts the spatial frequency content of a properly sampled image plane segment. As discussed previously, the intensity image segment was zero-padded to 4096x4096 which will provide enough samples under the first harmonic to obtain an accurate reading of the maximum amplitude.

A second process to ensure that the zero-padding step would provide enough samples was to isolate a specific first harmonic peak and then observe the cross-section to ensure that an absolute maximum could be obtained. In all cases, the first harmonic value 
directly to the right of the zero frequency was used in the analysis. The cross-section of the first harmonic value can be seen in Figure 28. This figure is in units of pixels and it can be seen from the figure that roughly 20 pixels are found underneath the first harmonic cross-section. The un-normalized amplitude value was found to be $8.9 \times 10^{13}$ and the overall uniform shape of the curve was found to have sufficient sampling.

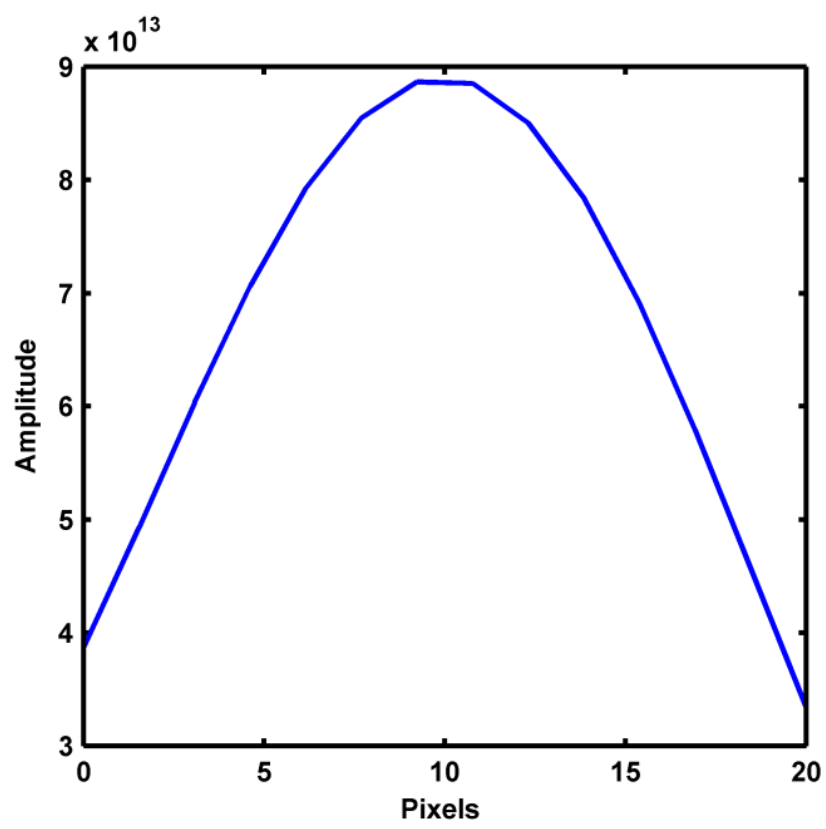

Figure 28: First harmonic cross-section.

Figure 29 displays a cross section plot through the spatial frequency content image in Figure 27b. The figure shows the zero frequency peak in the center of the plot, also known as DC level, along with two smaller peaks which are the first harmonic values. The cross section plot in Figure 29 was taken through one row of Figure 27(b), however, it should be noted that the most efficient method of finding the peak value was not directly obtained from this plot. The vertical bars from the up-sampled image in Figure 26 appear to be vertical, perpendicular with the horizontal axis which is not the situation. 


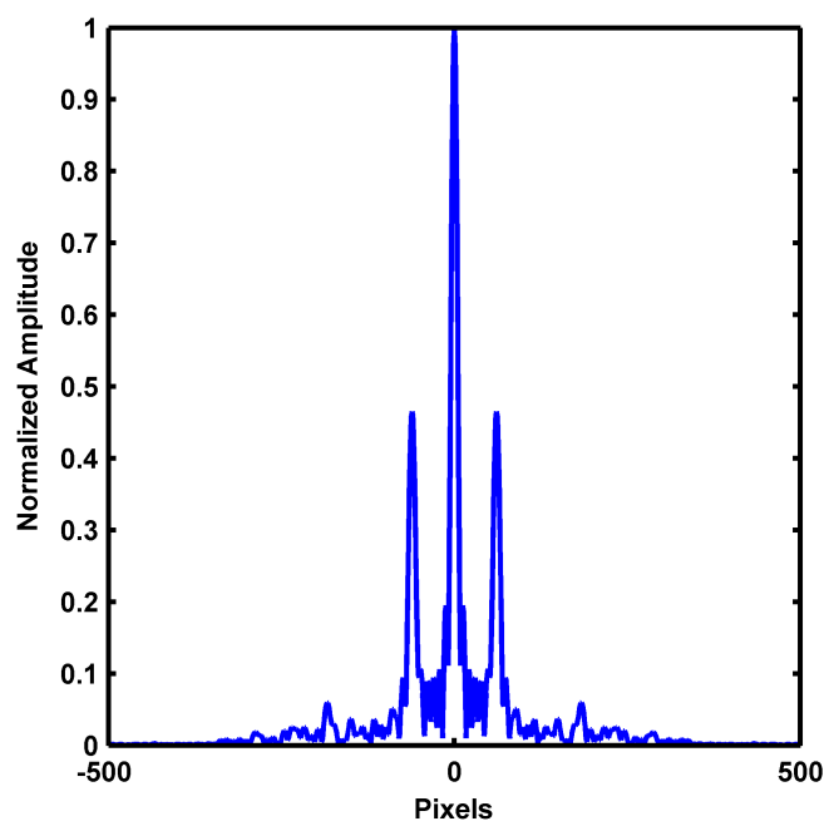

Figure 29: Cross section showing positive and negative first harmonic peaks.

Due to the fact the original target image was physically placed in the target plane, there may be, and in most cases will be, a slight amount of rotational tilt to the final upsampled image. Therefore, a Fourier transform of the slightly tilted up-sampled image will effectively cause the first harmonic peaks to rotate around the fundamental peak. Thus, the most efficient method of finding peak values was to physically crop out a $2 \mathrm{D}$ area containing the first harmonic and perform a peak value search.

The next step in the process, after obtaining first harmonic peak, value was to normalize this value to the zero frequency DC maximum and then to multiply the $\pi / 2$ scaling factor that was discussed in section 3.3. It should be note that the positive first harmonic peak to the right of the DC value was used for the MTF plots. The value was then plotted on the sub-aperture MTF line plot. In efforts to produce the sub-aperture MTF plot, the process outlined in this sub-section was repeated for Ronchi targets at $0.25,0.50,0.75,1.00$ and $1.25 \mathrm{cyc} / \mathrm{mm}$. 


\subsection{HAL Processing Steps}

In general terms, a holographic aperture ladar system makes use of a transmitter and a two dimensional receiver aperture in synchronous motion. The complex pupil plane field returning from the target is then coherently recorded, for example through conventional off-axis holography techniques, at a sequential collection of sub-aperture locations. Through application of the HAL transformation, a linear phase correction is then applied to each field segment according to the location of the transmitter at the instant the data was collected, after which each field segment is repositioned and placed in an extended coherent pupil plane array. After correcting for non-uniform phase aberrations, taking the two dimensional Fourier transform of the effective pupil plane field will cause an image of the target to be created that will have a higher longitudinal cross-range resolution than could be obtained from a single sub-aperture alone [2]. As might be imagined, though, in practice the data processing steps required are somewhat more involved. In this section the processing steps we have employed for examining our extended diffuse targets are described in detail, including the speckle registration and relative piston phase correction algorithms, and our method for generating the MTF of our laboratory based stripmap HAL system.

Figure 30 shows our HAL processing flow chart and outlines the sequence of steps taken to produce the MTF our system. The individual steps are shown in boxes sequentially numbered in the lower right hand corner. In most cases, the upper left hand corner of the boxes also contain either a $P$ or an $I$ to indicate that the processing step takes place in either pupil plane (i.e., the plane of the CCD sensor) or image plane, 
respectively. The dashed boxes indicate when a sub-pixel speckle field image registration algorithm was used [22].

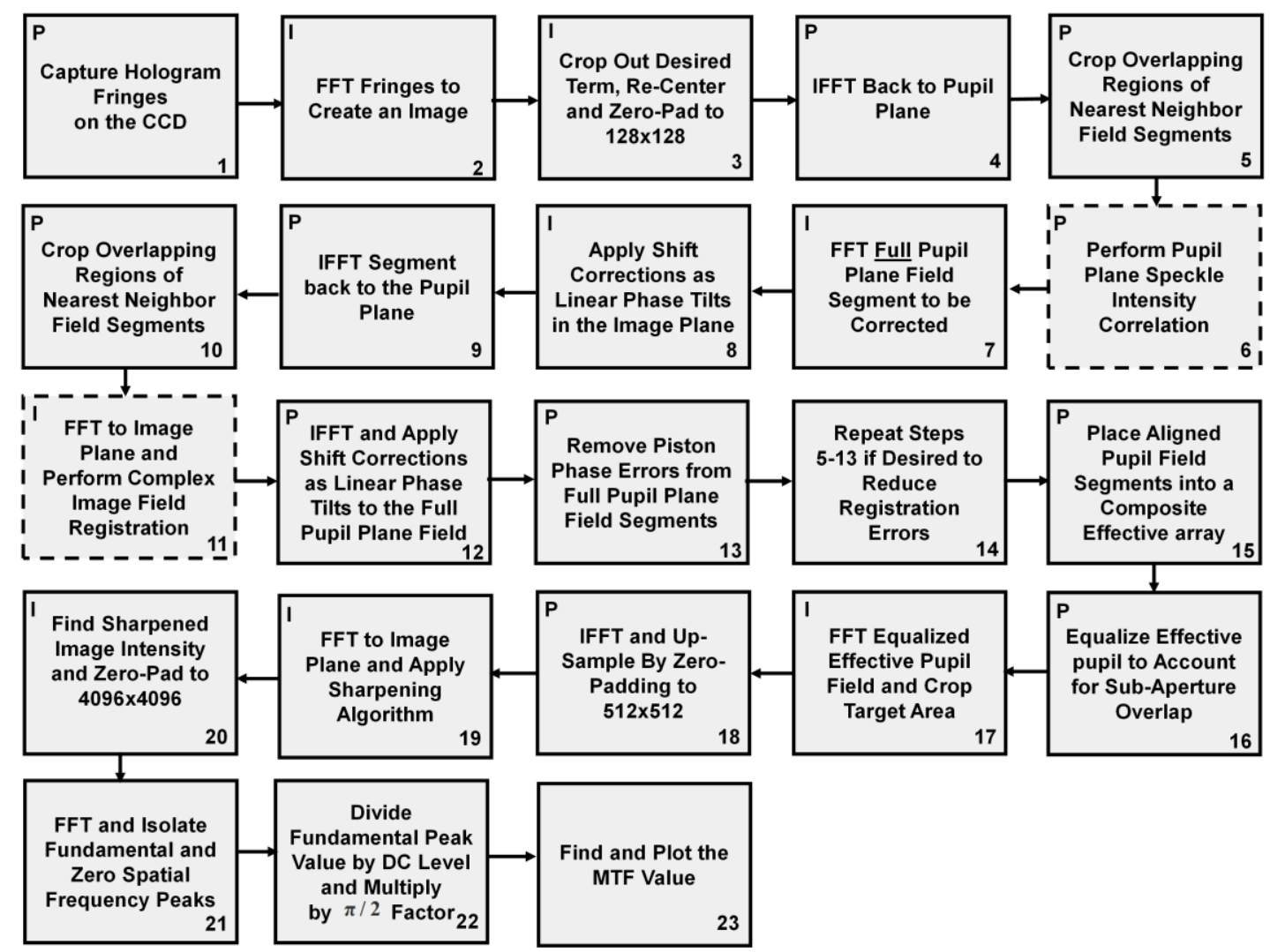

Figure 30: The HAL data processing method.

The first step is to capture fringes on the CCD sensor as the target is translated relative to the RX aperture. The field returning from the illuminated target is mixed with the $\mathrm{LO}$ beam on the $\mathrm{CCD}$, after which a fringe intensity pattern, or hologram, is recorded. An example of these fringes is shown in Figure 31 for our $0.25 \mathrm{cyc} / \mathrm{mm}$ target, where the horizontal and vertical axes are in units of pixels corresponding to the size of the digitally cropped CCD array. Also notice the somewhat discernible diagonal structure of this image. This structure arises due to the fact that the LO is tilted with respect to the CCD pupil plane surface normal. 


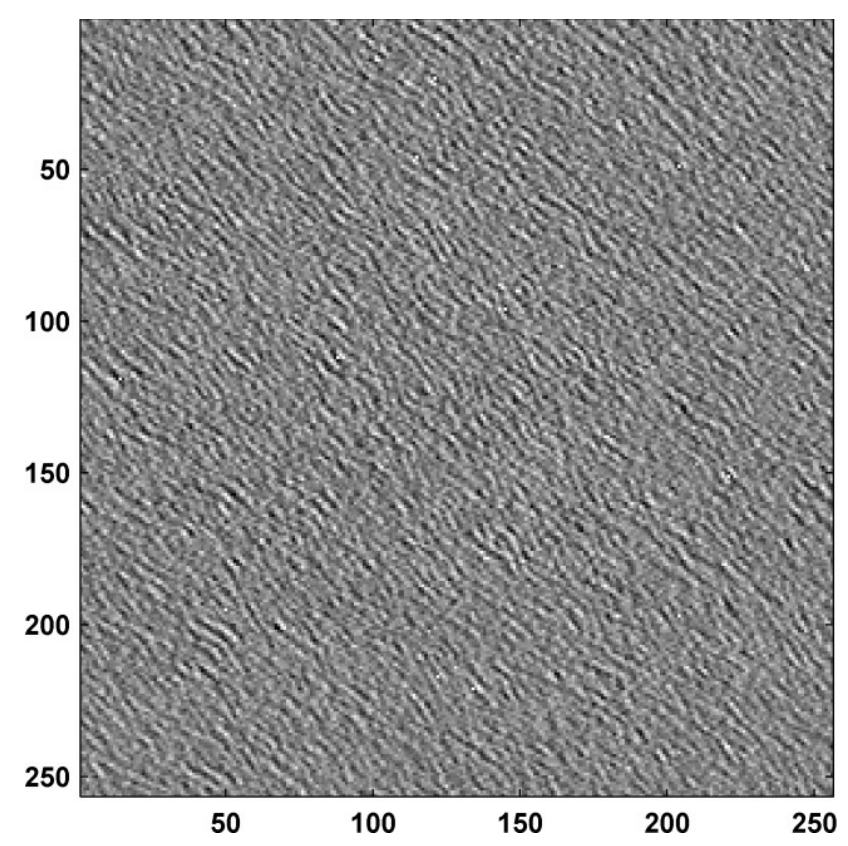

Figure 31: An example off-axis hologram of our $0.25 \mathrm{cyc} / \mathrm{mm}$ target containing a digitally cropped array of $256 \times 256$ pixels. The somewhat discernible diagonal structure of this image is due to the tilted LO beam.

The next step is to create an image of the target via Fourier transform. Due to the fact that the LO was placed in the plane of the target, the returning object and reference fields will have the same propagation induced phase curvature. The image term (upper right hand quadrant) and its conjugate will therefore both be in focus as shown, for example, in Figure 32.

Note that after taking the Fourier transform of the fringes obtained in the first step, a complex image field is created. Figure 32 shows only the modulus of this field. Moreover, it should be mentioned at this point that the tip/tilt and location of the LO aperture in the plane of the target was carefully adjusted during initial alignment to ensure that the image and conjugate terms fell in the geometric centers of their respective quadrants when the surface normals of the CCD and target were made coaxial. 


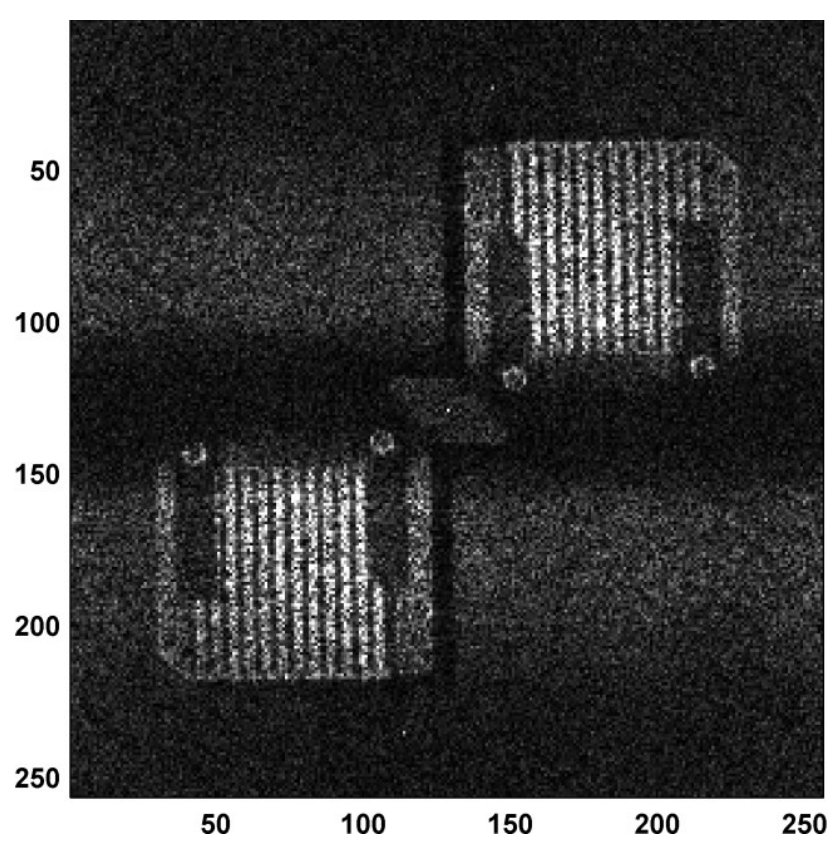

Figure 32: Modulus of the Fourier transform of the hologram shown in Figure 31. The 256x256 array contains two focused images of the target and the central $\mathrm{LO}$ autocorrelation term.

Due to the fact that the LO is largely planar across the CCD and much brighter than the returning target field, the autocorrelation of the reference beam is reduced to a single bright pixel in the center of Figure 32, whereas the autocorrelation of the target beam is not discernible at all.

As seen in Figure 32, the target image and its conjugate do not fill the quadrants in which they lie. Due to the fact that the illumination beam footprint was allowed to freely diffract in the cross-range dimension, the surrounding area in each respective quadrant has a considerable amount of unwanted background noise. The next step, then, is to tightly crop out the desired image term in order to eliminate both background noise and the LO autocorrelation term. As shown in Figure 33a, to preserve resolution, we then zero-pad the cropped image term back up to $128 \times 128$ pixels (i.e., the size of one quadrant), after which we inverse Fourier transform the data back to the pupil plane, Figure 33b. 

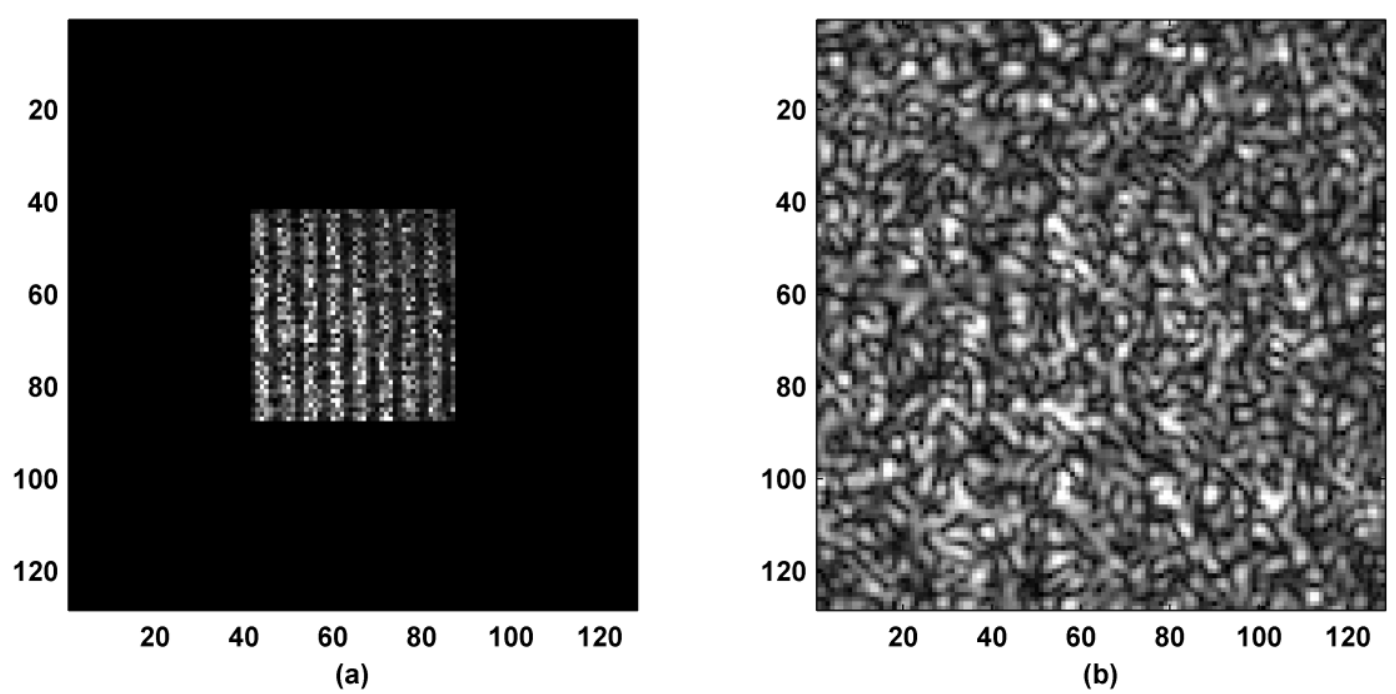

Figure 33: (a) The cropped and zero-padded image term of Figure 5 is (b) inverse Fourier transformed back to the pupil plane. Only the moduli of the complex fields are shown in both cases.

For each Ronchi ruling target the preceding steps are then repeated as the target is translated in discrete $2 \mathrm{~mm}$ steps across the synthetic aperture.

Recall that the synthetic aperture is the maximum distance the TX/RX combination, or the target, from a relative motion perspective, moves during the course of data collection. In our case we collected 12 frames of data for each Ronchi ruling, resulting in a synthetic aperture of $24 \mathrm{~mm}$ [4]. Also recall that if the interframe translation is exactly $D_{a p} / 2$, after HAL processing neighboring frames of data will be just contiguous. If, however, the interframe translation $\Delta_{x}$ is less than $D_{a p} / 2$, after HAL processing neighboring frames of data will overlap by $100\left(1-2 \Delta_{x} / D_{a p}\right)$ percent. In our case $\Delta_{x}=2 \mathrm{~mm}$ and Dap $=7.68$ $\mathrm{mm}$, yielding an interframe overlap of approximately $48 \%$, or about 61 pixels.

To begin the interframe registration process, the overlapping regions of neighboring field segments expected after HAL processing were first cropped. These were then converted to intensity after which they were spatially aligned to sub-pixel 
accuracy through application of a $\operatorname{Matlab}^{\circledR}$ based speckle correlation/registration algorithm. The field segment taken on one end of the synthetic aperture was taken as a reference and the second through $12^{\text {th }}$ field segments were aligned relative to the first. Collectively, this first speckle correlation and registration step is analogous to the repositioning step required by the stripmap HAL transformation process [2].

The registration algorithm used was the efficient subpixel image registration by cross-correlation m-file available for free download from The Mathworks, Inc.[23]. This algorithm is capable of performing a two dimensional registration to within a fraction of a pixel. The algorithm uses selective up-sampling by a matrix-multiply discrete Fourier transform in order to reduce computation time and memory requirements. An up-sampled cross-correlation between the two segments is performed around a small area around the peak. The location of the optimized correlation peak is then mapped back to the original coordinate system, with relative offsets recorded in units of pixels [22].

The relative offsets thus obtained are then applied to the pupil plane field segment of the data frame to be re-positioned. In order to keep the array sizes constant throughout the various processing steps this is accomplished by applying image plane linear phase tilts to the Fourier transform of the full pupil plane field segment to be re-positioned, according to the relationship

$$
F_{i}^{\prime}(m, n)=F_{i}(m, n) \exp \left(-j 2 \pi\left(\frac{m r_{o p}}{N_{r}}+\frac{n c_{o p}}{N_{c}}\right)\right)
$$

where $F_{i}(m, n)$ is the Fourier transform of the full field segment to be re-positioned, $m$ and $n$ are in terms of pixel variables, $r_{o p}$ and $c_{o p}$ are, respectively, the pupil plane row and column shift corrections are also in units of pixels, and $N_{r}$ and $N_{c}$ are, respectively, the 
row and column dimensions of the pupil array, also expressed in pixels. (In our case $N_{r}=$ $\left.N_{c}=128.\right)$ After the pixel shift corrections have been applied in the form of a linear phase tilt in the image plane, the complex image plane array $F_{i}^{\prime}(m, n)$ is then inverse Fourier transformed back to the pupil plane for further processing. Steps 5-9 of Figure 30 are then repeated until all the field segments have been properly aligned. Typical pixel value corrections for $r_{o p}$ and $c_{o p}$ usually range from fractional pixels to one or two pixels.

The next step in our data processing method is to correlate and align the phases in the overlapping regions of neighboring frames of pupil plane data. To accomplish this we once again employ the efficient subpixel image registration by cross-correlation algorithm, described above, as it is capable of processing both real and complex data. However, in this instance we use the registration algorithm to process complex data in the image plane. To begin we once again crop overlapping regions of neighboring pupil plane field segments, after which we Fourier transform the results. The registration algorithm is then used to determine the image plane row and column shift corrections required to maximize the cross-correlation peak. The pixel shift corrections are then applied to the full corresponding pupil plane fields in the form of linear phase modulation terms. That is, similar to equation (29), pixel shift corrections determined in the image plane are applied in the pupil plane according to

$$
g_{p}^{\prime}(m, n)=g_{p}(m, n) \exp \left(j 2 \pi\left(\frac{m r_{o i}}{N_{r}}+\frac{n c_{o i}}{N_{c}}\right)\right) \text {, }
$$

where $g_{p}(m, n)$ is the full pupil plane field segment whose phase we are aligning with its nearest neighbor, $m$ and $n$ are pixel variables, $r_{o i}$ and $c_{o i}$ are, respectively, the image plane row and column shift corrections in units of pixels, and where $N_{r}$ and $N_{c}$ are, respectively, 
the row and column dimensions of the pupil array, also expressed in pixels. (In our case $N_{r}=N_{c}=128$.) Collectively, steps $10-12$ of Figure 30 are analogous to applying the linear phase corrections called for through direct application of the stripmap HAL transformation [2].

Another phase correction that needs to be performed is to eliminate piston phase errors that tend to accumulate as the target and $\mathrm{TX} / \mathrm{RX}$ planes translate relative to one another. This is easily accomplished by subtracting the phase fields of overlapping portions of, now otherwise aligned, neighboring field segments. The resulting phase difference can then be removed from the full phase field of one of the field segments, but not both. At this point the image and pupil plane registration steps may be repeated if desired, to further refine the alignment of neighboring frames of complex pupil plane data. In practice we found this to be unnecessary, however. Due to the fact that the registration algorithm we have used is fast, efficient and provides precise sub-pixel accuracy, the residual alignment errors we observed when iteratively performing steps 513 of Figure 30 were found to be very small, if not negligible. As we will demonstrate in the next section, single step registration allowed us to obtain nearly diffraction limited results. With the preceding registration steps complete, the collection of pupil plane field segments are now digitally assembled into an extended, effective pupil plane array (ISR $x$ $\left.N_{c}\right)=861$ pixels wide, which for subsequent computational efficiency is further zero padded out to 1024 pixels, as shown in Figure 34. Recall from our earlier discussion that our nearest neighbor field segments overlap by $100\left(1-2 \Delta_{x} / D_{a p}\right)=48 \%$. As a result, as demonstrated in Figure 34a, the nominal composite field amplitudes in the overlapping regions are double that in the non-overlapping regions. 


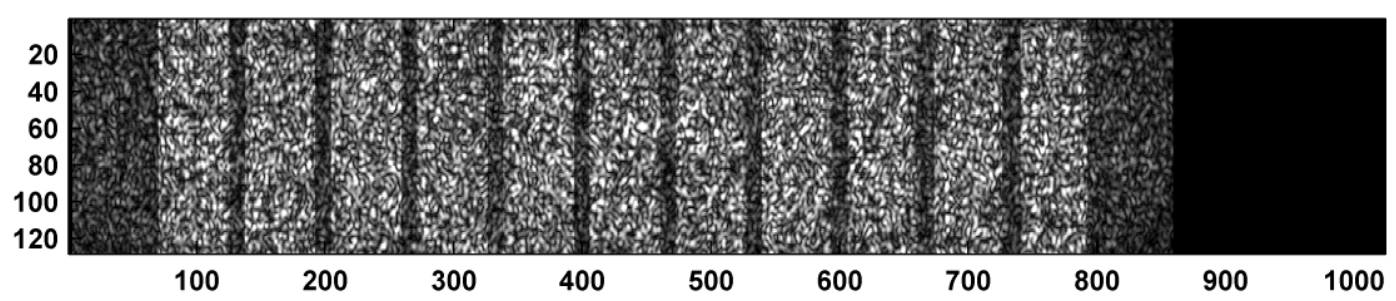

(a)

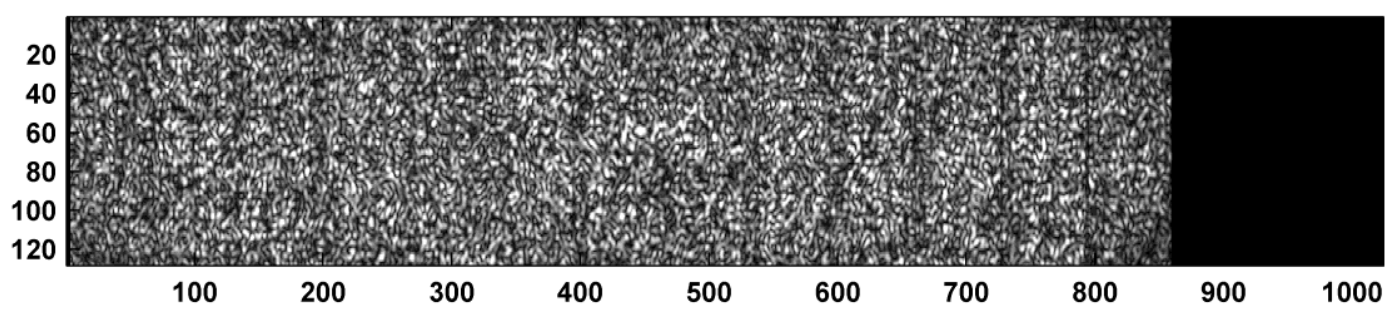

(b)

Figure 34: Modulus of the composite, effective pupil plane array (a) before amplitude equalization and (b) after amplitude equalization.

To correct this, a simple amplitude equalization mask is applied to the effective pupil plane array, yielding the nominally uniform result shown in Figure 34b.

The equalized effective pupil can then be Fourier transformed to produce an image with higher resolution which can be seen in Figure 35.

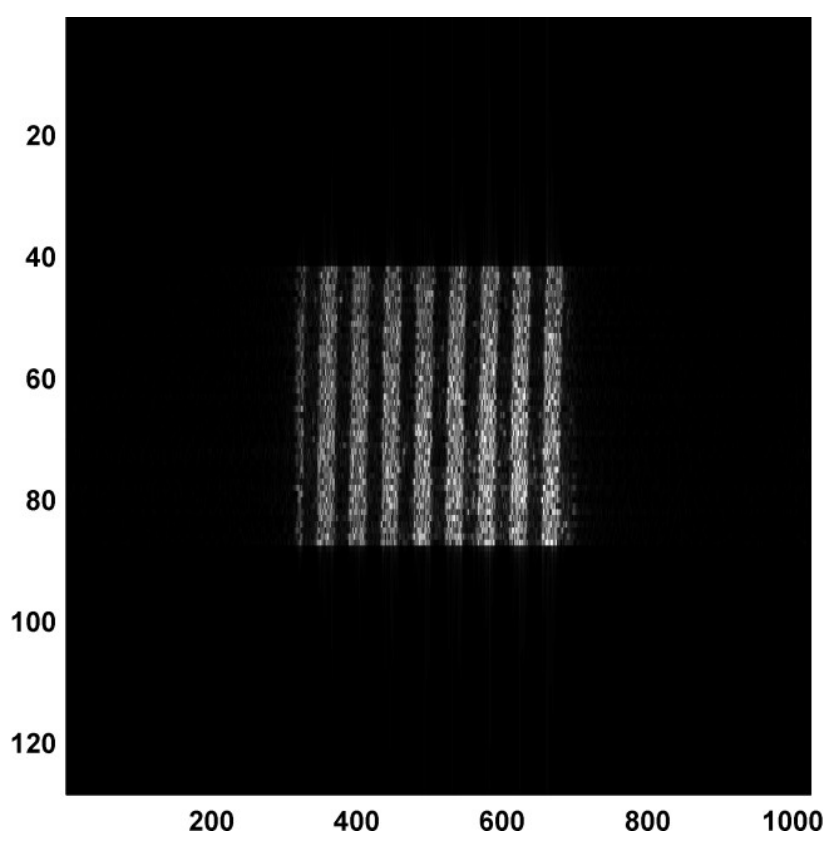

Figure 35: Fourier transform of the synthetic pupil resulting in an image plane segment. 
The Fourier transform of the effective pupil produces a higher resolution image that is centered in an array of zeros due to the fact that the pupil plane segments were upsampled in a previous step. The image is then cropped out of the array and inverse Fourier transformed back to the pupil plane where it is zero-padded to $512 \times 512$ then Fourier transformed to the image plane to produce an up-sampled synthetic image.

It should be noted that the master oscillator was physically placed, as close as possible, to the plane of the target object. However, any mismatch between the two will produce propagation induced phase errors due to range uncertainties. This will produce an overall defocus and will need to be corrected in efforts to produce the final synthetic image.

The image sharpening method we have employed seeks to minimize a real valued sharpness metric $S$ defined as

$$
S=\sum_{m=1}^{N_{s}} \sum_{n=1}^{N_{s}}\left|\mathfrak{J}\left\{g_{u}(m, n) \phi_{d}^{\beta}(m, n)\right\}\right|^{\gamma},
$$

where $g_{u}(m, n)$ is the zero-padded pupil plane field of the unsharpened image created subsequent to performing step 18 of Figure 30, $N_{s}$ is the number pixels (in our case $N_{s}=$ 512 ) in both the horizontal and vertical dimensions of the now square complex matrix, $m$ and $n$ are, as before, unitless row and column integer pixel variables, respectively, $\mathfrak{J}$ indicates the two dimensional Fourier transform operation, $\beta$ and $\gamma$ are parameters chosen to optimize image sharpness and where $\phi_{d}(m, n)$ is a general spherical phase function expressed as

$$
\phi_{d}(m, n)=\exp \left(\pi j \frac{\left(\left(2 m-N_{s}-1\right)^{2}+\left(2 n-N_{s}-1\right)^{2}\right)}{\left(N_{s}-1\right)^{2}}\right) \text {, }
$$


Notice that $\phi_{d}$ is centered on the pupil plane and that in the corners of the array (i.e., when in any combination the pixel variables $m$ and $n$ equal $N_{s}$ or 1) $\phi_{d}$ experiences $2 \pi$ radians, or one wave, of phase migration.

To sharpen the synthetic image and compensate for residual defocusing errors, $g_{u}(m, n)$ is first multiplied by $\phi_{d}$ raised to the $\beta$ power. An image is then formed via Fourier transform, after which the modulus of the image is raised to the $\gamma$ power. The result is then integrated in two dimensions to yield a value for sharpness metric $S$. (Note that if $\gamma=2$ it is the image intensity which in integrated in equation 13.) The effect of any residual defocusing phase error will, of course, be to blur the final synthetic image. This in turn results in bright image pixels being decreased in intensity towards the mean, while darker pixels will correspondingly tend to increase in intensity. The goal of the sharpness algorithm is then to effectively stretch the intensity histogram of the image by eliminating defocus phase errors, thereby producing better image contrast [24]. While the optimum value for $\beta$ is best determined iteratively, the optimum value for $\gamma$ is largely dependent upon the target. For high contrast targets such as our chrome-on-glass Ronchi rulings a $\gamma$ value somewhat less than unity is generally preferred [25]. As has been previously demonstrated, this has the effect of preferentially making dark pixels darker [24][25][26]. In particular, for our work we have found that setting $\gamma$ to 0.8 yields very good results [25].

In order to determine the optimum value of $\beta$ we used the fminbnd function of the Matlab $^{\circledR}$ Optimization Toolbox. To minimize $S$ we iteratively cycled through values of $\beta$, terminating the search when the absolute change in $S$ between iterations was found to be $10^{-4}$ or smaller. Though largely irrelevant to our final results, we note that at the point of 
convergence our unitless sharpness metric commonly had a value on the order of 1300 . We mention this only to offer a general sense of the precision to which we attempted to sharpen our final synthetic images. We also note that in the vast majority of cases the optimum $\beta$ values for our data were on the order of a modest -1.0 which can be seen in Table 1.

\begin{tabular}{ccc}
\hline Iteration & Optimization Variable $[\beta]$ & $\mathrm{S}_{\min }$ \\
\cline { 2 - 3 } 2 & -0.980902 & 1314.09 \\
\cline { 2 - 3 } 3 & -0.969098 & 1314.16 \\
4 & -0.988197 & 1314.05 \\
5 & -0.992705 & 1314.02 \\
6 & -0.995492 & 1314.01 \\
7 & -0.997214 & 1314 \\
8 & -0.998278 & 1313.99 \\
9 & -0.998936 & 1313.99 \\
10 & -0.999342 & 1313.98 \\
11 & -0.999593 & 1313.98 \\
12 & -0.999749 & 1313.98 \\
13 & -0.999845 & 1313.98 \\
14 & -0.999904 & 1313.98 \\
\hline
\end{tabular}

Table 1: Optimization data for the $6.00 \mathrm{cyc} / \mathrm{mm}$ target data set. The iterations are presented along with their respective optimization variable and $S_{\min }$ value.

While applying the sharpening algorithm to each specific spatial frequency target can be performed, our results showed that this process is not always needed. It should be noted again that each spatial frequency target was physically placed in the target plane in order to collect the multiple sets of data. The local oscillator exit aperture was also physically placed as close as possible to the plane of the target, however, there could be and in most cases will be a slight miss-match between these two planes which will ultimately lead to a de-focus phase error. However, due to the fact that the physical setup, (i.e. the target plane and LO) remain unchanged as different targets loaded, the defocus phase error will be consistent for each respective data set. The optimization 
results in Table 2 show that the optimum value for $\beta$ converges to -1 for the $1 \mathrm{cyc} / \mathrm{mm}$ target data set. Therefore, it can be concluded that for laboratory scenarios where the target plane and LO exit aperture plane remain unchanged, the sharpening algorithm can be performed once and the $\beta$ value can be used for resulting target data sets. It is also suggested that the sharpening algorithm be performed on a higher frequency target due to the fact that the image will be more sensitive to defocus.

\begin{tabular}{ccc}
\hline Iteration & Optimization Variable $[\beta]$ & $S_{\min }$ \\
\cline { 2 - 3 } 3 & -0.961803 & 1190.78 \\
3 & -0.938197 & 1191.45 \\
4 & -0.976393 & 1190.37 \\
5 & -0.985410 & 1190.12 \\
6 & -0.990983 & 1189.96 \\
7 & -0.994427 & 1189.87 \\
8 & -0.996556 & 1189.81 \\
9 & -0.997871 & 1189.77 \\
10 & -0.998684 & 1189.75 \\
11 & -0.999187 & 1189.73 \\
12 & -0.999498 & 1189.73 \\
13 & -0.999689 & 1189.72 \\
14 & -0.999808 & 1189.72 \\
15 & -0.999881 & 1189.72 \\
16 & -0.999927 & 1189.71 \\
\hline
\end{tabular}

Table 2: Optimization data for the $1.00 \mathrm{cyc} / \mathrm{mm}$ target data set. The iterations are presented along with their respective optimization variable and $S_{\min }$ value.

While an optimum value for $\beta$ will be highly dependent upon the experimental arrangement, our $\beta$ values indicate that our MO exit aperture and targets were very nearly co-planar, as we had intended for them to be.

After the appropriate $\beta$ value has been determined for a particular image, it is then applied to the corresponding synthetic pupil plane field according to the relationship

$$
g_{s}(m, n)=g_{u}(m, n) \phi_{d}^{\beta}(m, n)
$$


Taking the Fourier transform (i.e., step 19 of Figure 30) of the now focus corrected field $g_{s}(m, n)$ produces an optimally sharpened, fully HAL processed, final synthetic image. It is the sharpened images of our targets that are then used in steps 20 and following of the flowchart in Figure 30.

The up-sampled, sharpened synthetic image for the $0.25 \mathrm{cyc} / \mathrm{mm}$ target can be seen in Figure 36 along with a side by side comparison of a sub-aperture image and HAL processed synthetic image in Figure 37. The final HAL processed synthetic image shows a unmistakable increase in resolution. It should also be noted that the sharpening algorithm was applied to the sub-aperture image as well.

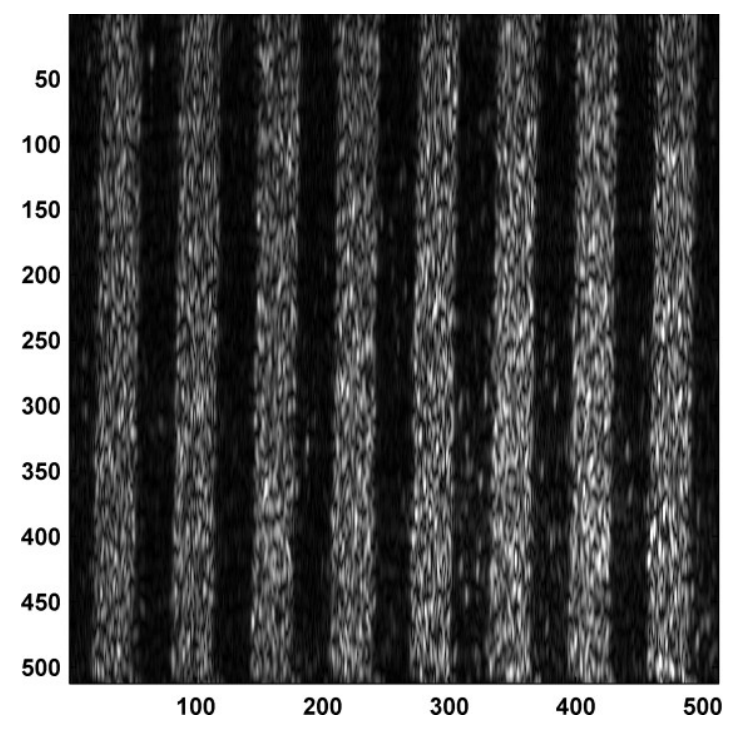

Figure 36: The final HAL processed synthetic image. The image was up-sampled to $512 \times 512$ by cropping out the target area from Figure 35, IFFT to the pupil plane, zero-padding to $512 \times 512$ and FFT back to the image plane.

The respective images of Figure 37 depict the target object area of the Ronchi target that was cropped due to the fastening apparatus. The approximate area of the 2" target that was used was a $32 \mathrm{~mm} \times 32 \mathrm{~mm}$ sub-section where a visual increase in resolution can be seen between the two images. 


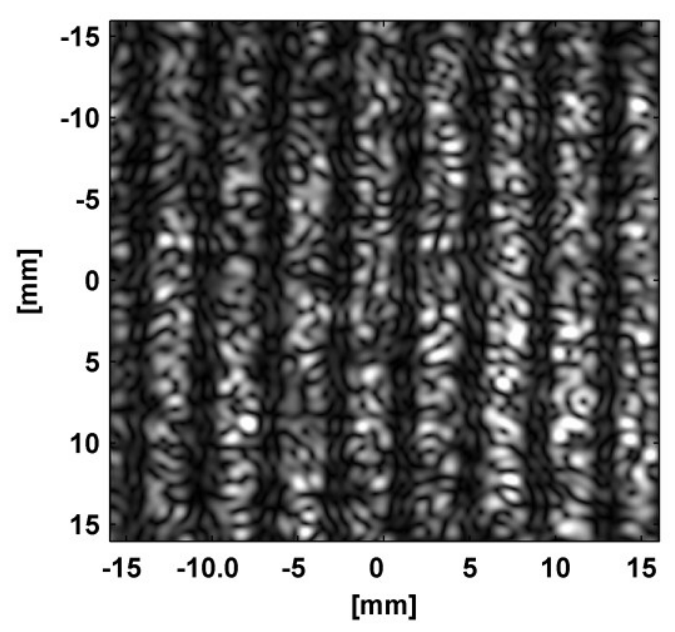

(a)

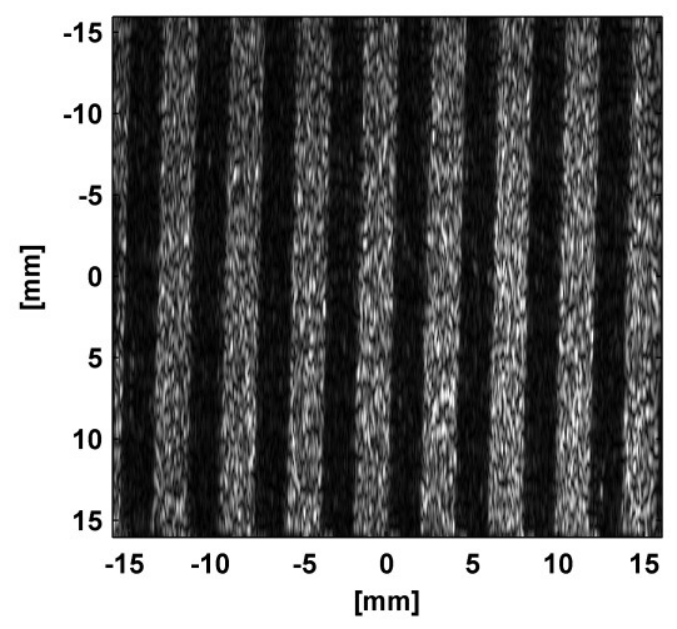

(b)

Figure 37: Side by side visual comparison of a $0.25 \mathrm{cyc} / \mathrm{mm}$ (a) sub-aperture image, and (b) a HAL processed synthetic image.

However, the $0.25 \mathrm{cyc} / \mathrm{mm}$ target is well below the sub-aperture frequency cutoff so that discernible features can be seen. Figure 38 depicts a side by side comparison of a 1 cyc/mm target where Figure 38a displays a 512x512 sub-aperture image corrupted by speckle. Figure 38 shows the 512x512 HAL processed image where the discernible bars of the single frequency Ronchi target can be seen.

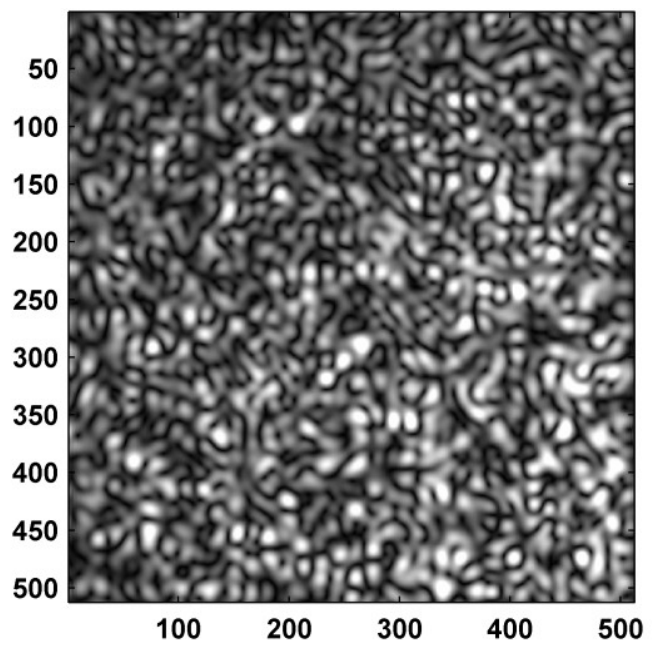

(a)

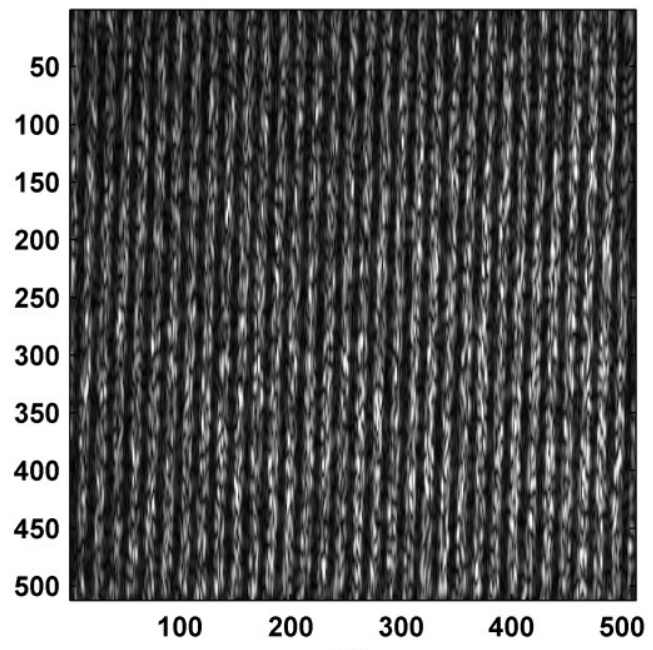

(b)

Figure 38: Side by side visual comparison of, (a) a $1 \mathrm{cyc} / \mathrm{mm}$ sub-aperture image, and (b) HAL processed synthetic image. 
The next step was to convert the complex image into an intensity image in order to gain access to the spatial frequency content of the target. The image was also zeropadded to $4096 \times 4096$ in efforts to provide enough samples under the spatial frequency harmonics.

One of the limiting factors that occur with coherent illumination is the element of speckle [11], [12], [27]. The chrome bars which were on one side of the Ronchi ruling slide were placed against a diffuse backing. The rough surface of the diffuse backing would then cause the speckle phenomena to occur. Thus, speckle limits the single subaperture resolution as well as the HAL processed image [27]. In efforts to reduce this limiting factor and achieve almost diffraction limited performance, a speckle average technique would need to be implemented. The two dimensional Fourier transform implemented by Matlab ${ }^{\circledR}$ is equivalent to performing the discrete Fourier transform of the column dimension of the array followed by a second discrete Fourier transform in the row dimension. The two dimensional Fourier transform is applied at the end of the processing steps, more specifically in step 21 of Figure 30, to produce the spatial frequency content and effectively performs a speckle average final HAL processed synthetic image.

A Fourier transform of the zero-padded, intensity image will produce Figure 39 which shows the spatial frequency content harmonics. The image has been cropped to $512 \times 512$ so that the DC point and harmonics are visible.

The first harmonic is the point of interest and is cropped out and the maximum value is found. The maximum value is then normalized by the maximum value of the DC point and is the multiplied by the $\pi / 2$ factor as discussed in Chapter 3 . 


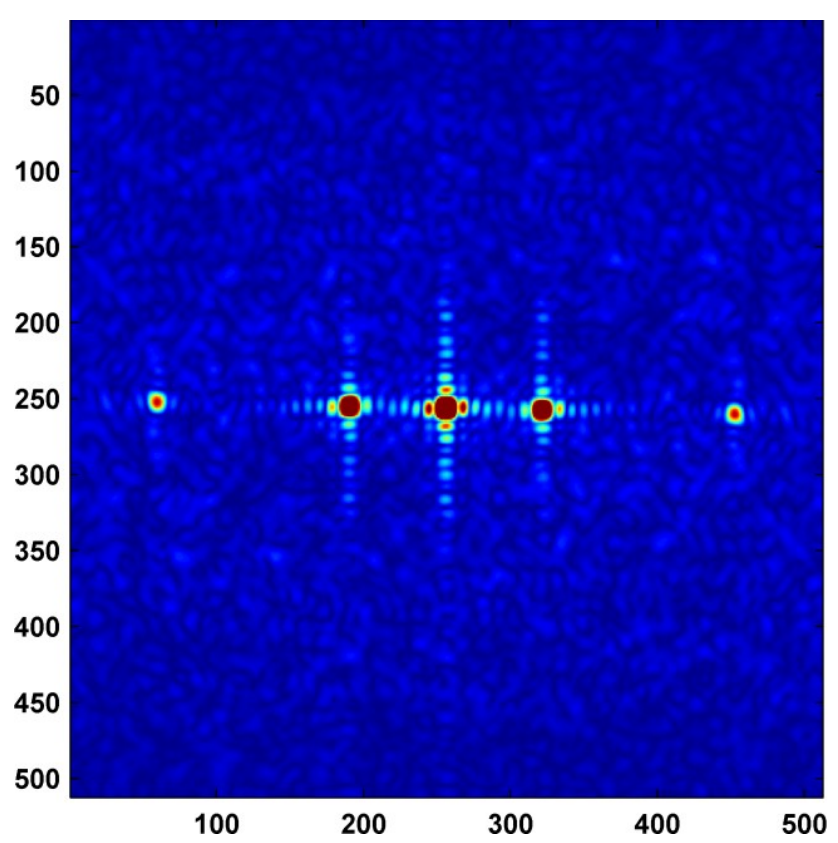

Figure 39: The spatial frequency content of the HAL processed synthetic image. The final synthetic image was converted to intensity, zero-padded to 4096x4096 and Fourier transformed to show the spatial frequency harmonics.

The value that results from this process will then be plotted on the MTF graph. The example in Figure 39 was only one of nine targets that were processed. For all of the targets, the HAL processing method was applied, the first harmonic value was selected and maximum value attained. This point was then scaled and plotted to produce a HAL processed MTF line.

The results that were obtained from the multiple data sets used in the construction of the HAL processed MTF can be found below in Table 3. In all, five data sets were obtained, each containing $N=12$ frames for each respective Ronchi ruling target in efforts to show the repeatability of the process. The mean data values were plotted MTF graph along with the standard deviations of the data to produce an error bar plot. Table 4 contains the sub-aperture data that was used in the construction of the sub-aperture MTF 
plot. Six independent frames of data were used and the mean value plotted for the MTF graph in efforts to show the repeatability of the process.

\begin{tabular}{|c|c|c|c|c|c|c|c|}
\hline \multirow[t]{2}{*}{$\begin{array}{c}\text { Target } \\
\text { [cyc/mm] }\end{array}$} & \multicolumn{5}{|c|}{ Data Set } & \multirow[t]{2}{*}{$\begin{array}{c}\text { Mean } \\
{[\mu]}\end{array}$} & \multirow{2}{*}{$\begin{array}{c}\text { Standard } \\
\text { Deviation } \\
{[\sigma]}\end{array}$} \\
\hline & 1 & 2 & 3 & 4 & 5 & & \\
\hline 0.25 & 0.9248 & 0.9162 & 0.9233 & 0.9207 & 0.9216 & 0.9213 & 0.0033 \\
\hline 0.50 & 0.8958 & 0.8918 & 0.9202 & 0.9224 & 0.9213 & 0.9103 & 0.0151 \\
\hline 0.75 & 0.8578 & 0.8602 & 0.8677 & 0.8657 & 0.8651 & 0.8633 & 0.0041 \\
\hline 1.00 & 0.8255 & 0.8314 & 0.8167 & 0.8122 & 0.8168 & 0.8205 & 0.0078 \\
\hline 1.25 & 0.8020 & 0.8041 & 0.8233 & 0.7957 & 0.8015 & 0.8053 & 0.0105 \\
\hline 2.00 & 0.7214 & 0.6883 & 0.6656 & 0.6490 & 0.6589 & 0.6766 & 0.0289 \\
\hline 4.00 & 0.5107 & 0.4588 & 0.4503 & 0.4468 & 0.4615 & 0.4656 & 0.0259 \\
\hline 6.00 & 0.2563 & 0.2387 & 0.2598 & 0.2726 & 0.2389 & 0.2533 & 0.0145 \\
\hline 8.00 & 0.0791 & 0.0646 & 0.0627 & 0.0654 & 0.0654 & 0.0674 & 0.0066 \\
\hline
\end{tabular}

Table 3: HAL processed data for the multiple targets used in the construction of the HAL processed MTF. The values listed for each data set were obtained using the HAL processing algorithm in Figure 30 and have the $\pi / 2$ scaling factor applied to the data.

\begin{tabular}{|c|c|c|c|c|c|c|c|c|}
\hline \multirow[t]{2}{*}{$\begin{array}{c}\text { Target } \\
{[\text { cyc/mm] }}\end{array}$} & \multicolumn{6}{|c|}{ Data Set } & \multirow{2}{*}{$\begin{array}{l}\text { Mean } \\
{[\mu]}\end{array}$} & \multirow{2}{*}{$\begin{array}{c}\text { Standard } \\
\text { Deviation } \\
{[\sigma]}\end{array}$} \\
\hline & 1 & 2 & 3 & 4 & 5 & 6 & & \\
\hline 0.25 & 0.7763 & 0.7810 & 0.7725 & 0.7921 & 0.8008 & 0.7892 & 0.7853 & 0.0106 \\
\hline 0.50 & 0.6090 & 0.6458 & 0.6058 & 0.5993 & 0.5870 & 0.6232 & 0.6117 & 0.0205 \\
\hline 0.75 & 0.4023 & 0.4294 & 0.4258 & 0.4175 & 0.4185 & 0.4199 & 0.4189 & 0.0094 \\
\hline 1.00 & 0.3778 & 0.2488 & 0.2381 & 0.2378 & 0.2450 & 0.2494 & 0.2435 & 0.0051 \\
\hline 1.25 & 0.0776 & 0.0609 & 0.0766 & 0.0731 & 0.0711 & 0.0764 & 0.0726 & 0.0062 \\
\hline
\end{tabular}

Table 4: Sub-aperture data for the multiple targets used in the construction of the sub-aperture processed MTF. The values listed for each data set were obtained using the steps found in Figure 24 and have the $\pi / 2$ scaling factor applied to the data. 


\section{CHAPTER 6}

\section{HAL PROCESSING RESULTS}

The results that were obtained in the lab and processed were divided into two sections in this chapter. First, a qualitative analysis was provided that included both sub-aperture final images along with a HAL processed synthetic images for a direct comparison. The nine figures that were formed show a substantial resolution enhancement, from that of the sub-aperture image, as a direct result from stitching together multiple apertures using the HAL processing method. It is clear to see that for some targets it is hard to determine if bars are able to be resolved.

For the results in section 6.2, a graphical representation in the form of an MTF was used to provide a quantitative measurement of the resolution enhancement by using the HAL processing method. In this section, a sub-aperture MTF was formed using the multiple single frequency Ronchi ruling targets. This MTF plot defined a diffraction limited cut-off frequency that was based on the system parameters. Then by using the techniques describe in HAL processing method, a synthetic image MTF plot was formed. Using the sub-aperture cut-off frequency, a direct comparison of resolution enhancement

could easily be seen. Multiple MTF plots were also generated that used a variety of effective aperture sizes based on the number of sub-apertures that were used. In total, 
three different MTF plots were formed for effective aperture sizes using $N=12,8$ and 5 sub-aperture frames.

\subsection{Qualitative Analysis}

The results shown in Figure 40-Figure 48 show a $512 \times 512$ sub-aperture image of the respective target next to a $512 \times 512 \mathrm{HAL}$ processed synthetic image. The theoretical sub-aperture cut-off was found to be $1.38 \mathrm{cyc} / \mathrm{mm}$ while the HAL effective aperture produced a cut-off of $9.29 \mathrm{cyc} / \mathrm{mm}$.

From the pictures shown below, in Figure 40(a) the vertical bars of the sub-aperture image are still quite visible; however, speckle has reduced the overall image quality. Figure 40(b) shows a vast improvement in visual resolution.

For the $0.75 \mathrm{cyc} / \mathrm{mm}$ sub-aperture image shown in Figure 42(a), speckle has degraded the image and the vertical bars have become difficult to distinguish. However, the HAL processed synthetic image shows a visual increase in resolution and a reduction in speckle size.

It can be seen from the multiple figures below that after 1 cyc $/ \mathrm{mm}$, the vertical bars of the sub-aperture images can no longer be resolved. This is due to the fact that as the spatial frequency target approaches the sub-aperture frequency cut-off, speckle tends to corrupt the image quality. In contrast, the HAL processed synthetic image shows a visual improvement in image resolution. This is highly apparent in for synthetic images of spatial frequency targets $0.25,0.50,0.75,1.00,1.25,2.00$ and $4 \mathrm{cyc} / \mathrm{mm}$. However, the 6 and $8 \mathrm{cyc} / \mathrm{mm}$ synthetic images are approaching the spatial frequency cut-off of $9.29 \mathrm{cyc} / \mathrm{mm}$ and it is difficult to resolve bars. The zoomed images of the respective images do show a vertical structure and a horizontal compression of speckle. 


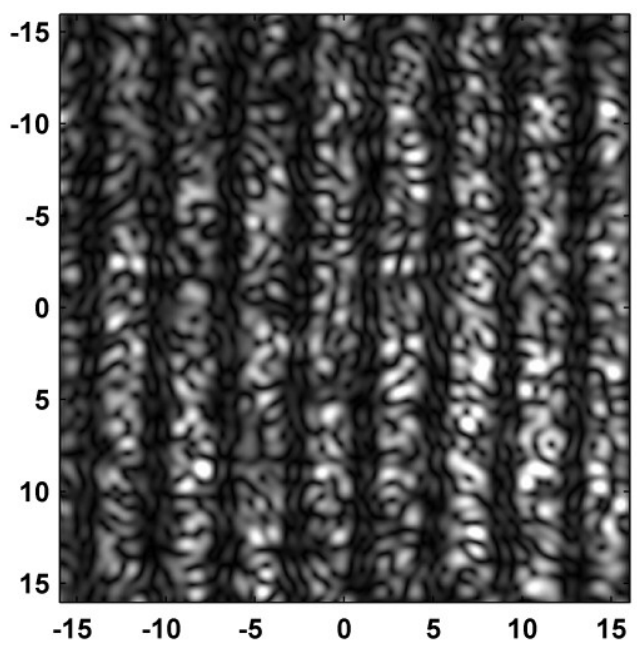

(a)

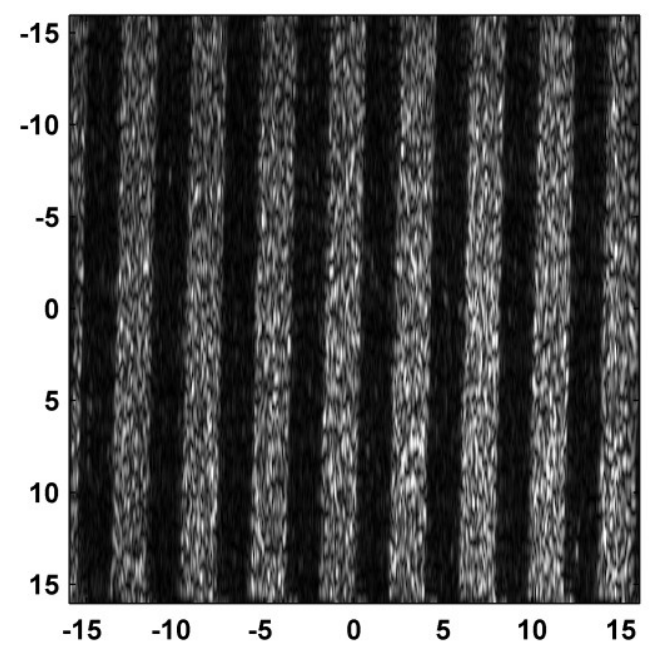

(b)

Figure 40: Side by side visual comparison of a, (a) $0.25 \mathrm{cyc} / \mathrm{mm}$ sub-aperture image and (b) HAL processed synthetic image.

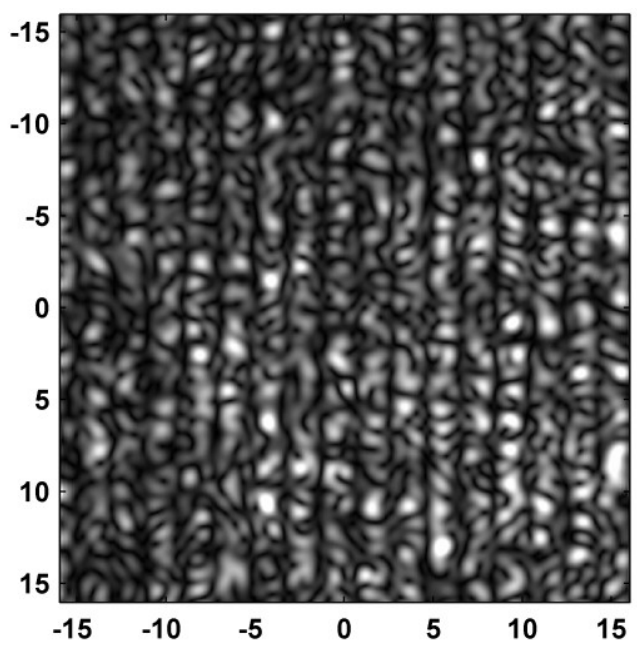

(a)

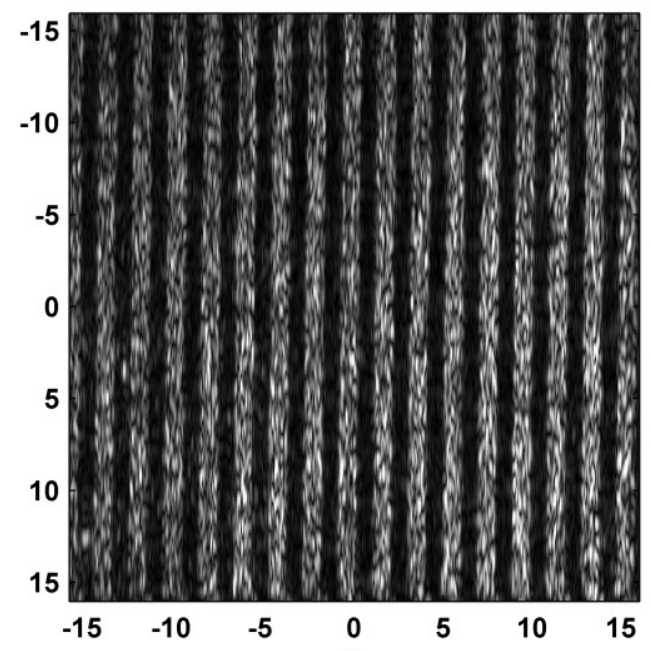

(b)

Figure 41: Side by side visual comparison of a, (a) $0.50 \mathrm{cyc} / \mathrm{mm}$ sub-aperture image and (b) HAL processed synthetic image. 

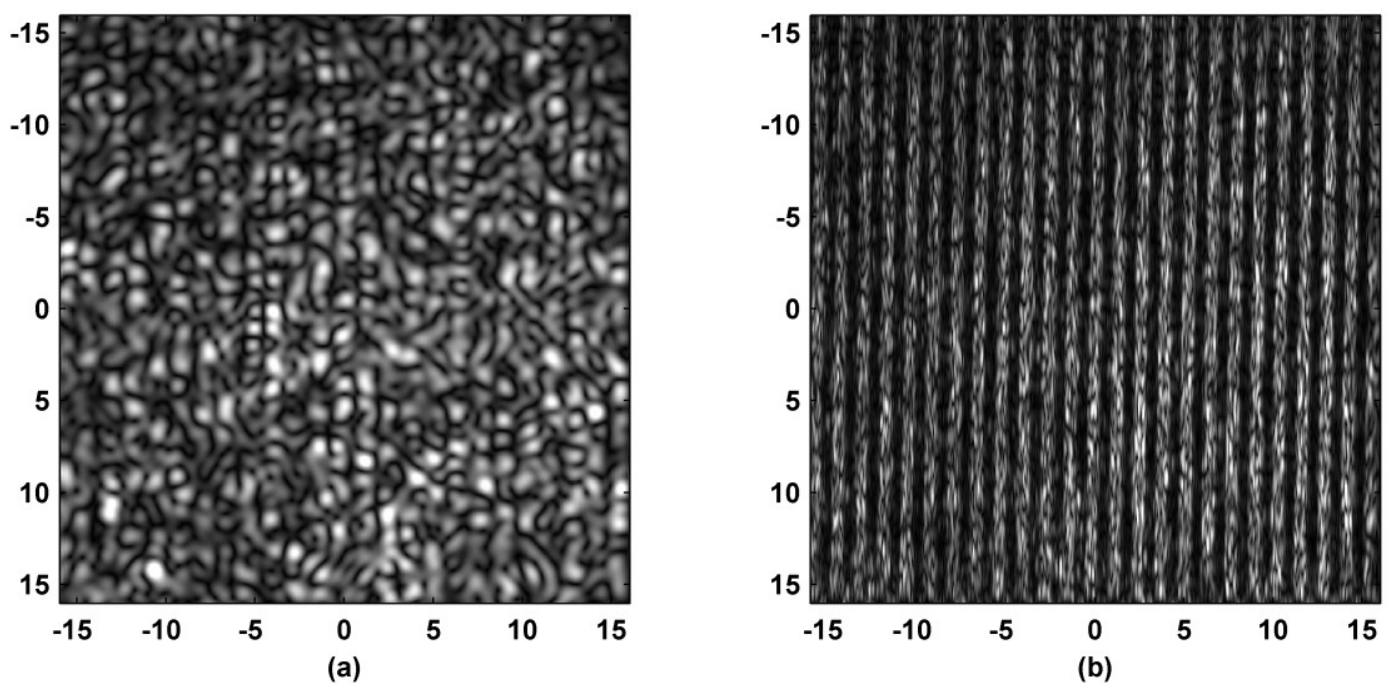

Figure 42: Side by side visual comparison of a, (a) $0.75 \mathrm{cyc} / \mathrm{mm}$ sub-aperture image and (b) HAL processed synthetic image.

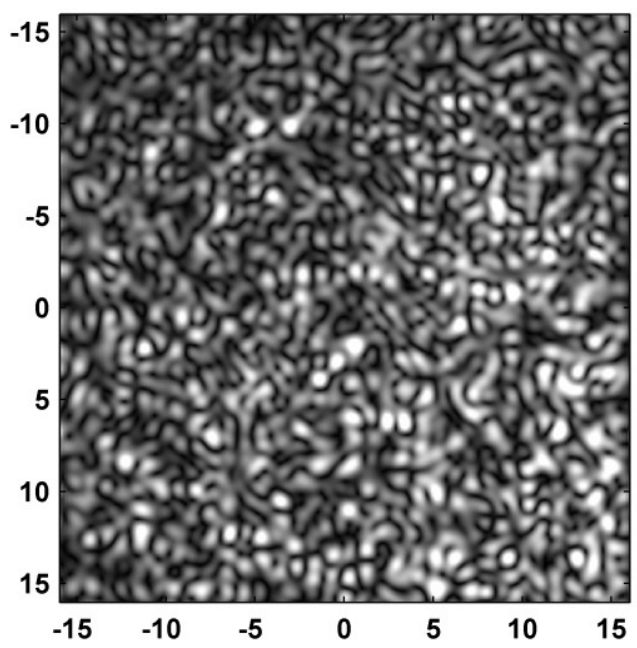

(a)

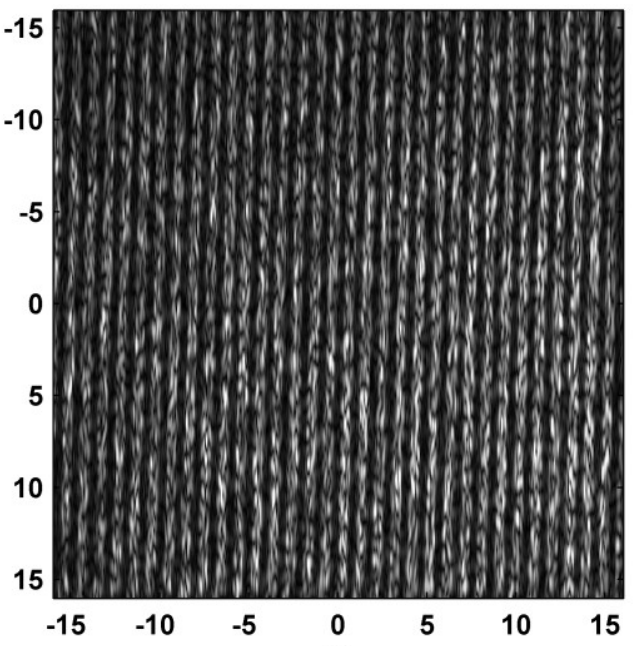

(b)

Figure 43: Side by side visual comparison of a, (a) $1 \mathrm{cyc} / \mathrm{mm}$ sub-aperture image and (b) HAL processed synthetic image. 


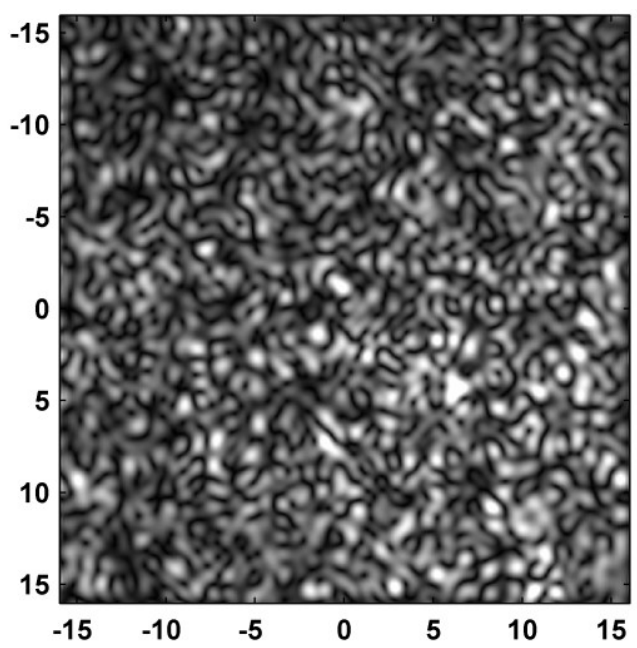

(a)

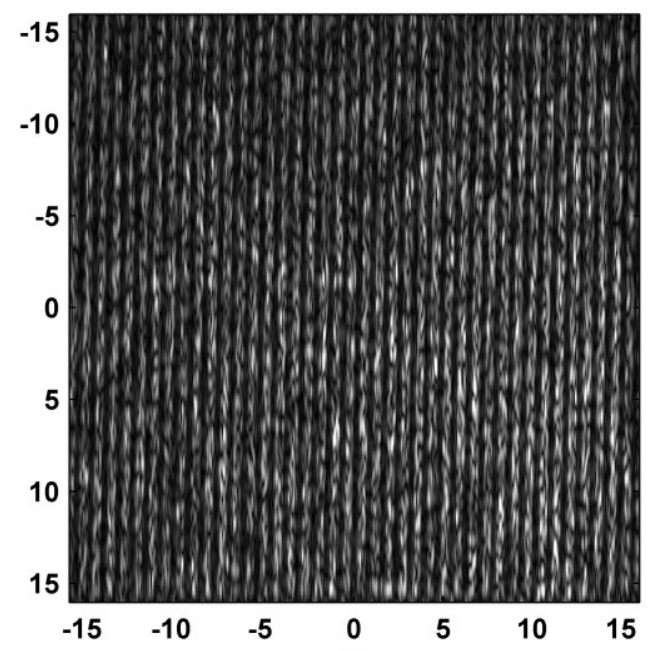

(b)

Figure 44: Side by side visual comparison of a, (a) $1.25 \mathrm{cyc} / \mathrm{mm}$ sub-aperture image and (b) HAL processed synthetic image.

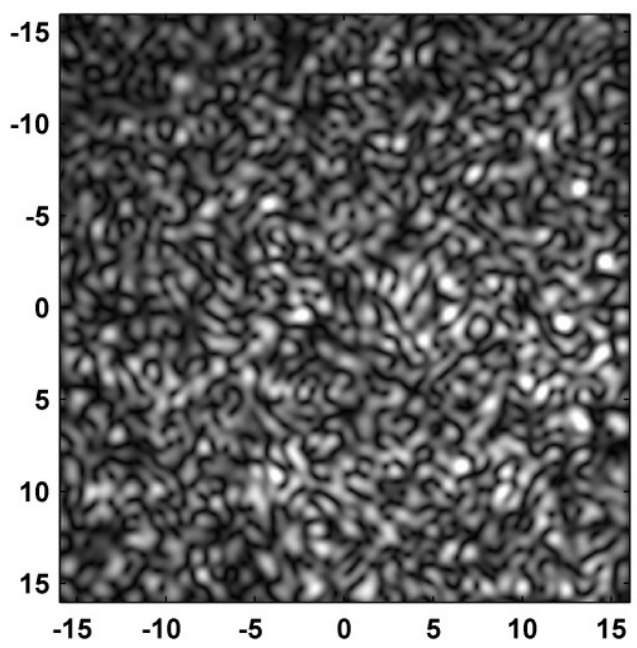

(a)

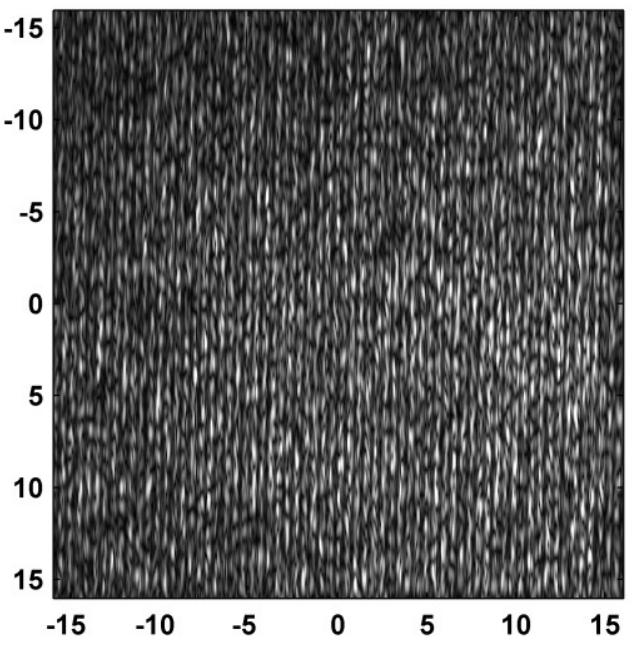

(b)

Figure 45: Side by side visual comparison of a, (a) $2 \mathrm{cyc} / \mathrm{mm}$ sub-aperture image and (b) HAL processed synthetic image. 


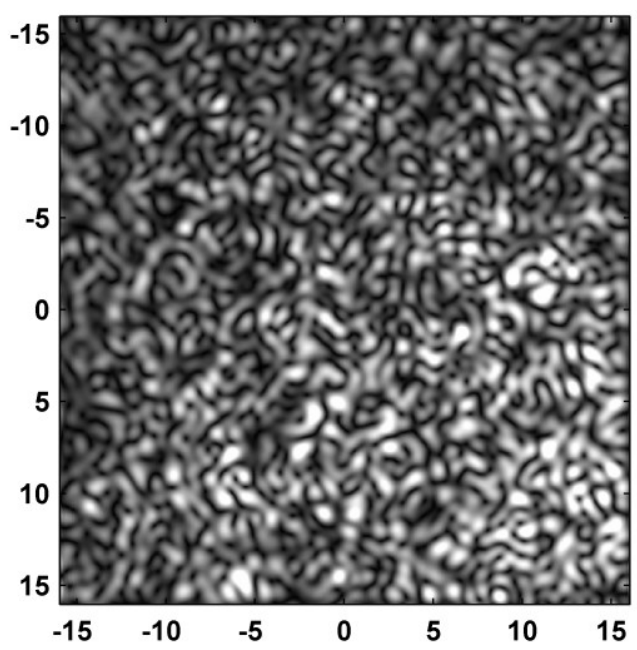

(a)

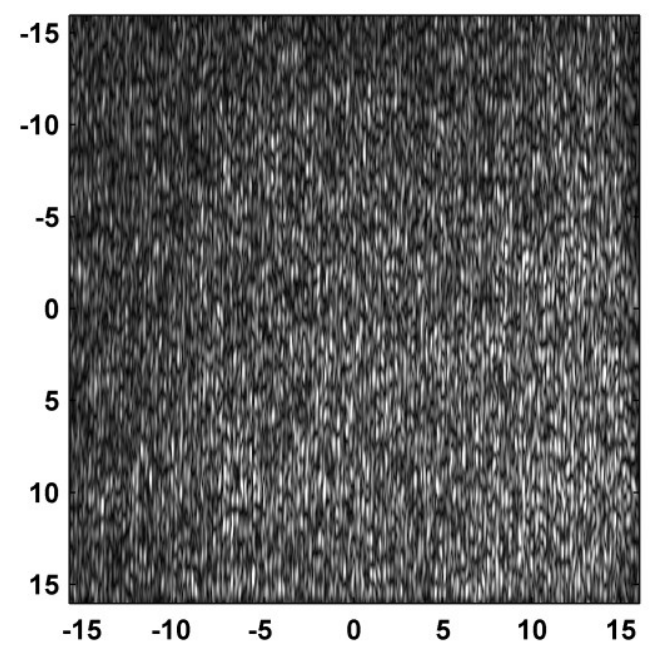

(b)

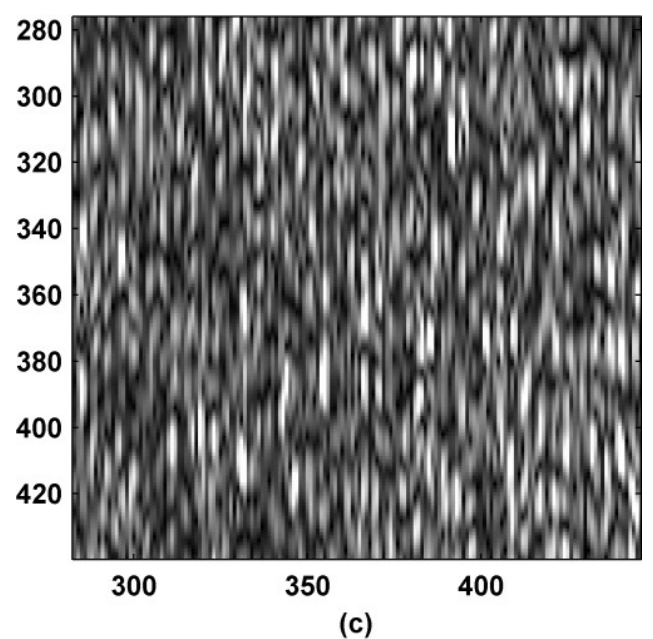

Figure 46: Side by side visual comparison of a, (a) $4 \mathrm{cyc} / \mathrm{mm}$ sub-aperture image and (b) HAL processed synthetic image and (c) a zoomed region of the HAL processed synthetic image. The vertical structure of the synthetic image is evident in the zoomed image. 


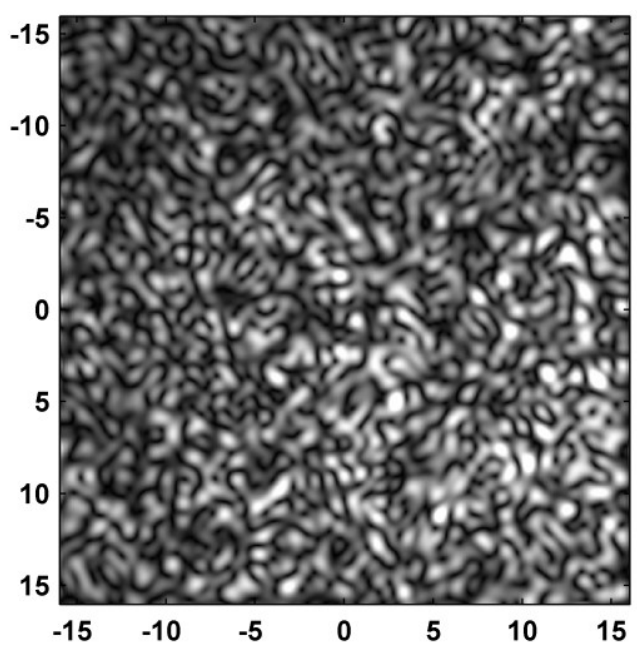

(a)

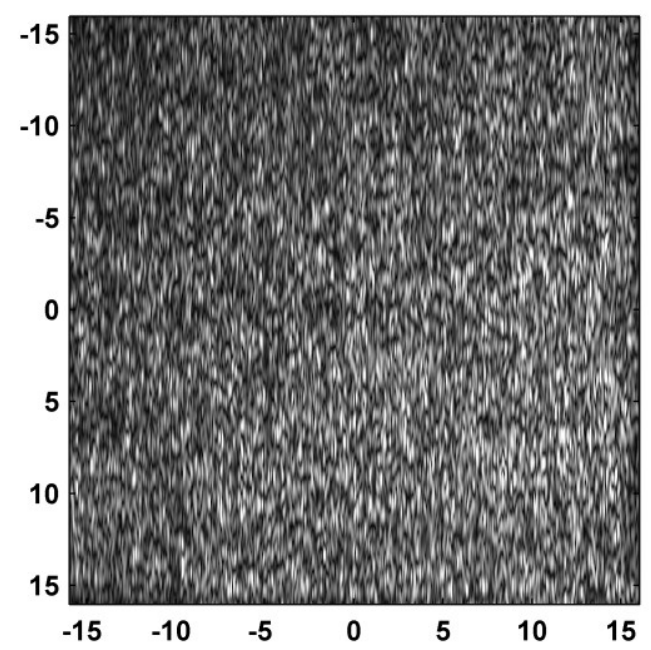

(b)

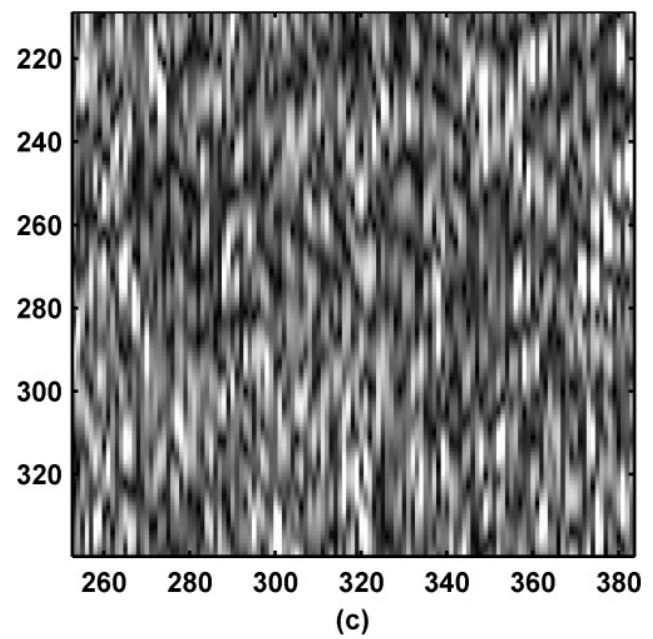

Figure 47: Side by side visual comparison of a, (a) $6 \mathrm{cyc} / \mathrm{mm}$ sub-aperture image and (b) HAL processed synthetic image and (c) a zoomed region of the HAL processed synthetic image. The vertical structure of the synthetic image is evident in the zoomed image. 


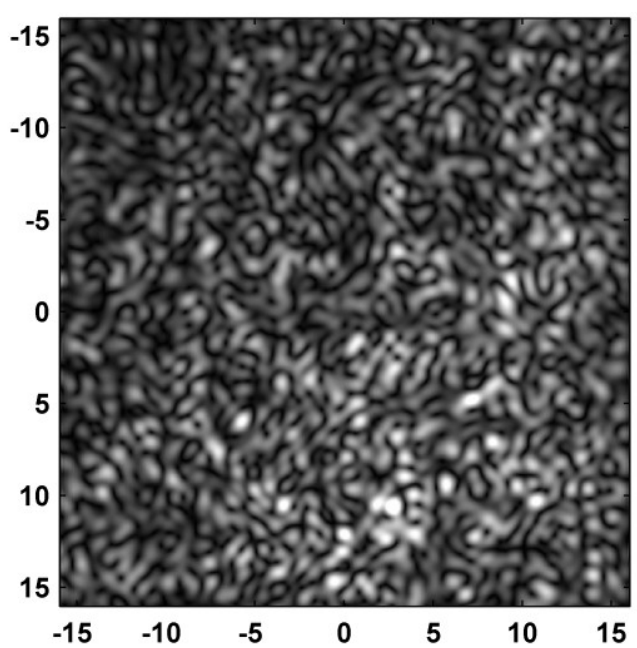

(a)

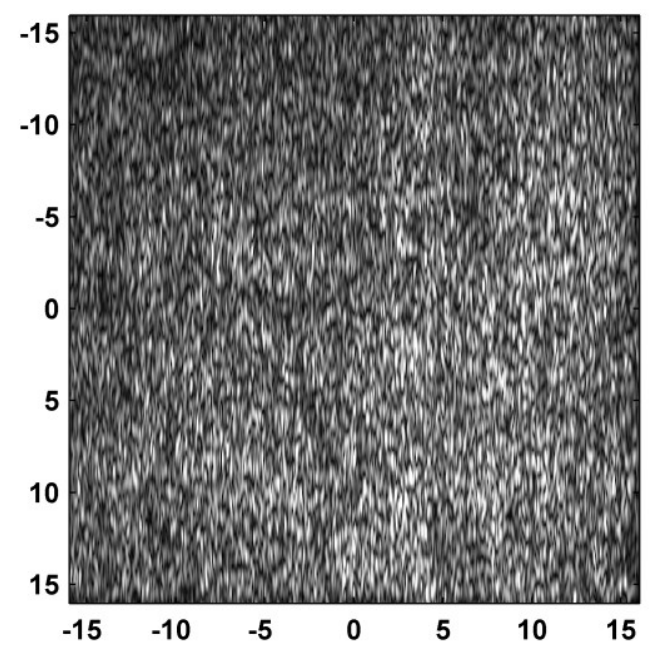

(b)

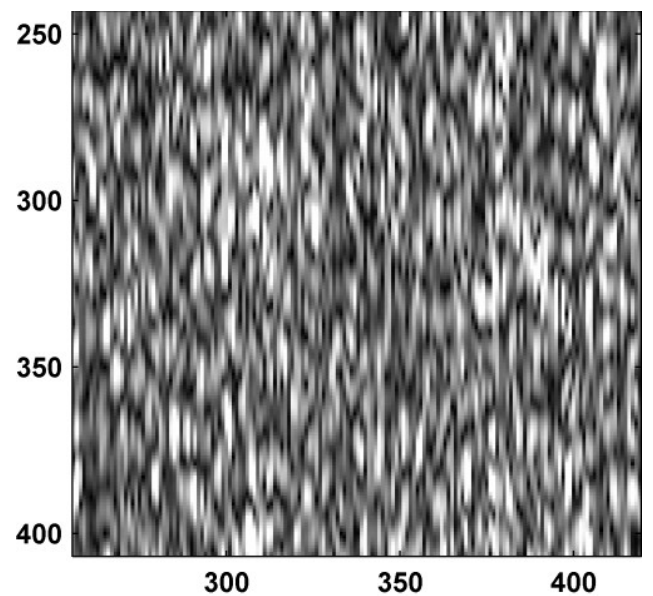

(c)

Figure 48: Side by side visual comparison of a, (a) $8 \mathrm{cyc} / \mathrm{mm}$ sub-aperture image and (b) HAL processed synthetic image and (c) a zoomed region of the HAL processed synthetic image. The vertical structure of the synthetic image is evident in the zoomed image.

\subsection{Quantitative Analysis}

Recall from the discussion following equation (17) that the theoretical diffraction limited spatial frequency bandwidth for single sub-aperture images is $1.38 \mathrm{cyc} / \mathrm{mm}$. As our real aperture is square, the theoretical cross-range MTF function is simply a straight line extending from unity at zero spatial frequency, to zero at $1.38 \mathrm{cyc} / \mathrm{mm}$ [19]. To compare the ideal theoretical single sub-aperture MTF to experimental results, four 
independent images of five Ronchi ruling targets, with spatial frequencies $0.25,0.5,0.75$, 1.0 and $1.25 \mathrm{cyc} / \mathrm{mm}$, were created and processed as described in the previous section. This allowed both mean MTF values and corresponding standard deviations to be calculated and plotted for each target frequency. The results are shown in Figure 49.

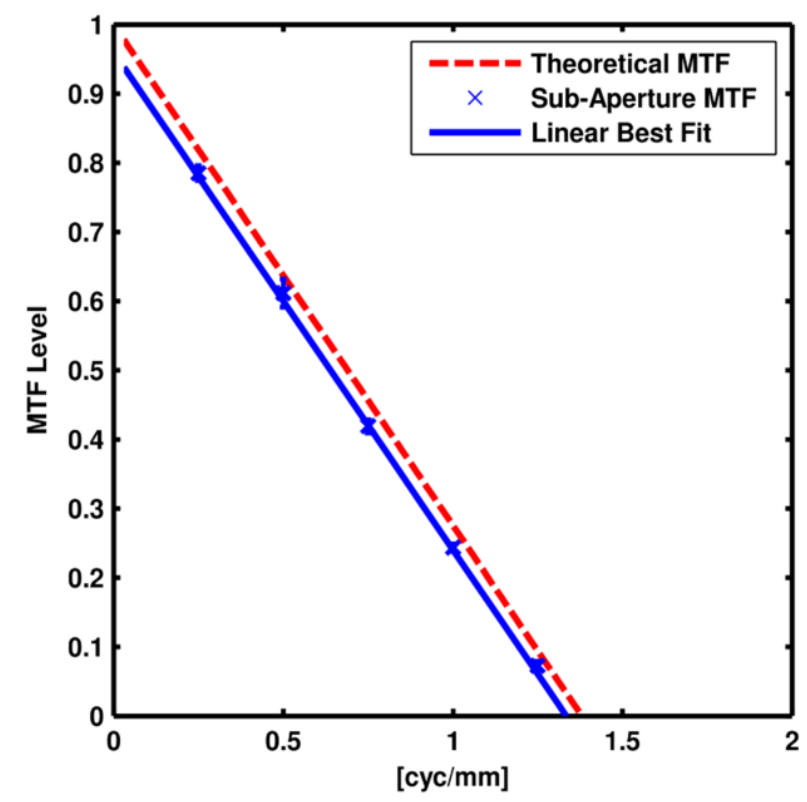

Figure 49: Theoretical and experimental MTF functions for single sub-aperture imaging.

The dashed line in Figure 48 demonstrates the theoretical single sub-aperture MTF, while the experimental mean and standard deviation values are shown by the $x$ 's and the vertical uncertainty bars, respectively. As demonstrated by the small uncertainties, we found our data to be quite repeatable for most spatial frequencies. Figure 48 also includes the linear best fit (i.e., the experimental MTF function) to the mean MTF data point values. The equation for this best fit line was found to be

$$
M T F_{\text {sub-aperture }}=0.96-0.72 f_{x} \text {, }
$$


where $f_{x}$ is a spatial frequency variable with units of cyc $/ \mathrm{mm}$. By setting $M T F_{\text {sub-aperture }}$ to zero in equation (34) and solving for $f_{x}$ we find the experimental single sub-aperture cutoff frequency to be $1.33 \mathrm{cyc} / \mathrm{mm}$. This is only $3.6 \%$ less than the theoretical, diffraction limited expected value.

Next, Figure 50 shows the theoretical and experimental MTFs for images formed after the full HAL processing of twelve overlapping, sequential collected, subaperture frames of data.

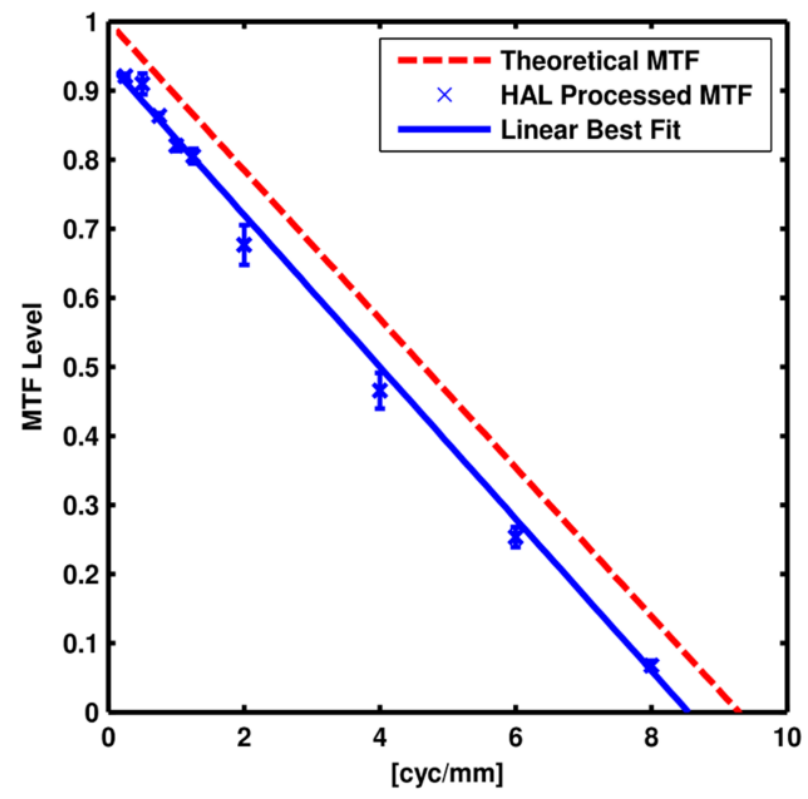

Figure 50: Theoretical and experimental MTF functions for synthetic images formed using 12 overlapping frames of fully HAL processed data.

The theoretical cut-off frequency in this case was found by multiplying the theoretical single sub-aperture cut-off frequency by the 12-shot HAL image sharpening ratio. Using equation (19) when $N=12$ yields an ISR of 6.73 , for a corresponding theoretical cut-off of $9.29 \mathrm{cyc} / \mathrm{mm}$. The results for the full HAL processed MTF can be easily viewed in Table 5. 


\begin{tabular}{lccc}
\hline \multicolumn{1}{c}{ Baseline Metrics } & Values and Results & & Percent Error \\
\cline { 1 - 1 } Theoretical HAL Cut-off & & $9.29 \mathrm{cyc} / \mathrm{mm}$ & \\
HAL Cut-off & $8.55 \mathrm{cyc} / \mathrm{mm}$ & $7.97 \%$ \\
Theoretical HAL ISR & 6.73 & \\
HAL ISR & 6.43 & & $4.46 \%$ \\
\hline
\end{tabular}

Table 5: A table constructed to show the metrics used to analyze the performance of the HAL processed MTF for $\mathrm{N}=12$ sub-shots. The system baseline values and results are shown as well as percent error between theoretical and actual measurements.

The experimental MTF was found by creating five independent images of a collection of Ronchi ruling targets with the following spatial frequencies: $0.25,0.5,0.75$, $1,1.25,2,4,6$ and $8 \mathrm{cyc} / \mathrm{mm}$. As stated before, these were created and processed as described in the Chapter 4. This once again allowed both mean MTF values and corresponding standard deviations to be calculated and plotted for each target frequency. Even when processing many frames of date, the small uncertainties we experienced demonstrate the robust and repeatable nature of our methods.

The linear best fit through the mean experimental MTF values plotted in Figure 50 was found to be,

$$
M T F_{H A L-12 s h o t}=0.94-0.11 f_{x} .
$$

This function yields an experimental cut-off frequency of $8.55 \mathrm{cyc} / \mathrm{mm}$, a value somewhat less than $8 \%$ below the theoretical expectation. In addition, by dividing the experimental 12-shot cutoff frequency by the experimental single sub-aperture cut-off frequency we find the experimental 12 -shot image sharpening ratio to be 6.43 . This is only $4.46 \%$ less than the corresponding theoretical maximum ISR. While this demonstrates the exceptional image sharpening potential of HAL processing methods, in practice the image sharpening ratio will ultimately be limited by accumulated phase errors and 
aberrations that are sure to arise when processing vast quantities of complex data. We should also note that in most cases we did not find that iteratively performing the registration steps outlined in Figure 30 noticeably improved our results.

Not surprisingly, we have found that more nearly diffraction limited performance can be realized if we are willing to sacrifice image sharpening by processing fewer subframes of data. For example, Figure 51 shows the theoretical and experimental MTF for images formed after processing only 8 sequential sub-aperture frames of the data previously collected.

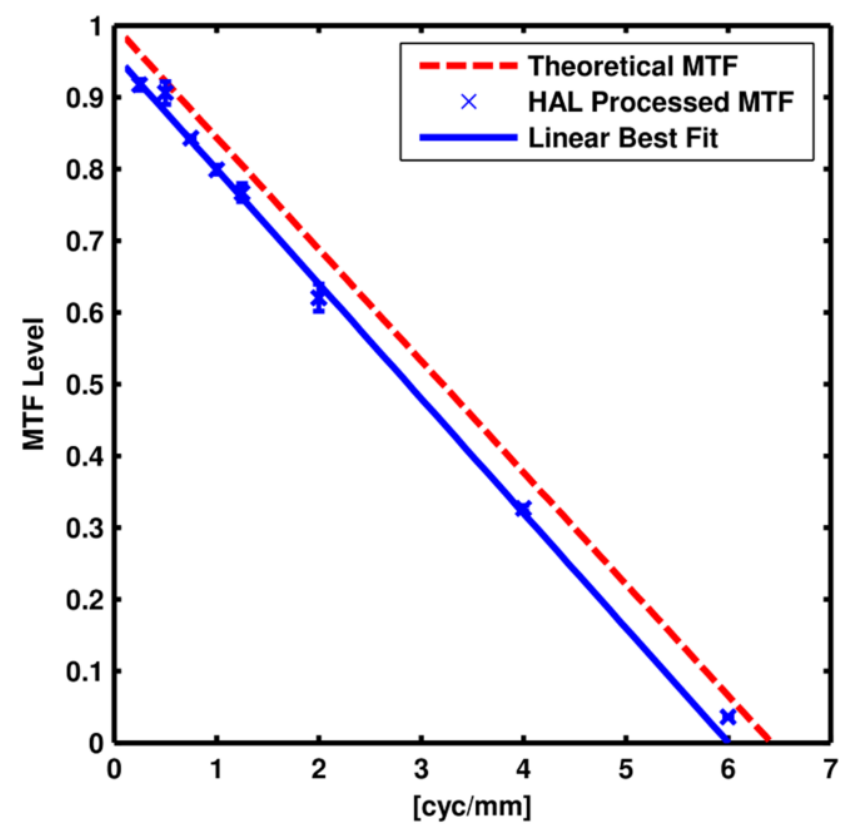

Figure 51: Theoretical and experimental MTF functions for synthetic images formed using 8 overlapping frames of fully HAL processed data. More nearly diffraction limited performance is realized in exchange for reduced image sharpening.

In this case, using equation (36) with $\mathrm{N}=8$, the 8 -shot ISR was found to be 4.65 , yielding a theoretical MTF cut-off frequency of $6.41 \mathrm{cyc} / \mathrm{mm}$. The associated linear best fit through the experimental mean MTF values shown in Figure 51 was in turn found to be,

$$
M T F_{H A L-8 s h o t}=0.96-0.16 f_{x} \text {. }
$$


In this case we found the experimental cut-off frequency to be $6.00 \mathrm{cyc} / \mathrm{mm}$. This is only $6.4 \%$ less than the theoretical expectation. Similarly the experimental image sharpening ratio is 4.5 , a value only $3.7 \%$ less than the theoretical maximum. The results for the 8 frame HAL MTF can also be viewed in Table 6.

\begin{tabular}{lcccc}
\multicolumn{1}{c}{ Baseline Metrics } & Values and Results & & Percent Error \\
\cline { 1 - 1 } Theoretical HAL Cut-off & & $6.41 \mathrm{cyc} / \mathrm{mm}$ & \\
HAL Cut-off & $6.00 \mathrm{cyc} / \mathrm{mm}$ & $6.40 \%$ \\
Theoretical HAL ISR & 4.65 & \\
HAL ISR & 4.51 & $3.01 \%$ \\
\hline
\end{tabular}

Table 6: A table constructed to show the metrics used to analyze the performance of the HAL processed MTF for $\mathrm{N}=8$ sub-shots. The system baseline values and results are shown as well as percent error between theoretical and actual measurements.

Figure 52 shows the HAL processed MTF plot containing $N=5$ sub-apertures. The effective aperture containing five overlapping sub-apertures will produce a theoretical ISR of 3.09. The linear best fit line through the average values was found to be,

$$
M T F_{H A L-5 \text { shot }}=-0.24 f_{x}+0.97 \text {. }
$$

The theoretical cut-off was found to be $4.26 \mathrm{cyc} / \mathrm{mm}$ while the HAL processed cut-off was found using Eq. (37) and solving for $f_{x}$ when the MTF is evaluated at zero. This value was found to be $4.04 \mathrm{cyc} / \mathrm{mm}$ with a percent error $5.16 \%$. The HAL ISR was found by the ratio of the experimental HAL cut-off and sub-aperture cut-off to be 3.04 with a percent error from theoretical of $1.6 \%$. The results for the 5 frame HAL processed MTF can also be viewed in Table 7 .

This figure shows almost diffraction limited performance of the HAL processed MTF. The small standard deviations of the error bar plot also indicate the repeatability of the HAL processing method. 


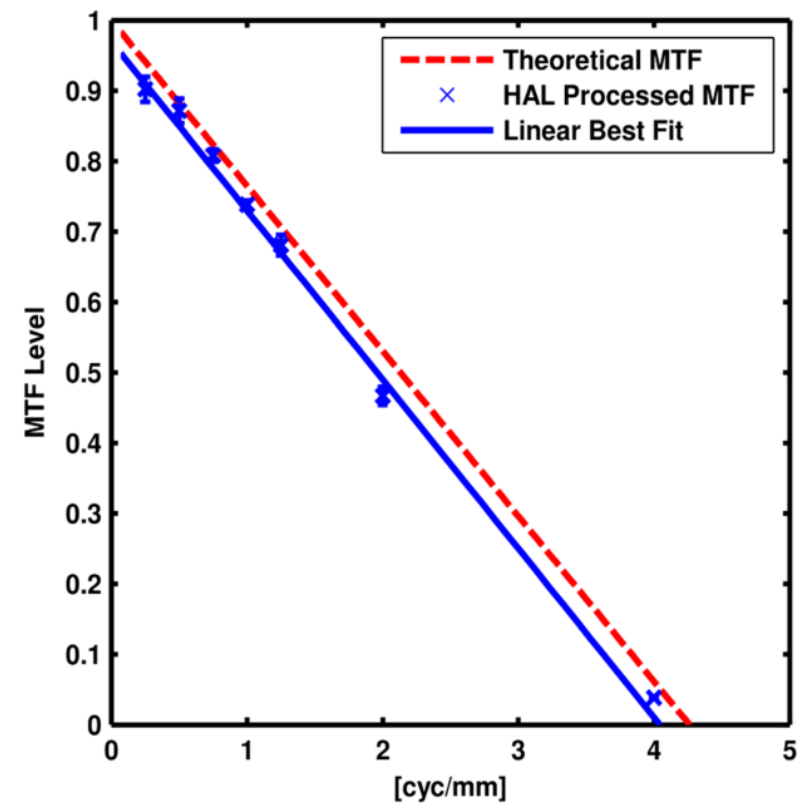

Figure 52: Theoretical and experimental MTF functions for synthetic images formed using 5 overlapping frames of fully HAL processed data. More nearly diffraction limited performance is realized in exchange for reduced image sharpening.

The results that are displayed in Table 7 show very close agreement to theoretical values that were calculated for the particular system.

\begin{tabular}{lcccc}
\hline \multicolumn{1}{c}{ Baseline Metrics } & & Values and Results & & Percent Error \\
\cline { 1 - 1 } Theoretical HAL Cut-off & & $4.26 \mathrm{cyc} / \mathrm{mm}$ & \\
HAL Cut-off & & & $5.16 \%$ \\
Theoretical HAL ISR & 3.09 & & \\
HAL ISR & 3.04 & $1.6 \%$ \\
\hline
\end{tabular}

Table 7: A table constructed to show the metrics used to analyze the performance of the HAL processed MTF for $\mathrm{N}=5$ sub-shots. The system baseline values and results are shown as well as percent error between theoretical and actual measurements.

For the results show below in Figure 53, both the sub-aperture MTF and the full $N=12$ HAL processed MTF are displayed. In this plot, the lines plotted through the data points are the best fit lines, which in turn represent the MTF. The overlay of both MTF lines makes it obviously clear how well the HAL processing algorithm has performed. 


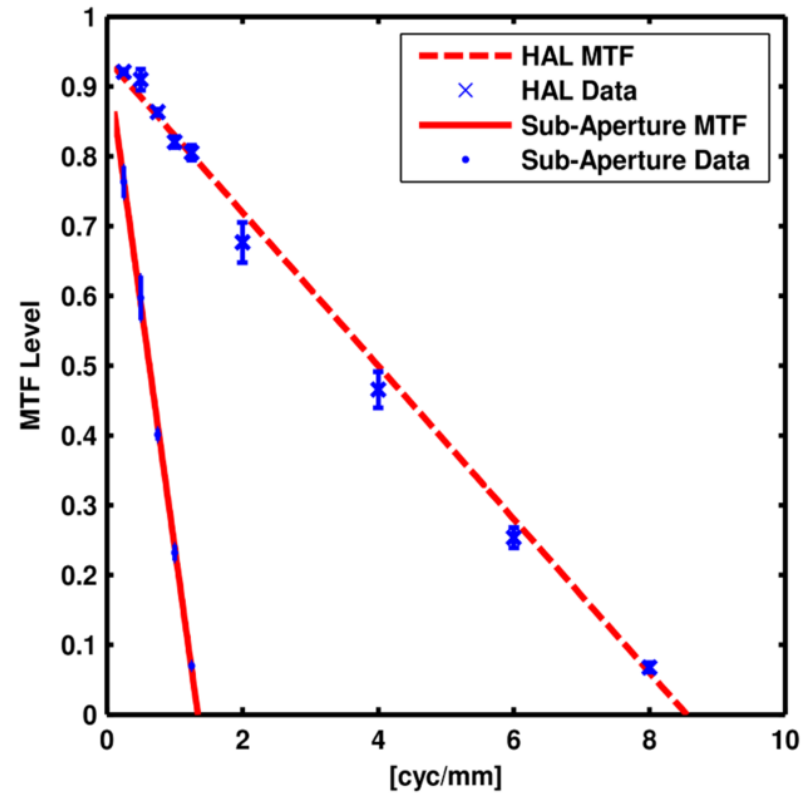

Figure 53: Experimental sub-aperture and full HAL processed MTF displayed on the same plot to show the cut-off gain 


\section{CHAPTER 7}

\section{CONCLUSIONS}

We have developed a HAL processing method based on the principles of the Stripmap HAL transform that accounts for phase errors across a synthetic aperture and provides quantitative proof, in the form of a modulation transfer function, that theoretical predictions of the HAL ISR can be achieved. After the sub-aperture segments are collected, the post processing techniques detailed in the HAL processing method are extremely time efficient taking only a matter of seconds. Holographic Aperture Ladar (HAL) is a variation of synthetic aperture ladar that uses a translating two dimensional imaging sensor rather than a single point detector. While the theory that formed the HAL transform has been verified for a point target, the information presenting in this paper shows HAL's ability to account for phase errors that are accumulated over a synthetic aperture.

\subsection{Original Contributions}

For the research presented in this paper, multiple sub-aperture pupil frames were

collected at locations approximately $D_{a p} / 4$ to ensure that neighboring pupil field segments overlapped. In the laboratory experiment, the principle of relative motion was use where the target was moved to different location perpendicular to the illumination beam while the transmitter, receiver and local oscillator were held fixed. A $H A L$ 
Processing Method was then developed to process the sub-aperture field segments. The overlapping segments were then cropped where there were like areas and speckled correlated; applying the corrections to the Fourier transform of the full pupil field as a linear phase tilt. After an inverse Fourier transform the field segments would properly shift to sub-pixel accuracy. The second registration step involved registering complex image plane segments; again where neighboring frames overlap. The corrections are then applied as a linear phase tilt to the full pupil fields to develop the proper phase bowl for the synthetic aperture. The last phase correction of piston phase error was corrected and the synthetic pupil can then be formed. After an amplitude equalization over the areas of overlap in the synthetic pupil, a Fourier transform is then applied to gain access to the higher resolution synthetic image. The target image is then cropped out, inverse Fourier transformed, zero-padded and Fourier transformed back to the image plane to produce an up-sampled image. The complex value image is then converted to an intensity image, zero-padded to provide enough samples under the spatial frequency harmonics and then Fourier transformed to view the harmonics. The first harmonic was the term of interest and the maximum value was located, normalized to the $\mathrm{DC}$ peak value and then the $\mathrm{DC}$ scaling factor was applied. This value was plotted on the HAL Processed MTF graph. The process was repeated for multiple targets that enveloped the effective aperture dictated by theoretical calculations.

\subsection{Future Work}

Future work will involve the analysis of the effect of signal power on the HAL processing method. Measurements will be made to show how the reduction of transmitter 
signal power will affect the registration algorithm. The expansion of the HAL processing algorithm from using the side looking, Stripmap HAL transformation to the Spotlight HAL transformation would also be a valuable technique to approach. 


\section{REFERENCES}

[1] S.M. Beck, J.R. Buck, W.F. Buell, R.P. Dickinson, D.A. Kozlowski, N.J. Marechal, and T.J. Wright, "Synthetic-Aperture Imaging Laser Radar: Laboratory Demonstration and Signal Processing,” Applied Optics, Vol. 44, No. 35, pp. 7621-7629 (2005)

[2] B.D. Duncan and M.P. Dierking; "Holographic aperture ladar”, Applied Optics, Vol. 48, Issue 6, pp. 1168-1177 (2009)

[3] J.W. Stafford, B.D. Duncan and M.P. Dierking; "Experimental demonstration of a stripmap holographic aperture ladar system”, Applied Optics, Vol. 49, Issue 12, pp. $2262-2270(2010)$

[4] M.A. Richards, “Fundamentals of Radar Signal Processing”, Chapter 8, McGrawHill, New York, NY (2005)

[5] M. Soumekh, "Synthetic Aperture Radar Signal Processing”, John Wiley \& Sons, New York, NY, (1999)

[6] N. Levanon and E. Mozeson, “Radar Signals”, John Wiley \& Sons, Hoboken, NJ, (2004) 
[7] D. Park; "Performance Analysis of Optical Synthetic Aperture Radars", Proc. of SPIE Vol. 999, Laser Radar III, pp. 100-116, (1988)

[8] T.G. Kyle; "High Resolution Laser Imaging System”, Applied Optics, Vol. 28, No. 13, pp. 2651-2656 (1989)

[9] T.J. Green, Jr., S. Marcus and B.D. Colella; "Synthetic-Aperture-Radar Imaging with a Solid-State Laser", Applied Optics, Vol. 34, No. 30, pp. 6941-6949 (1995)

[10] J.C. Marron and R.L. Kendrick; "Distributed Aperture Active Imaging”, Proc. of SPIE Vol. 6550, Laser Radar Technology and Applications XII, edited by M.D. Turner and G.W. Kamerman, paper 65500A (2007)

[11] R. Binet, J. Colineau and J.-C. Lehureau; "Short-Range Synthetic Aperture Imaging at $633 \mathrm{~nm}$ by Digital Holography", Applied Optics, Vol. 41, No. 23, pp. 4775-4782 (2002)

[12] J.-C. Lehureau and J. Colineau; "Optical Synthetic Aperture Imagery", Proc. of SPIE Vol. 5816, Optical Pattern Recognition XVI, edited by D.P. Casasent and T.-H. Chao, pp. 54-65, (2005)

[13] T.M. Kreis, M. Adams and W.P.O. Jueptner; "Aperture Synthesis in Digital Holography", Proc. of SPIE Vol. 4777, Interferometery XI: Techniques and Analysis, edited by K. Creath and J. Schmit, pp. 69-76 (2002)

[14] J.C. Marron and K.S. Schroeder; "Holographic Laser Radar", Optics Letters, Vol. 18, No. 5, pp. 385-387 (1993) 
[15] J.C. Marron, R.L. Kendrick, T.A. Hoft and N. Seldomridge; "Novel Multi-Aperture $3 D$ Imaging Systems", $14^{\text {th }}$ Coherent Laser Radar Conference, Snowmass, CO (2007)

[16] J.E. Mason, K.A. Anderson, R.L. Kendrick, T.S. Kubo, J.C. Maron and T. Zhao; "Experiments with Multi-Aperture Three-Dimensional Coherent Imaging", $14^{\text {th }}$ Coherent Laser Radar Conference, Snowmass, CO (2007)

[17] J.H. Shapiro; "Heterodyne mixing efficiency for detector arrays", Applied Optics, Vol. 26, Issue 17, pp. 3600-3606 (1987)

[18] A. Stern, B. Javidi; "General Sampling Theorem and Application in Digital Holography", Invited Paper, Optical Information Systems II, Proceedings of SPIE, Vol. 5557, pp. 110-123, edited by B. Javidi, D. Psaltis, SPIE, Bellingham, WA (2004)

[19] J.W. Goodman, "Introduction to Fourier Optics", $3^{\text {rd }}$ Ed., Chapters 3,4 and 6, Roberts \& Co., Englewood, CO (2005)

[20] A.E. Siegman, "Lasers", Chapter 17, University Science Books, Mill Valley, CA (1986)

[21] F.G. Stremler, "Introduction to Communication Systems", $3^{\text {rd }}$ Ed., Chapter 3, Addison-Wesley (1990)

[22] M. Guizar-Sicairos, S.T. Thurman and J.R. Fienup; “Efficient subpixel image registration algorithms", Optics Letters, Vol. 33, Issue 2, pp. 156-158 (2008) 
[23] Guizar, Manuel. "Efficient Subpixel Image Registration by Cross-Correlation." Matlab Central. MathWorks, Inc. (1994-2011)

$<$ http://www.mathworks.com/matlabcentral/fileexchange/18401-efficient-subpixelimage-registration-by-cross-correlation>

[24] J. R. Fienup, and J. J. Miller, "Aberration correction by maximizing generalized sharpness metrics,” J. Opt. Soc. Am. A 20(4), 609-619 (2003)

[25] D. J. Rabb, D. F. Jameson, J. W. Stafford and A. J. Stokes; “Distributed Aperture Synthesis", Optics Express, Vol. 18 Issue 10, pp.10334-10342 (2010)

[26] S. T. Thurman, and J. R. Fienup, "Phase-error correction in digital holography," J. Opt. Soc. Am. A 25(4), 983-994 (2008)

[27] A.E. Tippie and J.R. Fienup; "Multiple-plane anisoplanatic phase correction in a laboratory digital holography experiment”, Optics Letters, Vol. 35, Issue 19, pp. 32913293 (2010) 


\section{APPENDIX A}

\section{HAL DATA PROCESSING IN MATLAB}

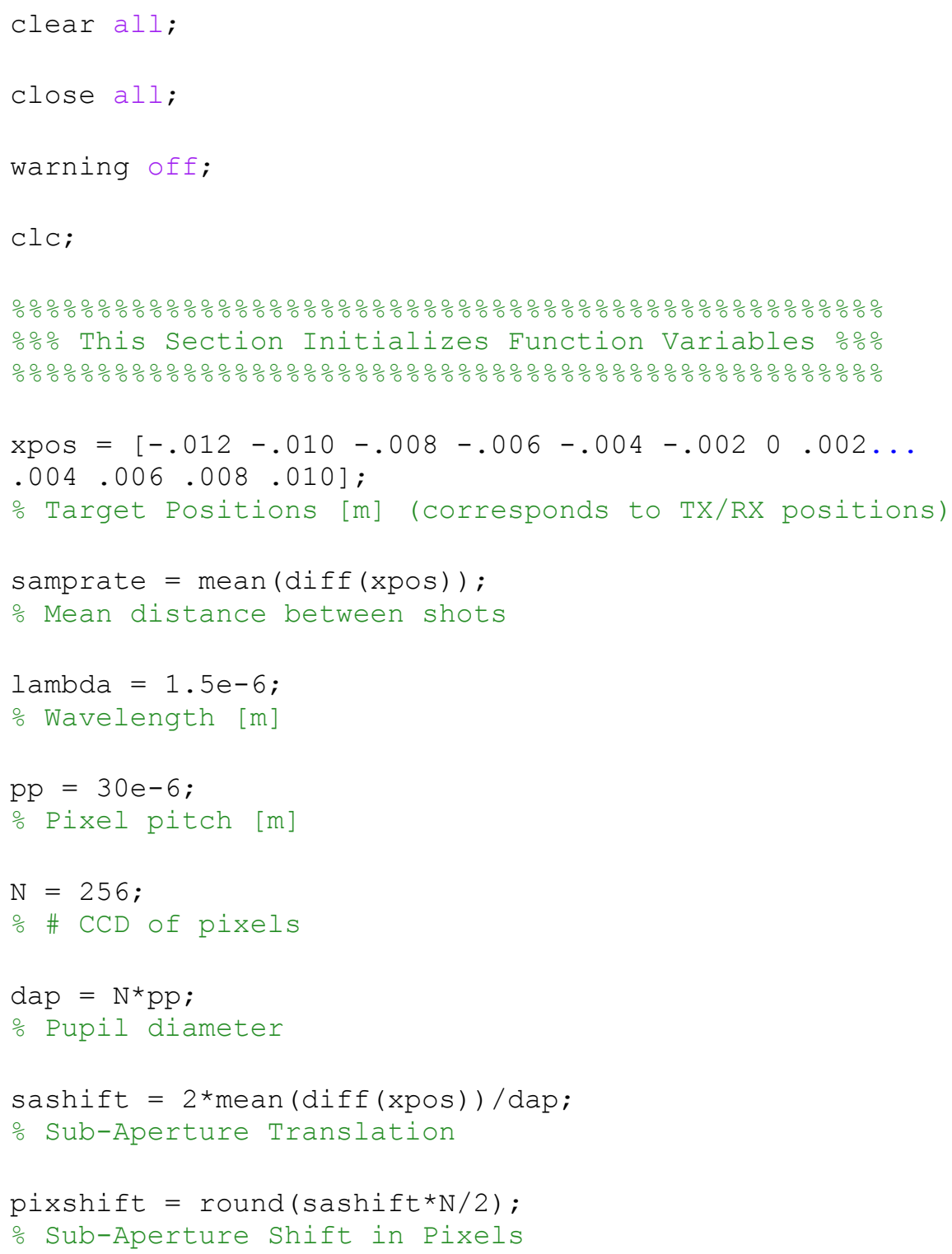




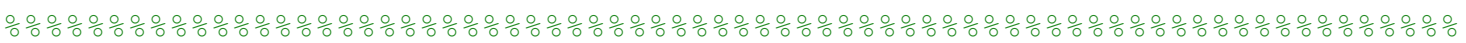
응응 This Section Loads the Sub-Apertures into Pre-Allocated Arrays 응 $\%$

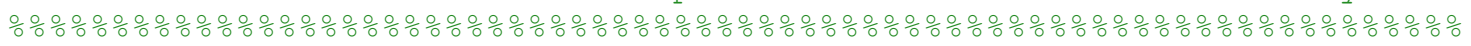

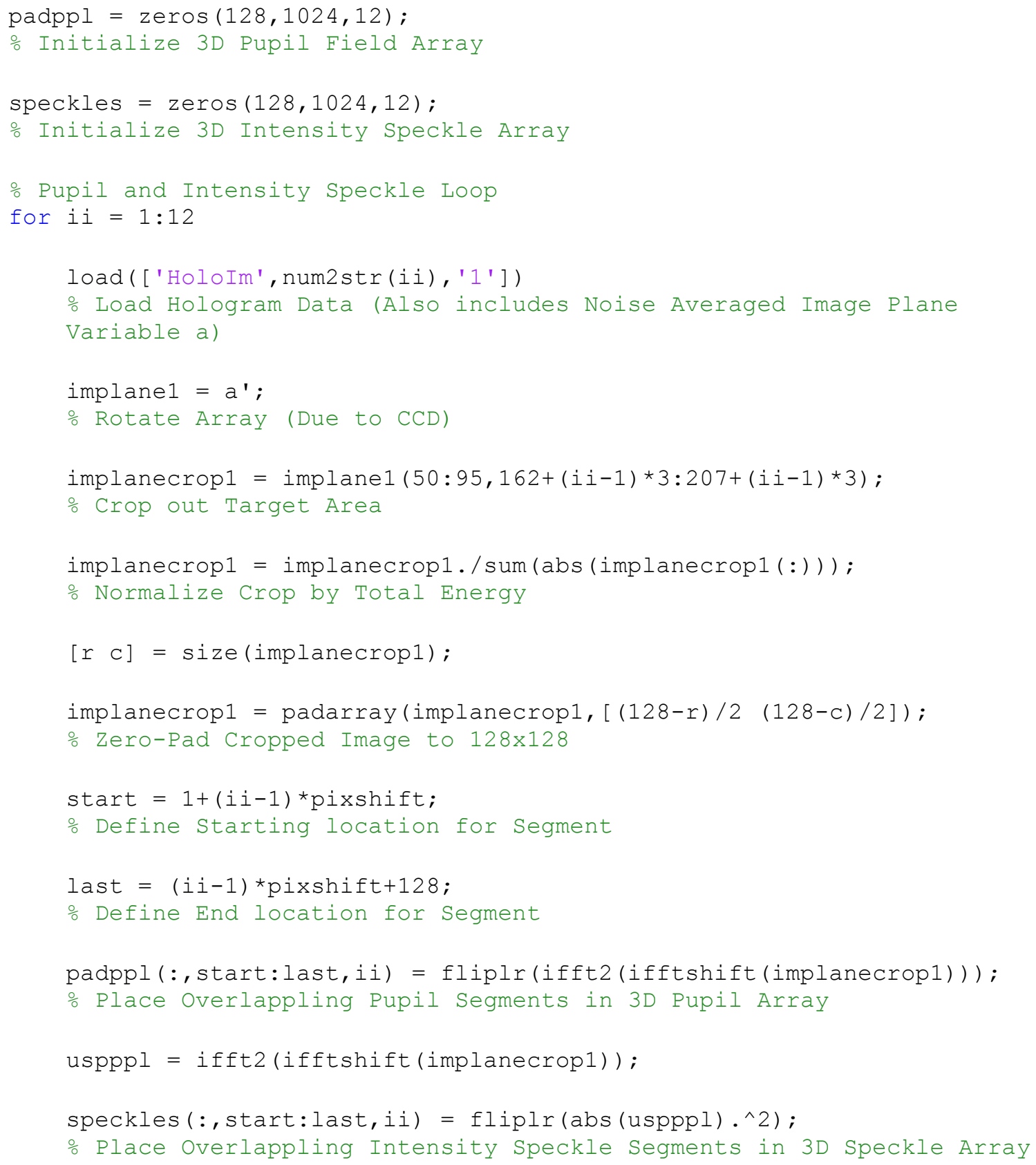




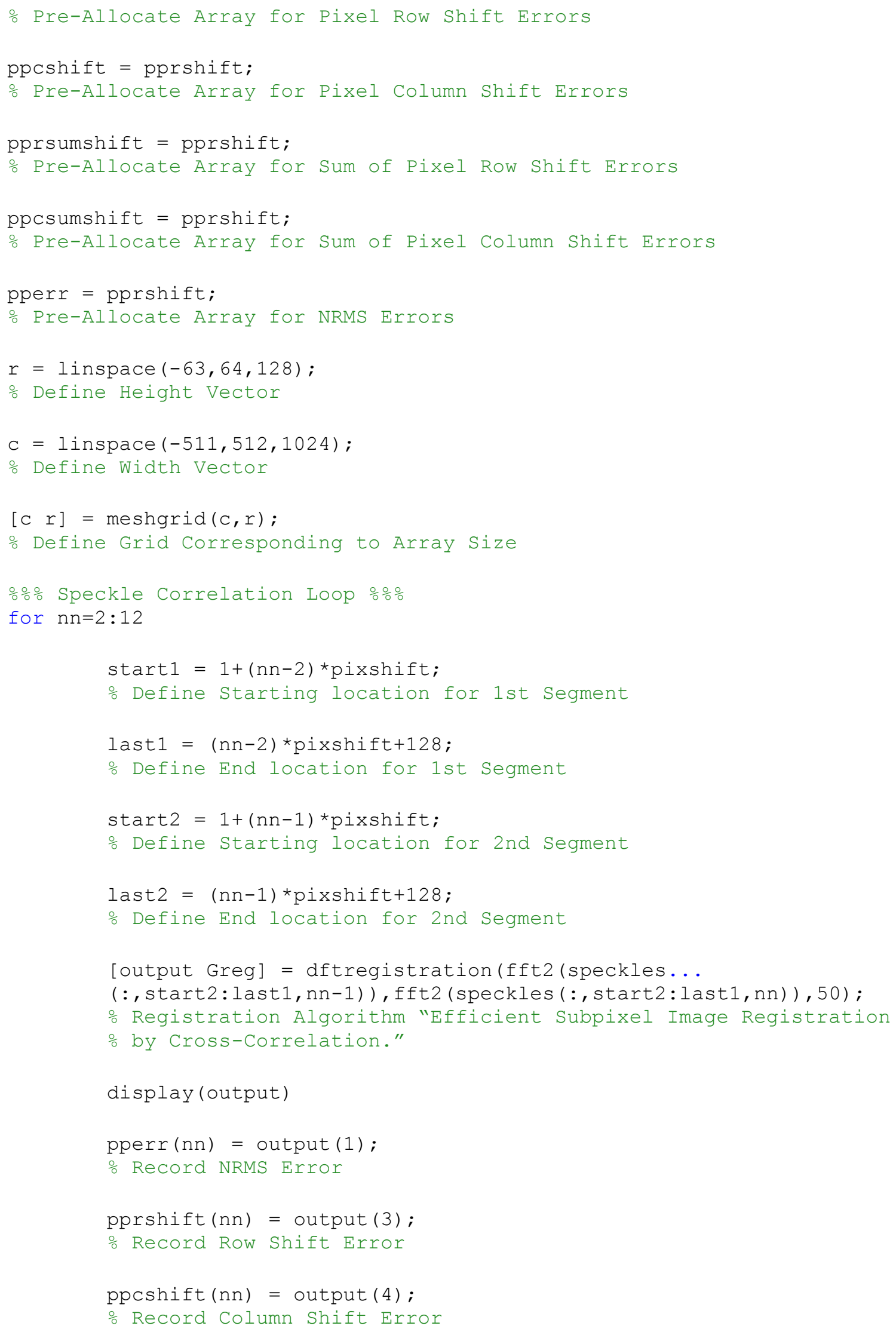




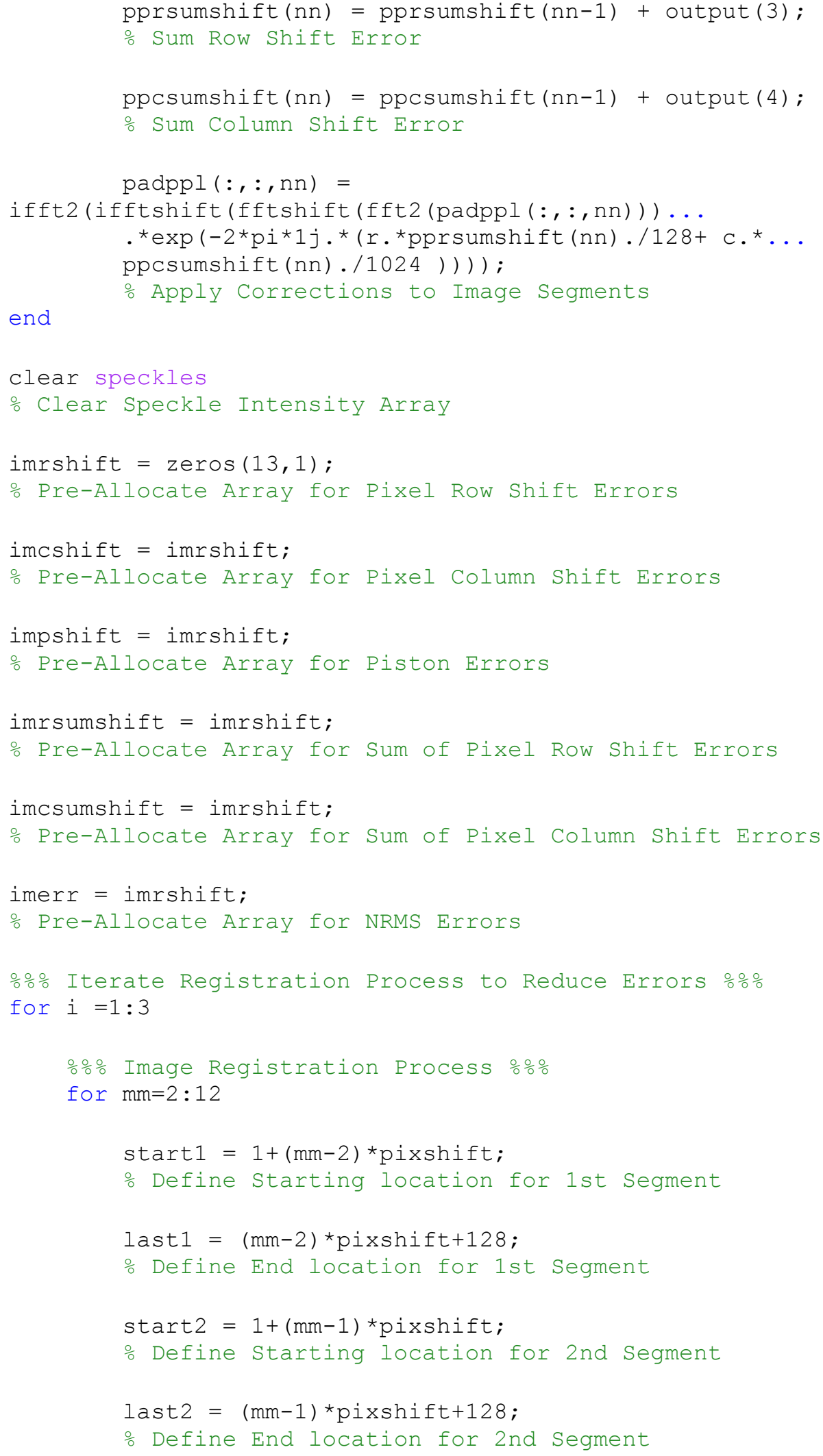




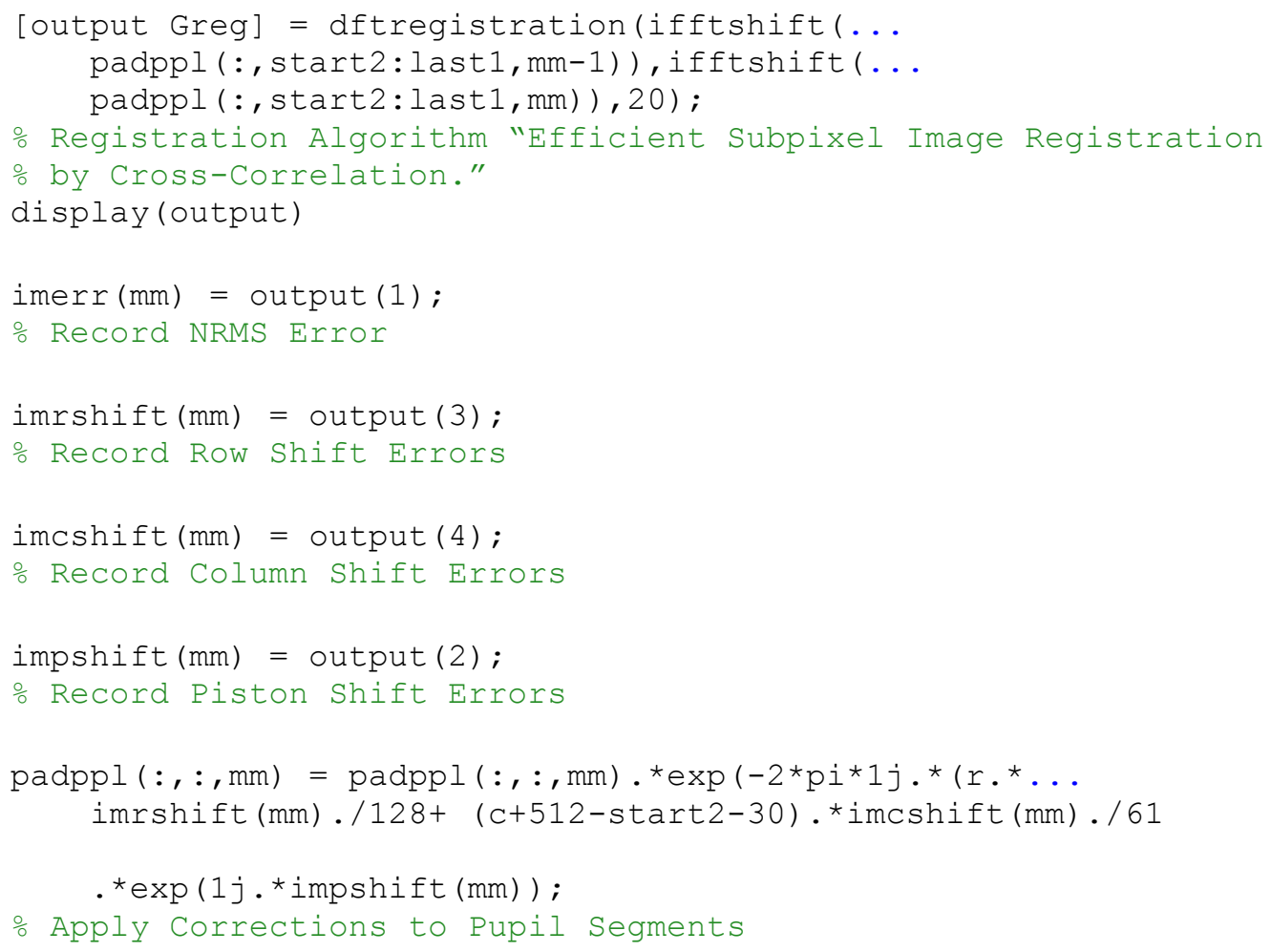




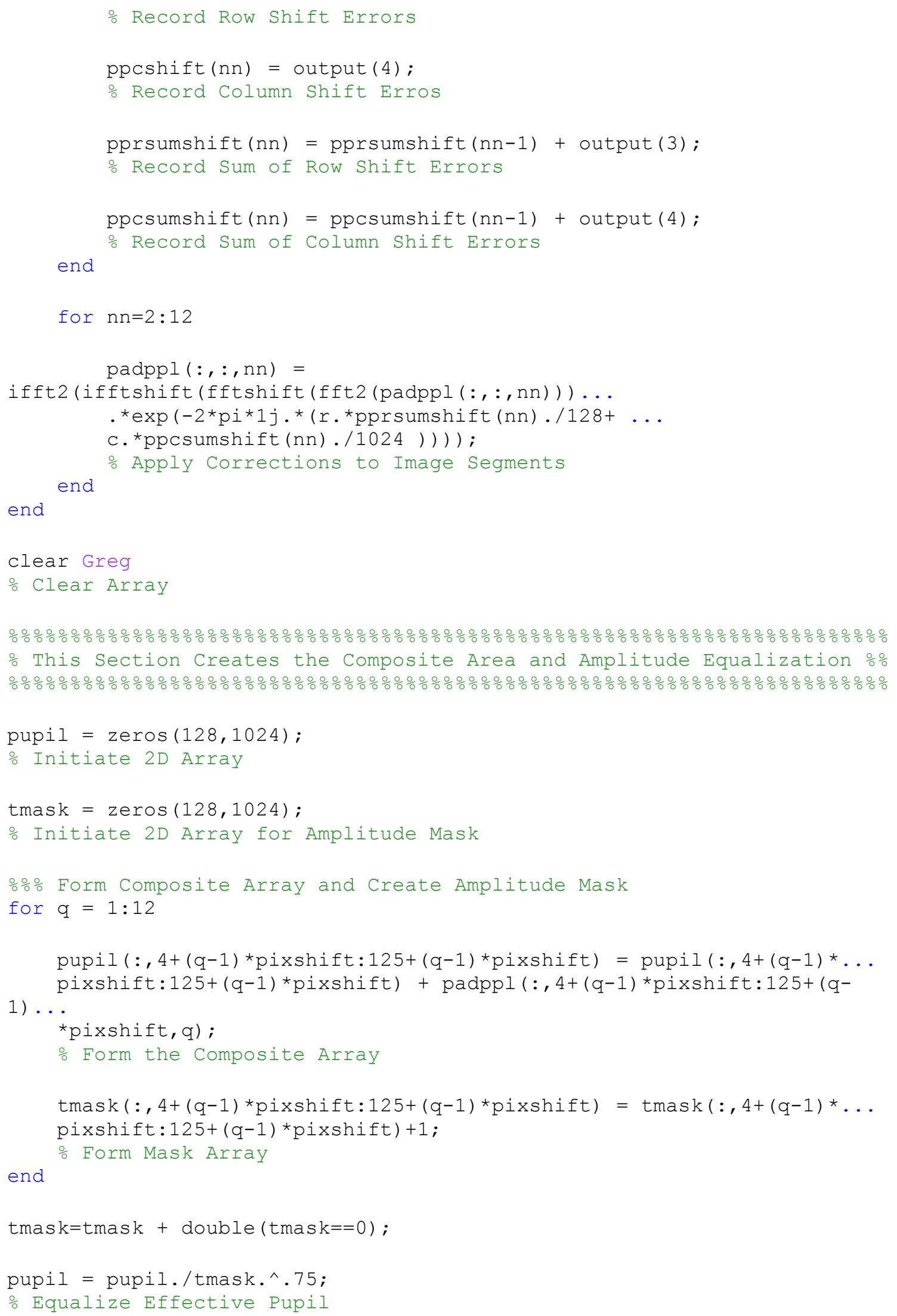




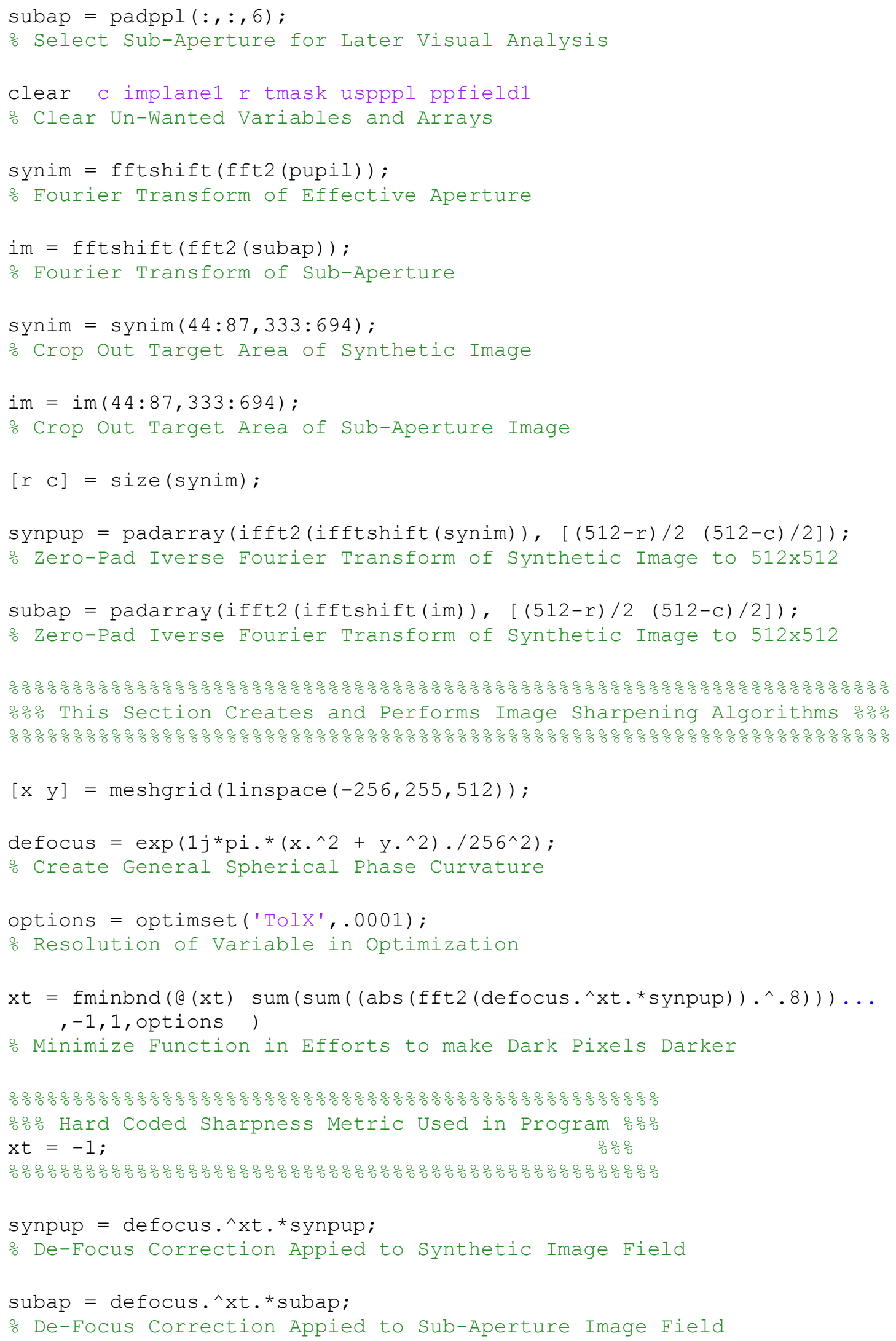




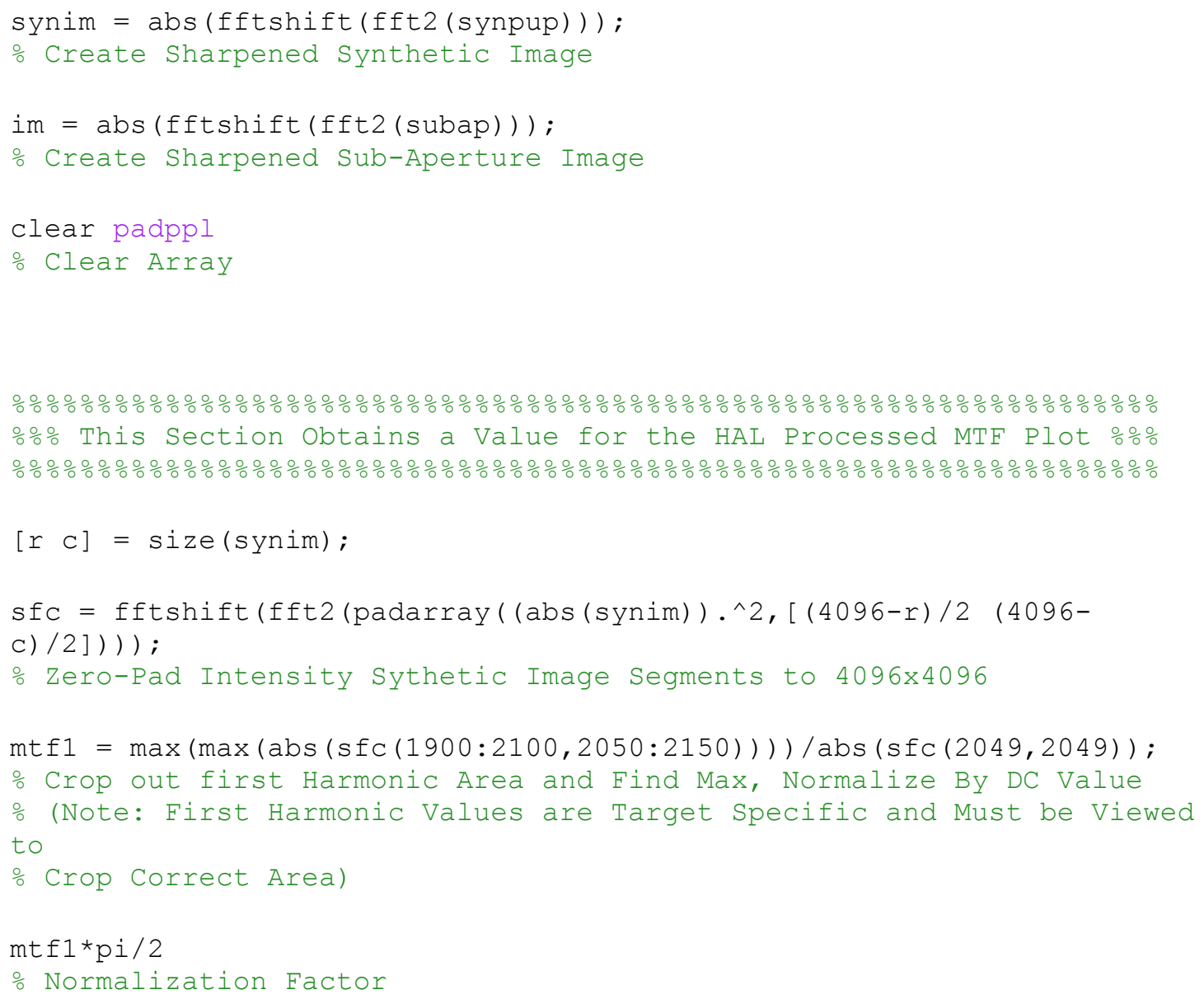




\section{APPENDIX B}

\section{DIGITAL HOLOGRAPHY}

In efforts to obtain and record target field information in the pupil plane, a CCD camera array will receive an intensity image of fringes. This recorded information is mixing of the modulus squared of the target information and the local oscillator fields. The intensity $I_{C C D}(x, y)$ on the CCD can be display implicitly as,

$$
\begin{gathered}
I_{C C D}(x, y)=\left|g_{t}(x, y)+f_{L O}(x, y)\right|^{2} \\
=\left|g_{t}(x, y)\right|^{2}+\left|f_{L O}(x, y)\right|^{2}+g_{t}(x, y) f_{L O}^{*}(x, y)+g_{t}^{*}(x, y) f_{L O}(x, y)
\end{gathered}
$$

where $g_{t}(x, y)$ and $f_{L O}(x, y)$ are the target and local oscillator fields respectively and the operator $*$ denotes the complex conjugate. For a distant target object, a Fourier transform of equation (B.1) will recover the target object field. This result can be seen as,

$$
\begin{aligned}
& \mathfrak{J}\left\{I_{C C D}(x, y)\right\}=\mathfrak{I}\left\{\left|g_{t}(x, y)\right|^{2}\right\}+\mathfrak{I}\left\{\left|f_{L O}(x, y)\right|^{2}\right\} \\
& +\mathfrak{J}\left\{g_{t}(x, y) f_{L O}^{*}(x, y)\right\}+\mathfrak{I}\left\{g_{t}^{*}(x, y) f_{L O}(x, y)\right\}
\end{aligned}
$$

The first two terms of equation (B.2) are the target and $\mathrm{LO}$ autocorrelation terms respectively. Assuming a uniform amplitude plane wave local oscillator that is just slightly tilted with respect to the optical axis by $\theta_{x}$ and $\theta_{y}$ will result in equation (B.3), 


$$
\begin{aligned}
& \mathfrak{I}\left\{I_{C C D}(x, y)\right\}=\mathfrak{I}\left\{\left|g_{t}(x, y)\right|^{2}\right\}+A_{L O}^{2} \delta\left(f_{x}, f_{y}\right) \\
& +\mathfrak{J}\left\{g_{t}(x, y) A_{L O} \exp \left(j k\left(\theta_{x} x+\theta_{y} y\right)\right)\right\} \\
& +\mathfrak{I}\left\{g_{t}^{*}(x, y) A_{L O} \exp \left(-j k\left(\theta_{x} x+\theta_{y} y\right)\right)\right\}
\end{aligned}
$$

where $k$ is the wave-vector number and $\delta$ is the Dirac-delta function. The result then follows as,

$$
\begin{aligned}
& \mathfrak{I}\left\{I_{C C D}(x, y)\right\}=\mathfrak{I}\left\{\left|g_{t}(x, y)\right|^{2}\right\}+A_{L O}^{2} \delta\left(f_{x}, f_{y}\right) \\
& +A_{L O} \mathfrak{I}\left\{g_{t}(x, y)\right\} \otimes \delta\left(f_{x}-f_{x o}, f_{y}-f_{y o}\right) \\
& +A_{L O} \mathfrak{I}\left\{g_{t}^{*}(x, y)\right\} \otimes \delta\left(f_{x}+f_{x o}, f_{y}+f_{y o}\right)
\end{aligned}
$$

where the offset frequencies $f_{x o}$ and $f_{y o}$ are resulted from the tilt angles $\theta_{x}$ and $\theta_{y}$ applied to the local oscillator and the operator $\otimes$ represents the convolution process. The tilted local oscillator will therefore shift the target image as well as the conjugate image in the Fourier plane away from the central autocorrelation terms. The third term of equation (B.4) will then contain the complex target information along with local oscillator information. 


\section{APPENDIX C}

\section{OSA PERMISSION}

\footnotetext{
${ }^{i}$ OSA considers your requested use of its copyrighted material to be Fair Use under United States Copyright Law. It is requested that a complete citation of the original material be included in any publication. In this case, we are waiving any applicable permissions fees, and the figures may be reproduced free of charge.
} 